REVIEWS OF MODERN PHYSICS, VOLUME 82, APRIL-JUNE 2010

\title{
Long range interactions in nanoscale science
}

Roger H. French*

DuPont Co. Central Research, E400-5207 Experimental Station, Wilmington, Delaware 19880, USA

and Department of Materials Science and Engineering, University of Pennsylvania,

Philadelphia, Pennsylvania 19104, USA

V. Adrian Parsegian ${ }^{\dagger}$

Laboratory of Physical and Structural Biology, NICHD, National Institutes of Health, Bethesda, Maryland 20892-0924, USA

Rudolf Podgornik

Laboratory of Physical and Structural Biology, NICHD, National Institutes of Health, Bethesda, Maryland 20892-0924, USA;

Faculty of Mathematics and Physics, University of Ljubljana, Ljubljana SI-1000, Slovenia; and Department of Theoretical Physics, J. Stefan Institute, Ljubljana 1000, Slovenia

Rick F. Rajter

Department of Materials Science and Engineering, Massachusetts Institute of Technology, Cambridge, Massachusetts 02139-4307, USA

Anand Jagota

Department of Chemical Engineering and Bioengineering Program, Lehigh University, Bethlehem, Pennsylvania 18015, USA

Jian Luo

School of Materials Science and Engineering, Clemson University, Clemson, South Carolina 29634, USA

Dilip Asthagiri

Department of Chemical and Biomolecular Engineering, Johns Hopkins University, Baltimore, Maryland 21218, USA

Manoj K. Chaudhury

Department of Chemical Engineering, Lehigh University, Bethlehem, Pennsylvania 18015, USA

Yet-ming Chiang

Department of Materials Science and Engineering, Massachusetts Institute of Technology, Cambridge, Massachusetts 02139, USA

Steve Granick

Materials Research Laboratory, University of Illinois, Urbana, Illinois 61801, USA

Sergei Kalinin

Materials Science and Technology Division and The Center for Nanophase Materials

Science, Oak Ridge National Laboratory, Oak Ridge, Tennessee 37831, USA

Mehran Kardar

Department of Physics, Massachusetts Institute of Technology, Cambridge, Massachusetts 02139, USA

Roland Kjellander

Department of Chemistry, University of Gothenburg, SE-412 96 Gothenburg, Sweden

David C. Langreth

Center for Materials Theory, Department of Physics and Astronomy, Rutgers University,

Piscataway, New Jersey 08854-8019, USA 


\section{Jennifer Lewis}

Frederick Seitz Materials Research Laboratory, Materials Science and Engineering Department, University of Illinois, Urbana, Illinois 61801, USA

\section{Steve Lustig}

DuPont Co. Central Research, E400-5472 Experimental Station, Wilmington, Delaware 19880, USA

\section{David Wesolowski}

Chemical Sciences Division, Oak Ridge National Laboratory, P.O. Box 2008, Oak Ridge, Tennessee 37831-6110, USA

\section{John S. Wettlaufer}

Department of Geology and Geophysics, Department of Physics, Program in Applied Mathematics, Yale University, New Haven, Connecticut 06520-8109, USA

\section{Wai-Yim Ching}

Department of Physics, University of Missouri-Kansas City, Kansas City, Missouri 64110, USA

\section{Mike Finnis}

Department of Materials and Department of Physics, Imperial College London, Exhibition Road, London SW7 2AZ, United Kingdom

\section{Frank Houlihan}

AZ Electronic Materials Corporation USA, 70 Meister Avenue, Somerville, New Jersey 08876, USA

\section{O. Anatole von Lilienfeld}

Multiscale Dynamic Material Modeling Department, Sandia National Laboratories, Albuquerque, New Mexico 87185, USA

\section{Carel Jan van Oss}

Department of Microbiology and Immunology, Department of Chemical and Biological Engineering, and Department of Geology, State University of New York at Buffalo, Buffalo, New York 14260, USA

\section{Thomas Zemb}

Institut de Chimie Séparative de Marcoule, UMR 5257, 30207 Bagnols sur Cèze, France

(Published 11 June 2010)

Our understanding of the "long range" electrodynamic, electrostatic, and polar interactions that dominate the organization of small objects at separations beyond an interatomic bond length is reviewed. From this basic-forces perspective, a large number of systems are described from which one can learn about these organizing forces and how to modulate them. The many practical systems that harness these nanoscale forces are then surveyed. The survey reveals not only the promise of new devices and materials, but also the possibility of designing them more effectively.

*Corresponding author. rogerhfrench@longrangeinteractions.com

${ }^{\dagger}$ Present address: Department of Physics, University of Massachusetts, Amherst, MA 01003, USA. 


\section{CONTENTS}

I. Introduction

II. Fundamental Interactions

A. Electrodynamic interactions

1. Lifshitz theory from optical properties

a. Hamaker coefficients

b. Full spectral optical properties

c. Model systems: PS-water-PS and $\mathrm{SiO}_{2}$-water- $\mathrm{SiO}_{2}$

d. Advanced effects and phenomenology

2. Ab initio optical properties of complex materials

3. Electrodynamic interactions for arbitrary shapes

a. Cylinders and plates

b. Spheres and compact shapes

4. Noncovalent interactions from electronic structure calculations

a. Recently evolving density functional theory methods

i. Many-body methods using Kohn-Sham orbitals

ii. Explicit density functional methods

b. $C_{6}$ coefficients of polarizabilities, and damping functions

5. Challenges and opportunities

B. Electrostatic interactions

1. Electrostatics in equilibrium statistical mechanics: Electrostatic double layers

a. Model system: DNA-DNA interactions in charged electrolytes

b. Electrostatics with surfaces and interfaces

2. Electrostatics in density functional theory

3. Challenges and opportunities

C. Polar interactions

1. Motivation and recent advances

2. Challenges and opportunities

III. Instructive Systems

A. Atoms and molecules

1. Optical spectra and Lifshitz theory for complex biomolecular systems

a. Optical properties of SWCNTs

b. Optical properties of B-DNA

2. Hydration interaction and ionic specificity

3. Extraction, separation, and phase transfer reactions

4. DFT results on DNA base-pair vdW interactions

B. Interfaces, surfaces, and defects in solids

1. Impurity-based quasiliquid surficial and interfacial films

2. Charged defects in solids

C. Solid/liquid interfaces and suspensions

1. Water and ice

a. The phase architecture of ice

b. Optical properties of ice and water

2. Hydration

3. Structure and dynamics at oxide/electrolyte
1889

1890

1891

1892

1892

1893

1893

1894

1895

1895

1896

1897

1897

1898

1898

1898

1899

1900

1900

1901

1901

1902

1902

1903

1904

1904

1905

1906

1906

1906

1907

1907

1908

1908

1909

1909

1909

1910

1911

1911

1911

1912

1912 interfaces

4. Colloidal suspensions

5. Solution-based manipulation of SWCNT

a. Dispersion and structure

b. Sorting and placement

IV. Harnessing LRIs

A. Surfaces and interfaces

1. Proton exchange membranes for hydrogen fuel cells

1913

2. Intergranular and surficial films

3. Premelting dynamics and its manipulation

B. Colloids and self-assembly

1. Tailored building blocks: From hard spheres to patchy colloids

1916

1917

1918

1918

1919

1919

1920

1922

1923

1925

2. Synthesis and assembly of designer colloidal building blocks

3. Challenges and opportunities

1925

1925

1926

C. Self-assembly and emerging device applications

1927

1. Electrochemical devices: Li-ion batteries from heterogeneous colloids

2. Active electronic devices: Single-walled carbon nanotubes

1927

D. Nanoscale probes of long range interactions 1929

1. Scanning probes

a. The SPM approach

i. Direct force measurements

ii. Voltage modulation approaches

iii. Functional probes

iv. Probing dissipative dynamics

b. Future developments

1929

1929

1930

1930

1931

1931

1931

2. Scattering probes

a. X-ray scattering

b. Neutron scattering

1932

1932

1932

V. Findings and Recommendations: LRI in NS

Workshop

1934

A. Recent scientific advances in LRI in NS

1935

B. Challenges and needs in LRI in NS

1935

C. Transformative opportunities from LRI in NS

VI. Conclusions

1936

1936

Acknowledgments

1936

References

1937

\section{INTRODUCTION}

In his famous musing, Plenty of Room at the Bottom, Feynman (1960) noted "... as we go down in size, there are a number of interesting problems that arise. All things do not simply scale down in proportion. There is the problem that materials stick together by the molecular (van der Waals) attractions. It would be like this: After you have made a part and you unscrew the nut from a bolt, it isn't going to fall down because the gravity isn't appreciable; it would even be hard to get it off the bolt. It would be like those old movies of a man with his hands full of molasses, trying to get rid of a glass of water. There will be several problems of this nature that we will have to be ready to design for."

The importance of long range interactions (LRIs) in the synthesis, design, and manipulation of materials at the nanometer scale was thus recognized from the very 
beginning of nanoscience. Yet it is only recently that the intricacies of not only van der Waals forces, referred to by Feynman, but all LRIs have emerged in unexpectedly many research areas. These areas include quantum field theory, quantum and classical density functional theories, various mean-field and strong-coupling statistical mechanical formulations, liquid state integral equations, and computer simulations. These theoretical repercussions lead to novel experimental designs and methods with concomitant novelty and prospects in technology.

The role of LRIs in self-assembling active devices constructed of heterogeneous components is fundamental. They govern the stability of component clusters, essential for the design of nanodevices and nanoactuators. The new technological paradigms that might be developed as a consequence of these fundamental studies promise new ways of thinking that bring old problems closer to solution.

The present review is the outcome of a workshop, Long Range Interactions in Nanoscale Science, convened under the auspices of the United States Department of Energy, Council for the Division of Materials Sciences and Engineering, Basic Energy Sciences. The panel was charged to survey, identify, report, and assess basic research challenges, needs, and opportunities from working with long range interactions. The group examined recent advances in the theory, computation, and measurement of the primary LRIs-electrodynamic, electrostatic, and polar-as well as secondary LRIs, including hydrogen bonding, hydrophobic/hydrophilic/ hydration, steric, structural, and entropic interactions. The aim was to create a comprehensive framework and language of these forces in nanoscience as well as to identify strategies to harness them for the design of new materials and devices.

This endeavor requires spanning a vast range of science from field theory to colloid science, from physical sciences to chemistry and biology, and from theory to experiment and computation. The aim is to couple the fundamentals of LRIs to experimentally accessible systems that can be manipulated on the nanoscale and that can in the foreseeable future enable technological applications. Our review focuses first on the fundamentals of electrodynamic, electrostatic, and polar (acid and/or base) interactions. This focus includes instructive systems that reveal different aspects of LRIs, such as atoms, molecules, nanoscopic, mesoscopic, and macroscopic interfaces, surfaces and defects, as well as chemical equilibria in liquids, suspensions, and colloidal aggregates. We then assess this understanding in order to harness the properties of LRIs in nanoscale systems and to guide the design and chemical construction of electronic, optical, and sensing devices.

The scope of this review is broader than found in a traditional setting. We chose the format to represent the kind of thinking that inspired this conference. It relies on commonality and shared potential of different fields of science so as to weave them into a unifed whole.
TABLE I. Different levels of long range interactions.

\begin{tabular}{lc}
\hline \hline Primary & Secondary \\
\hline & Hydrogen bonding \\
Electrodynamic (van der Waals): & Hydrophobic \\
London, dispersion, Debye, & Hydration \\
Induction, Keesom, orientation & Osmotic \\
Electrostatic (Coulombic) & Disjoining \\
& Structural \\
& Steric \\
& Depletion \\
Polar (acid-base) & Entropy-driven \\
& Enthalpy-driven \\
\hline \hline
\end{tabular}

\section{FUNDAMENTAL INTERACTIONS}

At the most fundamental level, all atomistic interactions are electromagnetic. In spite of this unifying and underlying fundamental principle, various types of atomic and molecular interactions show sufficient specificity either in the underlying theories or in their relative strength within different regimes of interatomic or intermolecular separations. Consequently, they have occupied disjoint scientific communities that study, characterize, classify, and use distinct kinds of interactions in different ways.

The clearest distinction is between electrodynamic van der Waals interactions and electrostatic or Coulomb interactions. Electrodynamic interactions are formulated within the rules of quantum electrodynamics; electrostatic interactions are formulated within the framework of classical electrostatics and statistical mechanics. This fundamental division is subdivided further on an everincreasing ladder of energy and length scales; see Table I.

The limits of this classification are soon encountered, especially when one approaches the nanoscale. The hard edges that used to separate these interactions begin to blur and merge in such a way that their conceptual description and formal models cease to be independent of each other. This leads one to wonder what exactly constitutes a "primary interaction." To some extent this distinction is arbitrary. We selected the van der Waals, the electrostatic, and the acid-base interactions as the primary interactions. All secondary interactions either are subsets of these primary interactions, specific to a class of materials, or are a more macroscopic phenomenon that does not persist down to the atomic interaction level.

The distinction between these long and short ranged basic primary interactions and effective interactions generated by collective phenomena can be properly quantified in terms of the appropriate correlation functions. These correlation functions can be in their turn again 
either short or long ranged. If the underlying primary interactions are long ranged, the correlation functions are often long ranged, but short ranged interactions can nevertheless generate long ranged correlation functions as can be clearly seen close to phase transitions or in the polymer scaling limit. A clear example of this type is the critical Casimir interaction that can be observed close to second-order phase transition (Hertlein et al., 2008). It is important to note here that what constitutes a basic primary long range interaction or an effective long range interaction of course depends crucially on the specific problem under investigation, i.e., on relevant degrees of freedom in the Hamiltonian of the system (electrons, atoms, molecules, etc.).

One of the outcomes of this review was a redefinition of what should be considered "long range" on the nanoscale. On the one hand, gravity can reach out to the cosmos and is certainly a long range interaction, but it is not of the type that would be relevant at the nanoscale. On the other hand, the hydrogen bond that mostly reaches across only a single bond to the next neighbor can qualify as an effective long range interaction since collective phenomena and ensuing effects can extend its range into the nanoscale. Because these effects can propagate well beyond the spatial scale set by nearest neighbors and can affect even the macroscopic properties of materials, one can propose the definition of long range on the nanoscale starting with "extending beyond a single bond."

A viable distinction between the long versus short range interactions would be the algebraically decaying interaction potentials versus exponentially decaying ones. This choice is useful because these classes of interaction potentials lead to qualitatively different properties of resulting correlation functions. However, this definition nevertheless creates a problem since an interaction reveals itself as being long ranged only at large distances where it asymptotically dominates any exponentially decaying short range potential. It thus appears that the distinction between long and short ranged nanoscale interactions is blurred and to some extent idiosyncratic, manifesting itself clearly only at the upper end of the nanoscale or even after entering the mesoscale, with a consequence that on the nanoscale long and short ranged interactions appear to be equally important and difficult to distinguish. Thus here too what constitutes a long range as opposed to short range interaction depends primarily on the specific problem under investigation.

\section{A. Electrodynamic interactions}

Everyday condensed matter is mostly bound by electromagnetic (EM) forces between neutral objects, forces that are animated by electromagnetic fields from the "coordinated dance of fluctuating charges" (Parsegian, 2005). From the atomistic perspective, dilute gases experience these attractive interactions (i.e., the Keesom, Debye, and London dispersion contributions) as a $1 / R^{6}$ function of atomic separation $R$. However, the collective behavior of atoms in condensed matter is better formulated in terms of its macroscopic continuum-dielectric properties. In 1948, by focusing on the quantum fluctuations of the EM field, Casimir computed the force between two parallel ideally metallic plates in vacuum $(\mathrm{Ca}-$ simir, 1948). This approach was later generalized to realistic dielectric materials by Lifshitz, who took into account the fluctuating charge sources in the media (Lifshitz, 1955, 1956; Dzyaloshinskii et al., 1961). It follows from this formulation, later corroborated by experiments (Munday et al., 2009), that the electrodynamic interactions can be attractive as well as repulsive.

The following decades were marked by theoretical advances as well as precise measurements [see, e.g., Sabisky and Anderson (1973) and Derjaguin et al. (1978)]. As the most relevant interaction between neutral bodies at short distance, electrodynamic long range interactions play an important role in, e.g., microelectromechanical systems (Capasso et al., 2007), where they cause metals to attract and to stick at short distances, also known as "stiction" (Serry, 1998).

Given that the theoretical foundation and early experiments were developed in the 1940s and 1950s, why is there now such a burst of activity in electrodynamic"London," "dispersion," "van der Waals," "Casimir," or "Lifshitz"-forces? Was it the advent of high-precision measurements, the ability to manipulate materials, or the technical ability to measure spectra for computation? Unfortunately, it is clear that the different languages and training of those working on different facets of the same problem still impede progress and inhibit constructive collaboration.

Even words pose barriers. The diverse nomenclature of interactions that collectively fall under "electrodynamic" can be separated into two distinct categories: continuum methods using bulk or macroscopic materials properties (Lifshitz, Casimir, etc.) and atomistic [classical force field, density functional theory (DFT), etc.]. These different limits coexist as separate entities and comfortably give meaningful results and insights within their particular regimes. However, the nanotech, biotech, and other popular fields are placing ever-increasing demands upon these formulations so as to be applicable to systems of all sizes and separations, requiring a way to blend them in a straightforward and fundamentally sound manner. This is not easy, but it provides opportunities to develop what is typically seen by outsiders as a "mature" field. The following overview of the foundations of electrodynamic forces includes specific examples of where challenging areas remain.

Though formally not of electrodynamic origin, "critical Casimir interactions" (Krech and Dietrich, 1992; Krech, 1994) might be nevertheless classified with the electrodynamic interactions because they are similar to the original Casimir effect. They arise close to a secondorder phase transition as a consequence of a broken continuous symmetry in the bulk, where the correlation functions acquire an algebraic decay, thus giving rise to bona fide long range effective interactions generated by collective phenomena (Fisher and de Gennes, 1978) on 
top of and in addition to the electrodynamic dispersion force (Dantchev et al., 2007). The formal point of correspondence between the two Casimir effects is that the effective Hamiltonian of a critical fluid corresponds to massless fields and is thus formally equivalent to the electrodynamic Hamiltonian. Though there is formal similarity between standard and critical Casimir interactions, the latter profit from a different type of theoretical approach based on the theory of finite size scaling (Krech and Dietrich, 1991; Maciołek et al., 2007). Critical Casimir interactions present a new pathway to generate long ranged interactions in systems that are nominally governed by short range microscopic interactions.

Critical Casimir interactions are particularly important and have been observed experimentally in the case of thinning of ${ }^{4} \mathrm{He}$ films near the superfluid transition (Garcia and Chan, 1999; Ganshin et al., 2006), near the ${ }^{3} \mathrm{He}-{ }^{4} \mathrm{He}$ tricritical point (Garcia and Chan, 2002; Maciolek and Dietrich, 2006), in binary wetting films (Fukuto et al., 2005), and in colloidal suspensions in the vicinity of chemically patterned surfaces (Soyka et al., 2008). However, in the case of ${ }^{4} \mathrm{He}$ films above and below the superfluid transition, the experimentally observed difference in thickness is larger than can be accounted for by the critical Casimir force. It appears that surface fluctuations of the film surface give rise to an additional force, similar in form but larger in magnitude than the critical Casimir force, which is needed to account quantitatively for the observations (Zandi, Rudnick, and Kardar, 2004).

\section{Lifshitz theory from optical properties}

The van der Waals-London dispersion (vdW-Ld) interactions influence properties ranging from colloidal forces in solution to the fracture of bulk materials. They can significantly affect a given system even when "stronger" forces, such as electrostatic or polar interactions, are acting. One example is the single-wall carbon nanotube separation experiments by Zheng and Semke (2007). Although single-stranded DNA coatings wrap the different chiralities with an equivalent surface charge density, one is able to separate them reliably and repeatably during a salt elution experiment. How could this be if only electrostatics were involved? One theory notes the chirality-dependent optical properties of the single-wall carbon nanotube core. In fact, calculations via the Lifshitz formulation have shown that these differences do exist and can be potentially used in experimental design (Rajter and French, 2008).

At least in principle, proper understanding and a consistent theoretical formulation of the vdW-Ld interaction has been fully achieved within the Lifshitz theory of dispersion interactions (Parsegian, 2005). It provides the link between optical properties or "London dispersion spectra" and the magnitude of these interactions for geometries that are either analytically tractable or easily


b)

FIG. 1. (Color online) A comparison of Lifshitz geometries. (a) Schematic view of the isotropic, plane-plane configuration for the Hamaker coefficient computation between two optically isotropic, semi-infinite half-spaces. (b) Schematic view of the anisotropic, cylinder-cylinder configuration for the Hamaker coefficient computation between two identical single-walled carbon nanotubes $([10,0, s]$ in the case depicted), which is always attractive irrespective of the medium in between, or two different single-walled carbon nanotubes $([10,0, s]$ and $[6,6, m]$ in the case depicted), which can be repulsive in an appropriately chosen intervening medium.

approximated with simpler geometries. For this reason we review the vdW-Ld energy calculations from the bulk-material perspective.

\section{a. Hamaker coefficients}

In the framework of the Lifshitz theory (Parsegian, 2005), the nonretarded dispersion interaction free energy per unit area is

$$
G(l)=-\frac{A_{123}}{12 \pi l^{2}}
$$

where $l$ is the surface to surface separation thickness between two semi-infinite half-spaces (Fig. 1) and $A_{123}$ is the effective Hamaker coefficient, which is defined in this case as 


$$
\begin{aligned}
A_{123} & =-\frac{3}{2} k_{B} T \sum_{n=0}^{\infty} \int_{0}^{\infty} u d u \ln \left[1-\Delta_{32}\left(i \xi_{n}\right) \Delta_{12}\left(i \xi_{n}\right) e^{-u}\right] \\
& \approx \frac{3}{2} k T \sum_{n=0}^{\infty} \Delta_{32}\left(i \xi_{n}\right) \Delta_{12}\left(i \xi_{n}\right)
\end{aligned}
$$

Here 1 and 3 (of subscripts 123) represent the left and right infinite half-spaces separated by medium 2 of thickness $l ; u$ is the magnitude of the wave vector in the plane of the two opposing interfaces. The summation is over a discrete set of Matsubara, or boson, frequencies $\xi_{n}=2 \pi\left(k_{B} T\right) n / \hbar$, where $k_{B}$ is the Boltzmann constant and $2 \pi \hbar$ is the Planck constant. At room temperature, the interval between successive photon energies $\hbar \xi_{n}$ is $\approx 0.16 \mathrm{eV}$. The prime in the summation signifies that the first, $n=0$, term is taken with weight $1 / 2$.

The Hamaker coefficient is largely determined by materials properties. Its magnitude and sign depend on the values of $\Delta$ 's that describe the relative optical spectral mismatches or contrast between neighboring materials at the frequency $\xi_{n}$ involved in the interaction,

$$
\Delta_{i j}\left(i \xi_{n}\right)=\frac{\varepsilon_{i}\left(i \xi_{n}\right)-\varepsilon_{j}\left(i \xi_{n}\right)}{\varepsilon_{i}\left(i \xi_{n}\right)+\varepsilon_{j}\left(i \xi_{n}\right)} .
$$

The dielectric function at imaginary values of the frequency argument $\varepsilon(i \xi)$, the fundamental ingredient of the Lifshitz theory of vdW-Ld interactions, can be obtained via the Kramers-Kronig (KK) transform in the form

$$
\varepsilon(i \xi)=1+\frac{2}{\pi} \int_{0}^{\infty} \frac{\varepsilon^{\prime \prime}(\omega) d \omega}{\omega^{2}+\xi^{2}},
$$

where $\varepsilon^{\prime \prime}(\omega)$ is the imaginary part of the dielectric response function in real frequency space, i.e., $\varepsilon(\omega)$ $=\varepsilon^{\prime}(\omega)+i \varepsilon^{\prime \prime}(\omega) . \varepsilon(i \xi)$ is referred to as the van der WaalsLondon dispersion spectrum. The magnitude of $\varepsilon(i \xi)$ describes how well the material responds and is polarized by fluctuations up to a given frequency. While the integration in Eq. (4) requires spectra to infinite frequencies, in practice this is unnecessary as long as all interband transition energies are either known or properly approximated. For simple systems, it may be acceptable to use this simple formulation for planar geometries to estimate the magnitude and sign of the Hamaker coefficient. However, there are situations where the geometry can also influence the Hamaker coefficient by altering the form of $\Delta_{i j}$ (Rajter et al., 2007).

\section{b. Full spectral optical properties}

Traditionally, optical spectra were obtained either experimentally or approximated using damped-oscillator models. Recently it has been possible to use $a b$ initio calculations. The particular method used is relevant only if caveats are considered, e.g., particular frequency or energy ranges where data are not trustworthy. Sometimes it is possible to adequately approximate gaps in spectral properties, such as using analytical wings and oscillator extensions (French, 2000). Once sufficiently obtained and/or approximated, the various experimental or $a b$ initio optical properties are interchangeable and can be directly transformed into the required vdW-Ld spectra using the appropriate Kramers-Kronig (KK) transforms. For example, valence electron energy loss spectrum measurement might give the frequencydependent results in $J_{c v}(\mathrm{eV})$ (interband transition strength form in $\mathrm{eV}$ frequency units) while the $a b$ initio codes give the imaginary part of the dielectric spectrum over real frequencies $\varepsilon^{\prime \prime}(\omega)$. One can convert between the two via

$$
J_{c v}(\omega)=\frac{m_{0}^{2} \omega^{2}}{e^{2} \hbar^{2} 8 \pi^{2}}\left[\varepsilon^{\prime \prime}(\omega)+i \varepsilon^{\prime}(\omega)\right] .
$$

From this, one can use $\varepsilon^{\prime \prime}(\omega)$ in the KK transform [Eq. (4)] to determine the vdW-Ld spectra.

\section{c. Model systems: $P S$-water-PS and $\mathrm{SiO}_{2}$-water-SiO ${ }_{2}$}

The many layers of abstraction within the Lifshitz formulation can hide the linkage between optical properties and underlying material properties. Knowing how optical properties depend on the underlying material composition and crystal structure can benefit materials design and engineering.

For example, the interband optical properties of polystyrene in the vacuum ultraviolet (VUV) can be investigated using combined spectroscopic ellipsometry and VUV spectroscopy (French et al., 2007). Over the range $1.5-32 \mathrm{eV}$, the optical properties exhibit electronic transitions that can be assigned to three groupings, corresponding to a hierarchy of interband transitions of aromatic $\left(\pi \rightarrow \pi^{*}\right)$, nonbonding $\left(n \rightarrow \pi^{*}, n \rightarrow \sigma^{*}\right)$, and saturated $\left(\sigma \rightarrow \sigma^{*}\right)$ orbitals. In polystyrene there are strong features in the interband transitions arising from the side-chain $\pi$ bonding of the aromatic ring consisting of a shoulder at $5.8 \mathrm{eV}$ and a peak at $6.3 \mathrm{eV}$, and from the $\sigma$ bonding of the $\mathrm{C}-\mathrm{C}$ backbone at 12 and $17.1 \mathrm{eV}$. These transitions have characteristic critical point line shapes associated with one-dimensionally delocalized electron states in the polymer backbone. A small shoulder at $9.9 \mathrm{eV}$ is associated with excitations possibly from residual monomer or impurities.

Having obtained polystyrene's optical spectra from the above experiments, it is then trivial to calculate Hamaker coefficients and vdW-Ld interaction energies in the plane-parallel geometry (Fig. 1) at all separation distances. One can also vary the intervening medium (by changing its full spectral optical properties) in order to get the Hamaker coefficients and the vdW-Ld component of the surface free energy for polystyrene immersed in these other materials; see Table II.

Similar and related calculations and analysis can also be done for two semi-infinite slabs of $\mathrm{SiO}_{2}$ interacting across various media (Tan et al., 2005). The interband optical properties of crystalline quartz and amorphous $\mathrm{SiO}_{2}$ in the VUV have been investigated using combined spectroscopic ellipsometry and VUV spectroscopy. Over the range of $1.5-42 \mathrm{eV}$, the optical properties exhibit similar exciton and interband transitions, 
TABLE II. Full spectral Hamaker coefficients for vdW-Ld interactions of different physical configurations with polystyrene, amorphous $\mathrm{SiO}_{2}$, or water, using interband transition strength spectra.

\begin{tabular}{|c|c|c|c|c|c|}
\hline Physical geometry & $\begin{array}{l}\text { Hamaker } \\
\text { coeff. }[\mathrm{zJ}]^{\mathrm{a}}\end{array}$ & Physical geometry & $\begin{array}{l}\text { Hamaker } \\
\text { coeff. }[\mathrm{zJ}]\end{array}$ & Physical geometry & $\begin{array}{l}\text { Hamaker } \\
\text { coeff. }[\mathrm{zJ}]\end{array}$ \\
\hline$[\mathrm{PS} \mid$ vacuum $\mid \mathrm{PS}]$ & 70.9 & {$\left[\mathrm{SiO}_{2} \mid\right.$ vacuum $\left.\mid \mathrm{SiO}_{2}\right]$} & 71.6 & [water|vacuum|water] & 34.2 \\
\hline [PS|water|PS] & 7.71 & {$\left[\mathrm{SiO}_{2} \mid\right.$ water $\left.\mid \mathrm{SiO}_{2}\right]$} & 8.06 & & \\
\hline$\left[\mathrm{PS}\left|\alpha-\mathrm{SiO}_{2}\right| \mathrm{PS}\right]$ & 1.53 & {$\left[\mathrm{SiO}_{2}\left|\mathrm{SiO}_{2}\right| \mathrm{SiO}_{2}\right]$} & 0 & {$\left[\right.$ water $\left|\mathrm{SiO}_{2}\right|$ water $]$} & 8.04 \\
\hline \multicolumn{6}{|c|}{ Literature results [PS|water|PS] reported by Bos et al. (1999) } \\
\hline $\begin{array}{l}\text { [PS|water|PS }] \\
\quad \text { from contact angles }\end{array}$ & 5 & $\begin{array}{l}\text { [PS|water|PS }] \text { from } \\
\text { macroscopic measurements }\end{array}$ & $3.5-5.3$ & & \\
\hline $\begin{array}{l}\text { [PS|vacuum|PS] } \\
\quad \text { from Dagastine et al. }(2004)\end{array}$ & 8.90 & $\begin{array}{c}\text { [PS|vacuum|PS] from } \\
\text { Dagastine et al. (2004) using } \\
\text { Parsegian and Weiss (1972) PS }\end{array}$ & 9.63 & & \\
\hline
\end{tabular}

${ }^{\mathrm{a}} 1 \mathrm{zJ}=10^{-21} \mathrm{~J}$.

with crystalline $\mathrm{SiO}_{2}$ exhibiting larger transition strengths and indices of refraction. Crystalline $\mathrm{SiO}_{2}$ has more sharp features in the interband transition strength spectrum than amorphous $\mathrm{SiO}_{2}$, the energy of the absorption edge for crystalline $\mathrm{SiO}_{2}$ is about $1 \mathrm{eV}$ higher than that for amorphous $\mathrm{SiO}_{2}$, and the direct band-gap energies for $X$ - and $Z$-cut quartz are 8.30 and $8.29 \mathrm{eV}$ within the absorption coefficient range $2-20 \mathrm{~cm}^{-1}$.

By calculating and analyzing the Hamaker coefficients, we can determine the degree to which changes in crystal structure or composition influence the vdW-Ld interaction. Comparing $c-\mathrm{SiO}_{2}$ or $a-\mathrm{SiO}_{2}$, there is an appreciable increase in the overall vdW-Ld strength for the $c-\mathrm{SiO}_{2}$, which is a result of its increased physical density, index of refraction, transition strengths, and oscillator strengths compared with $a-\mathrm{SiO}_{2}$ (Table I).

\section{d. Advanced effects and phenomenology}

There are many other effects that occur as we make the system more complex. The first is that the Lifshitz formulation allows for attractive as well as repulsive Hamaker coefficients, based on the magnitude and spatial arrangement of the optical contrast at each interface in the system. One example of this common phenomenon is liquid helium films where the substrate material prefers as much contact as possible with helium rather than with air (Sabisky and Anderson, 1973). There is "complete wetting" that occurs because the system prefers to make the medium as thick as possible.

Wetting phenomena are relevant in the context of LRIs not only in the framework of liquid helium, but from a general point of view. On the nanoscale they are very sensitive to the range of the underlying interactions in two ways. First, there is a qualitative difference between short and long range forces in that for the former capillary wavelike fluctuations are relevant, whereas for the latter these fluctuations are not. Second, the dependence of wetting film thickness on thermodynamic variables, e.g., pressure, directly reveals the algebraic decay behavior of the underlying forces (Dietrich, 1988). In a similar vein, the LRIs also give rise to algebraically de- caying (van der Waals) tails in the density profiles of thin fluid films (Dietrich and Napiórkowski, 1991).

In the context of wetting one should also note that LRIs translate nanosculptured substrate morphologies into distinct wetting properties (Gang et al., 2005; Tasinkevych and Dietrich, 2006, 2007).

The next effect is that of retardation and/or sign reversal. Because of the finite speed of light, the highenergy frequency contributions to the summation in Eq. (2) dampen quickly as the separation distance increases. Thus the Hamaker coefficient itself has a nonlinear dependence on separation. For example, consider a system where the higher-energy part of the spectrum gives rise to primarily repulsive terms and the low-energy part contributed attractive terms. At large separations, where retardation eliminates all but the low-energy terms, the overall interaction is positive. However, as the particles attract and get closer, the Hamaker coefficient would continue to add repulsive terms to the overall summation until it hits zero and actually changes sign. This combination of attractive and repulsive contributions can lead to interesting effects, such as an equilibrium 1-2 nm vdW-Ld separation energy well and the existence of premelting in surface layers of ice (see Sec. II.C.1).

Multilayers (such as coatings) experience a set of competing interactions because the effective optical properties of a layered object can change drastically as a function of separation distance (Podgornik et al., 2006). Optical anisotropy will create a configurational or directional component such as to cause alignment forces and torques (Parsegian and Weiss, 1972). Many such effects, important on the nanoscale, are only beginning to be recognized, probably because the necessary optical properties and extensions to the Lifshitz formulations are still under development.

We also note that although this review is devoted to static structural LRI dependent properties, there are well-known specific influences of LRIs on the dynamics of nanoscale objects. As an example, note that under the action of the long ranged tails of long range dispersion 


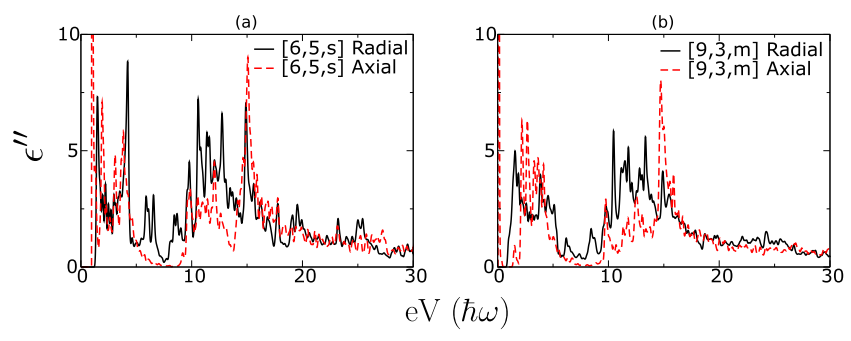

FIG. 2. (Color online) Ab initio dielectric function $\left(\varepsilon^{\prime \prime}\right)$ spectra for the (a) semiconducting $[6,5, s]$ and (b) metallic $[9,3, m]$ SWCNTs in the radial and axial directions from 0 to $30 \mathrm{eV}$. Each spectra depends on direction and chirality. From Rajter et al., 2007.

forces nanodroplets move in lateral directions which are opposite to the ones expected for micron-sized drops (Moosavi et al., 2006, 2008a, 2008b).

\section{Ab initio optical properties of complex materials}

The previous sections outlined the important effects that result from even small changes in the full spectral optical properties. Given the ubiquity of vdW-Ld interactions in condensed matter, one might naively assume that there is a large catalog of these spectra available. This information could then be data-mined and used in all sorts of ways, particularly in experimental design.

Unfortunately, obtaining full optical spectra is a more difficult endeavor than most realize. While vacuum ultraviolet spectroscopy and valence electron energy loss spectroscopy (V-EELS) are well established and have proven critical to reliable force computation (French, 2000; French et al., 2007), stringent sample preparation specifications (among other factors) make a generalized cataloging of hundreds of materials cost and time prohibitive. Liquids are particularly difficult to characterize under the required vacuum conditions with consequent problems in containment. To experimentally characterize spatially varying, deep UV optical properties of a complex material in a liquid medium would be difficult or impossible.

More recently, $a b$ initio codes have proven to be a viable alternative to analyze those materials whose spectra cannot be cleanly obtained experimentally (e.g., biological molecules immersed in a liquid solution). In order to capture all possible electron transitions between the valence and conduction bands of the total electronic structure, these orthogonalized linear combination of atomic orbitals-DFT codes use very large basis sets. Test calculations on several ceramic crystals showed that the calculated Hamaker coefficients using theoretical spectra do not differ much from those obtained using experimental spectra (Ahuja et al., 2004). Recently this approach has been applied to obtain Hamaker constants for both metallic and semiconducting single-wall carbon nanotubes (SWCNT) and multiwall carbon nanotubes (MWCNT) of different chiralities with considerable success (Rajter, French, et al., 2008; Rajter et al., 2008).

In Figs. 2 and 3 we show $\varepsilon^{\prime \prime}(\omega)$ and the corresponding

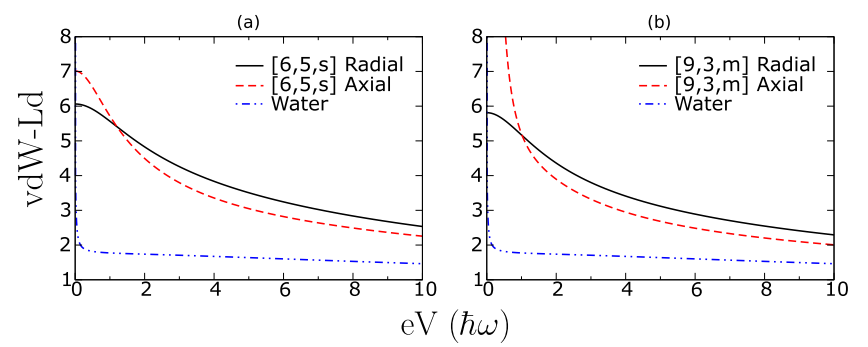

FIG. 3. (Color online) The van der Waals-London dispersion spectra for the (a) semiconducting $[6,5, s]$ and (b) metallic $[9,3, m]$ SWCNTs in the radial and axial directions from 0 to $30 \mathrm{eV}$. The differences are large enough to create chirality and orientation-dependent vdW-Ld interactions. From Rajter et al., 2007.

vdW-Ld spectra properties of $[6,5, s]$ and $[9,3, m]$ SWCNTs. It is important to capture all of these interband transitions, out to at least $30 \mathrm{eV}$, because all areas are adding to the overall summations and can shift the magnitude of the resulting Hamaker coefficients. vdW-Ld interactions require accurate information for electronic transitions well beyond the band gap, while device performance studies have typically been focused near the band gap energy. But beyond a 30-40 eV cutoff, the transitions become less important because they are considerably dampened by the KK transform and therefore need to be much larger in order to be significant.

While the ascertaining and usage of $a b$ initio optical properties for vdW-Ld interactions is still a new and small field, its speed, low cost, and broad utility makes it an appealing solution to the long-standing problem of a dearth of optical property catalogs. It even provides exciting new possibilities, such as spatially resolving the optical properties for, e.g., biological materials, which will be described in Sec. III.A.1.

\section{Electrodynamic interactions for arbitrary shapes}

The impact of shape or geometry is a more complex but equally important component in determining the multibodied behavior of electrodynamic LRIs. Recall that the sources of these LRIs arise from the quantum fluctuations of the electromagnetic (EM) field as they are modified by the presence, positions, and shapes of metallic (Casimir) or dielectric (Lifshitz) objects (Casimir, 1948; Lifshitz, 1956). The advent of high-precision measurements (Lamoreaux, 1997; Mohideen and Roy, 1998; Roy et al., 1999), and the possibility that they can be applied to nanoscale electromechanical devices (Serry et al., 1998; Chan et al., 2001; Decca et al., 2003), has stimulated interest in developing a practical way to calculate the dependence of electrodynamic energies on the shapes of the objects.

The simplest and most commonly used methods for dealing with complex shapes rely on pairwise summations. In the proximity force approximation (PFA), also referred to as the Derjaguin approximation, the energy is obtained as an integral over infinitesimal parallel sur- 

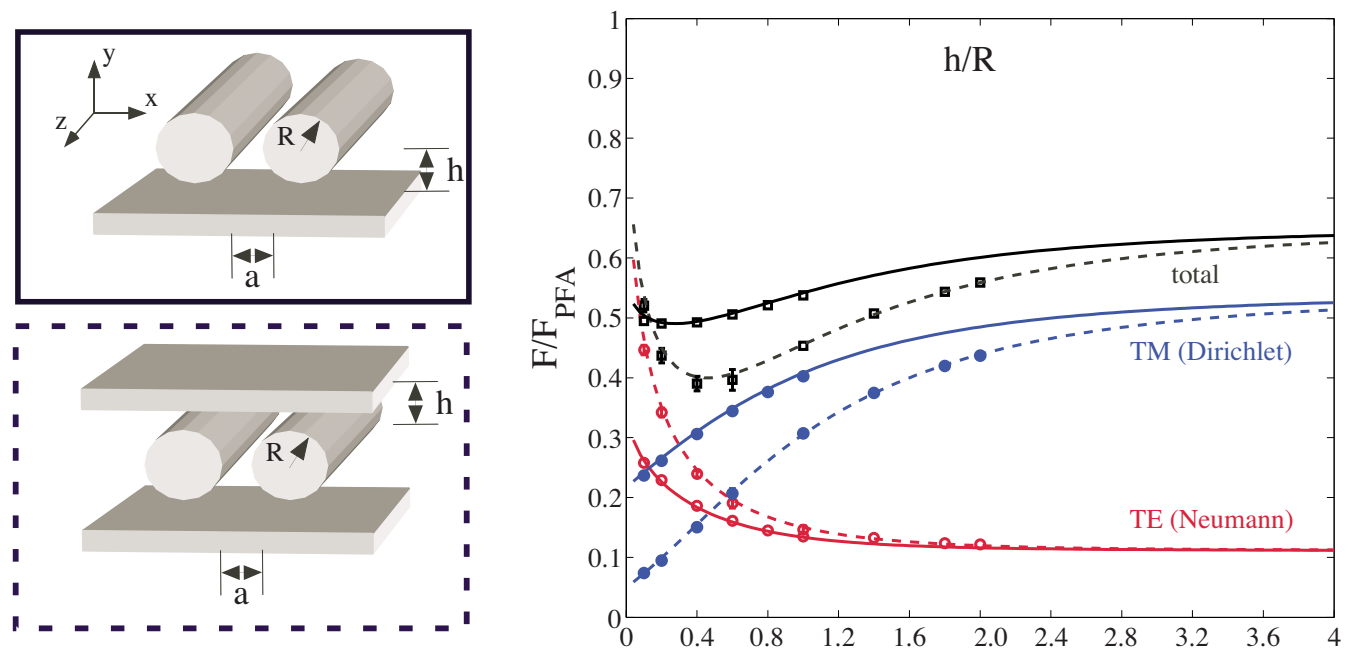

FIG. 4. (Color online) Casimir force per unit length between two cylinders (black) vs the ratio of sidewall separation to cylinder radius $h / R$, at fixed $a / R=2$, normalized by the total "proximity force approximation" force per unit length between two isolated cylinders. From Rahi et al., 2008.

face elements at their local separation measured perpendicular to a surface (Rempel et al., 2001; Parsegian, 2005) and works well when two objects are very close together as, for example, in the case of SWCNTs at small separations (Rajter et al., 2007). A second method described by Sedmik et al. (2007) is based on addition of CasimirPolder "atomic" interactions (CPI). Unfortunately, both methods are heuristic and not easily amenable to systematic improvements.

Balian and Duplantier (1978) proposed a method, based on a multiple scattering expansion, for calculating Casimir energies for arbitrary shape. However, this method has not proved workable in practice. An approach based on path integral methods has also been used to compute corrections to the parallel-plate result by perturbation for small deformations (Emig et al., 2003). The limitations of perturbation theory weaken the usefulness of this approach, but in some cases it can be overcome by specialized numerical methods (Büscher and Emig, 2005). There is also a numerical implementation of the path integral method (Gies and Klingmuller, 2006), which has so far been applied only to scalar fields. Recent numerical approaches have been based either on an explicit discretization of the EM fields in space and computation of the mean stress tensor (Rodriguez, Ibanescu, Iannuzzi, Capasso, et al., 2007) or on the boundary element method by rewriting the van der Waals interaction energy exclusively in terms of surface integrals of surface operators (Veble and Podgornik, 2007a). In principle, both schemes can deal with arbitrary geometries and a spatially varying dielectric constant.

\section{a. Cylinders and plates}

The geometry of the cylinder, as intermediate between sphere and plate, is ideally suited to testing the limitations of PFA and CPI approximations. It is also relevant to important experimental systems such as carbon nanotubes [see Fig. 1(b)] and stiff polymers such as
DNA. The translational symmetry along the cylinder axis considerably simplifies the problem, as the electromagnetic (EM) field (with metallic objects) can be decomposed into transverse magnetic (TM) and transverse electric (TE) components, with Dirichlet and Neumann boundary conditions, respectively. Emig et al. (2006) exploited this to find the exact Casimir force between a plate and a cylinder, and there have been further elaborations (Brown-Hayes et al., 2005; Bordag, 2006). The force has an unexpectedly weak decay

$$
F(H) \propto \frac{L}{H^{3} \ln (H / R)}
$$

at large plate-cylinder separations $H$ ( $L$ and $R$ are the cylinder length and radius), due to transverse magnetic modes. Path integral quantization with a partial wave expansion additionally provides the density of states, and corrections at finite temperatures. An example of the failure of pairwise additivity was obtained by Rodriguez, Jesse, et al. (2007), in which the Casimir force between two squares exhibits a nonmonotonic dependence on the distance from enclosing sidewalls. Figure 4, left panel, shows the same effect for two cylinders, with one or two nearby sidewalls (all metals). The right panel of Fig. 4 shows results for the force between two cylinders, normalized by the PFA result. The solid and dashed lines correspond to one and two sidewalls, respectively; in each case the contributions of TM and TE modes to the total force are also depicted. For the plotted separation of $a / R=2$, PFA overestimates the force by roughly a factor of 2 . More significantly, in pairwise approximations (PFA or CPI) the sidewall(s) have no effect on the force between the cylinders. The nonmonotonic variation with the separation $H$ to the plates is thus a direct illustration of the importance of three-body effects. 


\section{b. Spheres and compact shapes}

A potentially quite powerful approach for computing electrodynamic forces for materials of arbitrary shape and composition was developed recently (Emig et al., 2007). There are dual equivalent perspectives on the Casimir interaction: in terms of the fluctuating EM field or the fluctuating sources (charges and currents) in the material bodies (Schwinger, 2004); Emig et al. employ the latter. The fluctuating sources on the different objects (labeled by greek letters) are indicated by $\left\{Q_{\alpha}\right\}$. Each $Q_{\alpha}$ carries multiple indices that designate the source (charge or current), partial wave $(l, m)$ in a multipole representation, and frequency (after Fourier transformation in time), which will be suppressed. In path integral quantization, each configuration is weighted using an action $S\left[\left\{Q_{\alpha}\right\}\right]$, which is quadratic and comprised of several parts. The off-diagonal elements of the action,

$$
S\left[\left\{Q_{\alpha}\right\}\right]=Q_{\alpha} V_{\alpha \beta} Q_{\beta},
$$

represent the interaction between charges. As is familiar from electrostatics, we expect that the lowest multipoles dominate the interaction at large separations. The matrix elements $V_{\alpha \beta}$ are thus a function of the separation $D_{\alpha \beta}$ and the implicit multipoles. The "diagonal" components,

$$
S\left[\left\{Q_{\alpha}\right\}\right]=\frac{1}{2} Q_{\alpha} T_{\alpha}^{-1} Q_{\alpha},
$$

are more interesting and represent the self-energy (action) of the source. The crucial observation is that the matrices $T_{\alpha}$, which encode all relevant shape and material properties of the objects, are directly related to scattering from the object (Newton, 1966). This connection was also noted by Kenneth and Klich (2006), and provides a link to the mature and well-developed field of scattering of EM waves from different objects.

The $T$ matrix can be obtained for dielectric objects of arbitrary shape by integrating the standard vector solutions of the Helmholtz equation in dielectric media over the object's surface (Waterman, 1971), and both analytical and numerical results are available for many shapes (Mishchenko et al., 2004). For the specific case of two dielectric spheres, for which explicit formulas for the $T$ matrix are available, the Casimir force can be obtained at all separations. Focusing on low-order multipoles gives an expansion in powers of the ratio of sphere radius to separation $(R / D)$. Due to alternating signs, the convergence of this series is problematic, but a convergent approach can be obtained by including all terms coming from a given order in the multipole order 1 (irrespective the power in $R / D$ ), and extrapolating to 1 $\rightarrow \infty$. This procedure yields a curve for the force that interpolates all the way from the Casimir-Polder limit to the PFA result at short separations.

This concludes our basic overview of the fundamentals of continuum model, electrodynamic LRIs. We now turn our attention to the atomistic methods, specifically the wide variety available using ab initio quantum codes.

\section{Noncovalent interactions from electronic structure calculations}

Approximate numerical solutions to the electronic Schrödinger equation have become a standard tool for $a b$ initio prediction of materials properties in the fields of computational physics, chemistry, and biology. Quantum Monte Carlo and post Hartree-Fock methods, such as coupled cluster or configuration interaction, are able to reach an accuracy which is more than sufficient for comparison to spectroscopic experiments. They suffer, however, from a prohibitive computational cost with an increasing number of electrons. Alternatively, the solution of the Schrödinger equation within the Kohn-Sham density functional theory (KS-DFT) framework (Hohenberg and Kohn, 1964; Kohn and Sham, 1965; Parr and Yang, 1989) frequently proves to represent not only a reasonable trade-off between accuracy and computational cost (Koch and Holthausen, 2001), but also a conceptually more appealing view on the electronic manybody problem in terms of the single-particle electron density, $n(r)$. Furthermore, the modest computational cost of DFT led to the development of ab initio molecular dynamics methods such as Born-Oppenheimer or Car-Parrinello molecular dynamics (Iftimie et al., 2005). In principle, KS-DFT is an exact theory that yields the exact electronic ground state and interatomic potential. In practice, however, the exchange-correlation energy $E_{\mathrm{xc}}[n(r)]$, the unknown term in the KS-Hamiltonian, must be approximated, thereby rendering the accuracy less than perfect.

DFT has been widely successful in describing the properties in dense materials and isolated molecules, where local and semilocal approximations and their generalizations typically give satisfactory results (Staroverov et al., 2003, 2004); hybrid functionals (Sousa et al., 2007) play a special role in the molecular case. However, sparse systems, soft matter, molecular van der Waals complexes, biomolecules, and the like cannot be adequately described by the previously standard DFT approximations. These "weak" LRIs are among the phenomena for which the first generations of approximations to the exchange-correlation interaction $v_{\mathrm{xc}}$, the local density and generalized gradient approximations, LDA and GGA, yielded qualitatively erroneous predictions. Kristyán and Pulay (1994) and PérezJordá et al. (1994) showed this more than a decade ago for rare gas, as did Meijer and Sprik (1996) for the benzene dimer and benzene crystal. For a recent assessment of the performance of 44 approximations to $v_{\mathrm{xc}}$ for describing nonbonded dimers see Zhao and Truhlar (2005a). These difficulties have now become widely recognized, and a number of different techniques are being developed and exploited to deal with the situation where the LRIs are important. We start by reviewing these new techniques. We then discuss additional approaches of incorporating vdW-Ld alongside DFT. These approaches represent only a beginning to the solution of a critical problem. 
It is also necessary to point out that there exists not only an electronic density functional theory, but a sophisticated and properly formulated classical density functional theory describing the structural properties of inhomogeneous classical liquids on the molecular and nanoscale (see Evans, 1979, 1992).

\section{a. Recently evolving density functional theory methods}

We characterize emerging DFT methods as (i) manybody, using Kohn-Sham orbitals; (ii) empirical and nonempirical explicit density functional; and (iii) perturbative, which leave the electronic structure uncorrected and aim solely at predicting the correct interatomic potentials, usually requiring the input of $C_{6}$ coefficients, the polarizabilities, or damping functions which are consistent with the employed exchange-correlation interaction $v_{\mathrm{xc}}$. This characterization scheme is to some extent arbitrary. Another scheme might be according to whether or not it would be necessary to identify distinct fragments of matter in order to define and apply the method; this would split the methods in each of the categories above.

i. Many-body methods using Kohn-Sham orbitals. We begin by discussing an important type of method whose use has recently expanded and is based on the random phase approximation (RPA), often enhanced in various ways, such as using corrections in the style of time-dependent density functional theory to the RPA density-density correlation function. The RPA approximates long range correlations including van der Waals, but when applied to uniform systems it is less accurate than the modern form of LDA based on fitting of exact limits and quantum Monte Carlo simulations, so it was abandoned decades ago as a DFT technique. A suggestion by Kurth et al. (1999) to simply apply the full RPA and to correct the above error with an extra local or semilocal correction seems to have resurrected its use. RPA methods have recently been applied not only to model systems (Dobson and Wang, 1999; Pitarke and Perdew, 2003; Jung et al., 2004), but also to molecules (Aryasetiawan et al., 2002; Fuchs and Gonze, 2002; Furche and Van Voorhis, 2005) and solids (Miyake et al., 2002); many of these are not obviously vdW systems, but of recent interest is the application by Marini et al. (2006) to a vdW-bonded layered solid (BN). By separating the layers, the system can be brought into the region where vdW-Ld is predominant, where both the strength and weakness of the method is shown. Its strength is that there is no empirical input, distinct fragments do not need to be defined, and it can be applied to both finite and extended systems. Its weakness is shown by the error bars: it is computationally intensive; one must calculate excited KohnSham states accurately, which implies either a fine grid or a large basis set, depending on the method used.

Symmetry-adapted perturbation theory (SAPT) is a quantum chemical method which treats the interaction between monomers via perturbation theory. Years ago a method was introduced that mimics the SAPT procedure using KS orbitals (Williams and Chabalowski,
2001), which we term SAPT-DFT. The initial version was rather inaccurate, but various improvements, some of which are semiempirical, have yielded a method capable of giving good results (Heßelmann and Jansen, 2003; Misquitta et al., 2003). However, there are also weaknesses. First, the method has not been developed for application to extended systems. For finite systems, although the scaling with system size is superior to the state-of-the-art coupled-cluster wave function methods, it is nevertheless significantly worse than for either standard DFT methods or the recent DFT methods that include vdW-Ld, as discussed below. Its application necessitates the identification of individual fragments, so it cannot be seamless as fragments merge together to become single entities.

ii. Explicit density functional methods. The exchange and correlation energies are given as explicit functionals; this means specifically that once the occupied Kohn-Sham orbitals and hence the density are obtained, the exchange and correlation energy components are simply evaluated, without the need to calculate unoccupied KS orbitals. This requirement guarantees that the scaling of the computational requirements with system size will not destroy the cubic scaling enjoyed by ordinary DFT.

The modern version of the functional nonempirical vdW-DF was introduced several years ago (Dion et al., 2004), with the fully self-consistent version (Thonhauser et al., 2007) coming more recently. It is completely consistent with the result stemming from a properly reformulated Lifshitz theory (Veble and Podgornik, 2007b). The new version supplants the obsolete functional for planar systems (Rydberg et al., 2000). Like the RPA methods, the correlation functional of vdW-DF is nonempirical. Unlike them, however, it does not automatically provide its own exchange functional. For this the revised Perdew-Burke-Ernzerhof (revPBE) functional (Zhang and Yang, 1998) version of the generalized gradient approximation (GGA) was used, because it appeared to give the best agreement with Hartree-Fock calculations when the correlation functional was omitted. The vdW-DF method has shown promise for a variety of system types where vdW-Ld interactions are important (Kleis et al., 2007; Thonhauser et al., 2007; Chakarova-Käck et al., 2008; Cooper et al., 2008). The method appears to be most accurate for larger systems, where the multiplicity of probable particle-hole excitations more closely matches the assumptions under which it was derived. Even for small systems like rare-gas dimers, it qualitatively captures the vdW-Ld interaction, which is missed by standard density functionals. It can handle extended systems with large unit cells, as well as large finite systems, as its scaling with system size is the same as ordinary DFT. It is fully self-consistent, which means that it can produce the Hellmann-Feynman internuclear forces that are crucial for relaxation and molecular dynamics. It does not require the identification of fragments, and if they physically exist, the theory is seamless as they merge. Prototypical results, using this approach for large systems, include physisorption of benzene and naphthalene on graphite, the structure and 
binding of a polyethylene crystal, and a DNA base-pair dimer, whose sequence-dependent twist matched trends from high-resolution data (Olson et al., 1998).

One can aim to improve the electron-electron interaction such that the corrected electron density yields correct atomic forces. This is tantamount to improving $v_{\mathrm{xc}}$ empirically, as it has already been done for functionals which yield reasonable atomic and intramolecular energies. Usually, a small set of parameters is fitted to many reliable reference results, in this case nonbonded complexes. The recently introduced X3LYP functional (Xu and Goddard, 2004) was quickly shown to fail completely for stacked nucleic acid bases and amino acid pairs (Cerny and Hobza, 2005), although hydrogen bonding was found to be well described. It thereby follows the typical pattern of conventional density functionals that fail to properly describe the dispersion interaction. Zhao and Truhlar (2005a, 2005b) presented various empirical functionals for weak interactions.

Alternatively, inspired by the idea that molecular properties can be influenced through the parametrization of effective core potentials (Hellmann, 1935), an electron-nucleus correction, "London dispersioncorrected atom-centered potentials" (DCACP), has been introduced (von Lilienfeld et al., 2004). In that approach a set of parameters, $\left\{\sigma_{i}\right\}$, in an atom-centered potential is calibrated for every atom I in the periodic table and added to a GGA exchange-correlation potential,

$$
v_{\mathrm{xc}}=v_{\mathrm{xc}}^{\mathrm{GGA}}+\sum_{I} v_{I}^{\mathrm{DCACP}}\left(\left\{\sigma_{i I}\right\}\right)
$$

Conventionally, the DCACP has the functional form of the analytical pseudopotentials proposed by Goedecker et al. (1996). Current versions of this correction use two $\sigma$ parameters per atom which are calibrated to experimental or highly accurate theoretical results for prototypical van der Waals complexes. Thereafter, the same atomic potential is employed for the different chemical environments. The latest generation of calibrated potentials can be found by Lin, Coutinho, et al. (2007). This scheme has already successfully been applied to a range of systems and situations, such as small rare-gas clusters, the hydrogen bromide dimer, and conformational changes in cyclooctane (von Lilienfeld, Tavernelli, et al., 2005) to the adsorption of Ar on graphite (Tkatchenko and von Lilienfeld, 2006), to the coarse graining of intermolecular potentials of discotic aromatic materials (von Lilienfeld and Andrienko, 2006), to the dimers of small organic molecules, and to cohesive energies and the lattice constant of the benzene crystal (Tapavicza et al., 2007), to the intermolecular binding of DNA base-pairs and base-pair or intercalator drug candidate (Lin, von Lilienfeld, et al., 2007), and liquid water. However, for all these empirical approaches to the exact form of the exchange interaction $v_{\mathrm{xc}}$, the fundamental problem remains that a priori not only the parameters in $v_{\mathrm{xc}}$ are unknown but even its functional form.

\section{b. $C_{6}$ coefficients of polarizabilities, and damping functions}

We use the term DFT-damped $C_{6}$ to describe any of the methods that treat short range interactions by DFT, but treat vdW-Ld via a damped interaction directly between atomic nuclei. This is based on London's original perturbational work yielding the $C_{6} / R^{6}$ asymptotic dissociative scaling between two atoms at distance $R$ (Heitler and London, 1927). The total energy is then extended by

$$
\sum_{I<J} C_{6 J} C_{6 I} / R_{I J}^{6}
$$

and must be damped to avoid any spurious effects on the repulsive potential which originates in the nuclear and electronic Coulomb, and the Pauli repulsion. The vdW-Ld interaction is thus not treated by DFT at all, but rather by a force-field method with a dissociative Lennard-Jones potential. There exists a multiplicity of such methods (Elstner et al., 2001; Wu et al., 2001; Wu and Yang, 2002) that generally require a substantial amount of empirical input. Recently, Grimme and coworkers systematically tabulated $C_{6}$ coefficients for usage in various GGA functionals. They employed them for studies of supramolecular host guest systems (Parac et al., 2005), for organic reactions involving anthracene, for supramolecular aggregates of bio-organic compounds (Grimme et al., 2007), and even fullerenes and graphene sheets. Ortmann et al. (2005) used the same $C_{6} / r^{6}$ approach to study the adsorption of a DNA base on graphite, as well as solid state properties (Ortmann et al., 2006). While they do find significant improvement for isolated dimers or surface adsorption, condensed phase properties such as the lattice constants and the bulk moduli of $\mathrm{Ne}$ and Ar crystals show no improvement at all. This is discouraging because it indicates an intrinsic limit to the applicability of the $C_{6}$ correction when dealing with all condensed phase systems. One of the reasons for the failure to improve upon crystal properties is that many-body contributions to the vdW-Ld forces themselves in the condensed phase are not accounted for correctly here (Tkatchenko and von Lilienfeld, 2008). Yet, because of their low computational requirements, methods of this type are used by many groups.

The $C_{6}$ coefficients can be determined from atomic static polarizabilities (Parsegian, 2005), on-the-fly for atoms in molecules using scaled atomic volumes and polarizabilities (Tkatchenko and Scheffler, 2009), from electronic excitations (Marques et al., 2007), or from the exchange-correlation hole as proposed by Becke and Johnson (2005). The latter method has been applied as the dispersion energy part of a density functional (Becke and Johnson, 2005; Johnson and Becke, 2006). It has some similarities to DFT-damped $C_{6}$, but with less semiempirical input, and more importantly with a carefully reasoned, although heuristic argument for its validity. This method requires the monomer static polarizabilities as input, much like the early asymptotic functionals that either required these polarizabilities (Hult et al., 1998) or 
a cutoff that determined them (Andersson et al., 1996), the $C_{6}$ coefficient, and eventually higher-order $C$ 's. However, instead of the multiparameter damping functions used in DFT-damped $C_{6}$ type theories, it was found that a single universal parameter could be used.

\section{Challenges and opportunities}

The encouraging progress of the past several years only makes it clearer where more work must be done. We are still learning the importance of shape and topology. We are learning the consequences of material properties, especially as they are modified by temperature, pressure, defects, and impurities. On the longest scales, materials can be approximated as continua with emphasis on the consequences of shape and orientation. On the shortest scales, we must recognize atoms and molecules. Here the need is for accurate $a b$ initio approaches and density functional methods. On intermediate scales, we need effective potentials that capture the crossover between the atomic and the continuum and that can enable efficient and accurate numerical simulations.

Although the foundations of the modern day continuum models were laid over 50 years ago, many still prefer to employ and to speak in terms of the even earlier (1930s) picture that made little conceptual distinction between gasses and condensed materials. Much of this resistance to embracing the Lifshitz or Casimir formulations comes from the perception that they are too unwieldy or difficult to use. Paradoxically modern formulations are in many ways simpler than the approximate earlier methods. Given recent attempts to make the modern theory accessible, it might be time for all to enjoy modern thinking and the possibility of linking force computation to practical spectroscopy.

Here there is another tension between tradition and modern practice. Biologists and chemists typically think about optical properties in the visible and infrared regions that are useful for material characterization. As it happens, dispersion forces are often dominated by optical properties at deep UV frequencies. When difficulties in obtaining full spectral optical properties are encountered, the temptation has often been to give up and simply approximate or ignore what are evasively imagined to be "weak" interactions. Eliminating this barrier between solid electrodynamics and practice in other fields would liberate creative thinking.

Because of the speed, ease, and low cost by which information can be generated, $a b$ initio codes may provide provisional spectra. But at the atomic level, ab initio calculations are still limited to relatively small systems. The long range of the vdW-Ld interaction requires that many atoms be considered together. Large biological systems, such as interacting proteins and DNA and systems of like size found in many fields of physics and chemistry, still remain out of reach.

We need ways to handle metals and semimetals, graphene, and nanotubes. An important issue is that the nonretarded asymptotic forms differ from the integral inverse power laws in certain geometries of reduced dimensionality [see, e.g., Barash and Notysh (1988); Boström and Sernelius (2000); Dobson et al. (2006)]. The long range limits of the vdW interaction between infinite metallic fragments of reduced dimensionality have long range tails that decay more slowly than expected from $r^{-6}$ summation. Polarizabilities of fragments may become singular for a small frequency and wave vector. The possibilities are particularly intriguing with graphene layers, which can be weak metals in some of their forms.

A summary list of needs and opportunities working with electrodynamic forces includes the following: a common language between the continuum and atomistic communities; procedures to interpolate between the continuum and atomistic regimes, or at least to identify the distance limits when each formulation and its assumptions break down; efficient and reliable analytical and numerical methods for computing forces (and torques) between objects of arbitrary shape and material; accounting for anisotropy in material properties of nanotubes and other objects; alternative methods for electronic structure calculations incorporating dispersion forces; interaction potentials for complex biomolecules such as DNA; interactions of biopolymers with substrates; temperature or pressure dependence of force and associated Hamaker coefficients; wetting on patterned substrates and more complex geometries; thermodynamic "buoyancy" to account for the properties of complex media; dynamic Casimir phenomena such as dissipation between mutually moving plates; multibody and/or repulsive vdW-Ld effects for $a b$ initio codes; and vdW-Ld functionals for DFT codes that can coexist with methods for solving cavitation and solvation energies.

\section{B. Electrostatic interactions}

Not only for their inverse-square-power reach but also for their dielectric-breakdown strengths, the fields around the simplest ions frustrate us. Looking back to the triumphs of the 1920s that gave some idea of ion "activity" (Debye, Huckel) and self-energy (Born), we realize how little we have advanced beyond that pioneering decade. Work in the decade that followed (Onsager, Kirkwood, and Langmuir) taught us when and when not to use the simple ideas, while the 1940s and 1950s were a time when these ideas entered common practice with their limitations progressively forgotten.

Now, the founding ideas of ionic self-energies and electrostatic double layers permeate most current thinking so strongly as to trap us from learning precisely what we need to learn on the nanoscale where electric fields are strongest and where the structure of media dominate their dielectric response. The most facile common practice treats liquid water on the molecular scale as a continuum, little different from the dielectric material examined between the plates of a macroscopic capacitor; frequently imagines ions as featureless charges; and sometimes assumes that spatial averages are the same as time averages (Ben-Yaakov et al., 2009). There are clear 
exceptions of which we consider, but these exceptions too often tend to improve one feature of traditional pictures without concomitant correction of others. There do exist cases where continuum electrostatics can be a good approximation, for example, phenomena that are dominated by averages over many molecules.

As is usual, our best guide to properly formulate these additional features of electrostatic interactions on the nanolevel is the experiment itself. The direct measurements of interactions between charged macroions in ionic solutions, be it with the surface force apparatus (Israelachvili and Adams, 1978), the osmotic stress technique (Parsegian et al., 1986), or the atomic force microscopy (Munday and Capasso, 2007), provide us with the details of the measured electrostatic interactions that cannot be captured by the traditional point of view found in the classical literature (Verwey and Overbeek, 1948; Derjaguin, Churaev, and Miller, 1987; Evans and Wennerström, 1999; Israelachvili, 2006).

\section{Electrostatics in equilibrium statistical mechanics: Electrostatic double layers}

Much of the current understanding of electrostatics is based on mean-field Poisson-Boltzmann (PB) thinking. When the PB approximation does not agree with experiments it is still common to patch up the PB theory by introducing various devices rather than questioning its basis. There are cases where the PB approximation works, e.g., for dilute univalent aqueous electrolytes and weak fields far from charged surfaces (Hunter, 2001), but for other systems patched up PB approaches more often hinder rather than advance understanding. Where to look for next steps?

The most convenient place is theory itself. For example, effects of many-body correlations were surprising when they were initially found, but suggested possibilities of like-charge attractions (Oosawa, 1971; Guldbrand et al., 1984; Kjellander and Marcelja, 1984) and effective charge reversals (Lozada-Cassou et al., 1982; Valleau and Torrie, 1982; Outhwaite and Bhuiyan, 1983; Ennis et al., 1996). The thought is that ion-ion correlations in divalent and multivalent solutions contribute to these kinds of effects in real systems and sometimes cause them (even in the absence of specific ion adsorption) (Netz, 2001; Moreira and Netz, 2002). Nonzero effective charges for electroneutral particles, e.g., a hard sphere or surface in asymmetric electrolytes, is another example. These are cases where solution composition determines even qualitative features of the interactions.

The importance of ion-ion correlation effects between multivalent counterions for the appearance of attractions between equally charged particles has been recognized in many kinds of systems, although it is still taking a long time for this knowledge to spread (Boroudjerdi et al., 2005; Naji et al., 2005). Early examples include DNA (Guldbrand et al., 1986), lamellar surfactant phases (Wennerström et al., 1991), clay minerals (Kjellander et al., 1988) and mica surfaces (Kékicheff et al., 1993). An example of practical value is the cohesion of cement paste (Jönsson et al., 2005; Labbez et al., 2007).

Better than theory is the recognition of measured forces between charged materials. Thus we keep clearest in mind lessons from nature not from computers. To illustrate we focus on the properties of DNA in electrolyte solution as a model system.

\section{a. Model system: DNA-DNA interactions in charged electrolytes}

In the simplest limits, predictions of the PB theory for interactions between charged macroions in electrolyte solutions of univalent salts conform to osmotic stress measurements on ordered DNA arrays (Strey et al., 1998; Podgornik et al., 1998). With some adjustment for assumed charge density on the molecule, but partly compensated by bound ions, there is near quantitative agreement between theory and experiment (Strey et al., 1998). This agreement holds at distances greater than a Debye length between molecules. At closer separations, there are qualitative deviations from the simplest electrostatic double-layer theories of charged-rod repulsions. The sources of these deviations are likely due in part to the nonuniform charge of the molecule and to powerful solvation forces (Leikin et al., 1993). The latter can be described as structural forces or as structure-dependent dielectric response. The major feature is a failure of classical PB theory, a failure that occurs precisely in the important nanometer range that is the focus of our review and that is relevant to macromolecular assembly.

In salt solutions containing at least higher valence counterions, such as $\mathrm{Mn}^{2+}, \mathrm{Co}\left(\mathrm{NH}_{3}\right)_{6}{ }^{3+}$, or various polyamines, $\mathrm{PB}$ predictions lose all agreement with experiment. Not only does the PB theory give the wrong numerical values for the strength of the electrostatic interactions but also, and more importantly, misses their sign. Measurements point to the existence of attractions that do not follow mean-field electrostatic double-layer theory (Rau and Parsegian, 1992). This attraction is deduced from the shape of the osmotic pressure as a function of density of DNA as well as from direct magnetictweezer measurements of the attraction that condenses the double helices (Todd and Rau, 2008; Todd et al., 2008). The relevant measurements resemble pressure versus volume isotherms for gasses and liquids. Rather than attractive van der Waals interactions between gas molecules, for DNA the drive comes from the polyvalent counterion. For sufficiently large concentrations of $\mathrm{Co}\left(\mathrm{NH}_{3}\right)_{6}{ }^{3+}$, the DNA array spontaneously precipitates or condenses into an ordered high-density phase. One thus concludes that the polyvalent counterion confers attractions on nominally equally charged DNA molecules (Podgornik et al., 2008).

van der Waals interactions are much too small to account for the strong attractions seen with the addition of polyvalent counterions. Electrostatics on a mean-field level in cases of univalent counterions cannot give attractions (Andelman, 1995). In the presence of polyvalent counterions, one must go beyond the PB approximation and include the effects of ion-ion correlations as in simulations (Guldbrand et al., 1984), integral equation 
theory (Kjellander and Marcelja, 1984), or strongcoupling and strong-correlation approximations (Grosberg et al., 2002; Boroudjerdi et al., 2005; Naji et al., 2005). However, this may not be sufficient unless hydration effects and the details of the DNA structure are included. One way is to describe DNA attraction or repulsion at nanometer separations with an ad hoc orderparameter formalism built on perturbation of hydration forces (Leikin et al., 1993). There is clear deviation from the simplest electrostatic double-layer models routinely used in current theorizing. Inclusion of helical structure brings out some features that might explain certain facets of measurement as indicated by Kornyshev et al. (2007).

\section{b. Electrostatics with surfaces and interfaces}

Stabilization of colloidal dispersions has been observed to be due to the appearance of substantial effective charges for weakly charged macroparticles from correlation effects among smaller charged particles (Liu and Luijten, 2005; Martinez et al., 2005). Clustering and phase separation in systems with highly and equally charged colloidal particles can be created by counterioncounterion correlations (Hribar and Vlachy, 2000; Reščič and Linse, 2001; Linse, 2005; Hynninen and Panagiotopoulos, 2007). In some cases, various forms of depletion interactions can play a decisive role; they can for instance be caused by electrostatic ion-ion correlation or excluded-volume effects (Kanduc, Dobnikar, and Podgornik, 2009).

A common kind of depletion interaction (Verma et al., 1998; Roth et al., 2000) is an attraction between large colloidal particles that are in a suspension containing small colloidal particles, e.g., polymers (Schlesener et al., 2001) or disklike platelets (Harnau and Dietrich, 2004). The origin of this attraction is in the fact that small colloidal particles and large colloidal particles cannot overlap. Thus small colloidal particles are excluded from the depletion region in the vicinity of large colloidal particles. Furthermore, if the depletion regions of two large colloidal particles overlap, there is an osmotic pressure pushing the particles together, creating an effective attractive interaction potential (Triantafillou and Kamien, 1999). The range of the depletion interaction is given by the hard core radius of the small colloidal particles or equivalently by the polymer radius of gyration. One is thus in a position to vary the range (polymer molecular weight) or the strength (the polymer concentration) of an attractive depletion potential between large colloidal particles.

The study of polar liquids and electric double layers near one surface and interactions between two surfaces (e.g., double-layer interactions) are closely related to the study of properties of electrolytes and polar liquids in pores and other confined geometries. All are special cases of inhomogeneous fluids. In the presence of surfaces, electrostatic correlations, and thereby the electric screening, are profoundly changed compared to bulk electrolytes [power-law screening along a surface (Jancovici, 1982), exponential screening perpendicular to it].
The treatment of nonlocal dielectric response, dielectric saturation, and other effects on solvent due to electrostatic fields from surfaces, molecules, and other particles is a long-standing issue (Bopp et al., 1998; Yeh and Berkowitz, 1999; Ballenegger and Hansen, 2005; Ballenegger et al., 2006).

The complexity of electrostatic interactions and their coupling to other modes of interactions is illustrated by the interdependence of dispersion and electrostatic interactions (Ninham and Yaminsky, 1997; Kunz et al., 2004; Tavares et al., 2004; Wernersson and Kjellander, 2007). Ion-macromolecule dispersion interactions can explain some of the ion specificity seen in effects of electrolytes on macromolecular interactions. vdW-Ld interactions between the constituent molecules of electrolytes affect the screening of electrostatic interactions in nontrivial ways, for instance, by changing the exponential screening in bulk to a power-law screening in both quantum (Brydges and Martin, 1999) and classical (Kjellander and Forsberg, 2005) statistical mechanics theory. Especially with the added consideration of related interactions, such as ion-solvent induced-dipole interactions, more attention should be spent on the correct treatment of the screening by electrolytes of the static part of the van der Waals interaction (Mahanty and Ninham, 1976; Parsegian, 2005), which is relevant in some systems like aqueous electrolytes common in biology. Likewise, other static polarization effects like the ion-solvent induceddipole interactions are also screened in electrolytes, while the high-frequency part of the dispersion interaction is not.

It was recently realized that in electrolytes the anisotropy of the electrostatic potential from a molecule extends to the far field region (Rowan et al., 2000; Hoffmann et al., 2004). The full directional dependence of the electrostatic potential from a charged or uncharged molecule in electrolytes remains in the longest range tail (i.e., from all multipole moments) (Ramirez and Kjellander, 2006). In particular, the range of the potential from an ion and that from an electroneutral polar particle is the same. This is contrary to the case in vacuum or pure polar liquids, where the potential from a single charge is longer ranged than that from a dipole, which in turn is more long ranged than from a quadrupole, etc. The orientational dependence of the electrostatic interaction between two molecules in electrolytes is therefore complex even at large distances, and the consequences of this complexity must be further explored (Trizac et al., 2002; Agra et al., 2004).

\section{Electrostatics in density functional theory}

Following the Born-Oppenheimer approximation, we refer here to all properties of matter for which the electrons may be treated as in their ground state. Nuclei or ions may be treated as classical particles in whose potential the electrons move. In this approximation then ions are in no sense in their ground state and can redistribute in response to gradients in temperature, stress, electric fields, chemical potential, etc. 
The range of validity of the Born-Oppenheimer approximation includes most of the chemistry as well as equilibrium and nonequilibrium thermodynamics of materials and much of biology. It includes all interatomic forces between ions, thermally activated atoms, in electrolytes, metals, insulators and semiconductors, van der Waals solids, liquids and gases, while excluding fastmoving ions in radiation damage events, radiative processes, such as luminescence, and electron transport in which quantum electrodynamics or electromagnetism start to play a role. It matches the range of validity of density functional theory (Hohenberg and Kohn, 1964; Kohn and Sham, 1965), which is a unifying principle, the basis of many practical schemes of calculation, and a source of insights into the long range electrostatic interactions (Finnis, 2003).

The LDA or GGA paradigms have led to codes that are fast compared to any tractable quantum chemical techniques that might be more accurate, such as those involving multiconfiguration wave functions, or quantum Monte Carlo. Nevertheless, it should be recognized that the inherent absolute errors in standard DFT or LDA may be of order $0.1 \mathrm{eV}(\sim 1200 \mathrm{~K})$ per bond or more in some cases, which is too large for many questions in chemistry and particularly biology.

\section{Challenges and opportunities}

As with well-established vdW-Ld formulations, there is sometimes a perception that there is little else fundamental to discover in electrostatics and that at least by computer simulation we can solve every system of interest. Although the present practice of electrostatics rests on some solid long-standing foundations, both the quantum mechanical and classical statistical mechanical aspects require enormous improvement.

Within its framework, density functional theory already provides a systematic way to obtain all kinds of all LRIs in condensed materials. Besides the difficulty of applying the procedure, it requires that the BornOppenheimer approximation be valid and that electrons be close to their ground state. The recent introduction of nonlocal functionals by Langreth and co-workers (see Sec. II.A.4.a) promised to extend the practical scope of DFT to situations in which dispersion forces are important, by including nonlocal contributions to correlation energy. At least for some systems this might give a useful approximation to the dispersion interaction and supersede the addition of two-body potentials. However, there are significant impediments to progress. In metals, long ranged electrostatic forces manifest themselves as anomalies in phonon spectra, and even in elastic moduli. Charged point defects in insulators require long length and time scales to simulate the equilibrium distributions, together with the detailed electronic structure calculations needed to obtain their formation and segregation energies.

In the domain of electrostatic double layers and charge interactions in general, with mobile ions whose distributions depend on the very potentials they them- selves create, the situation is daunting. Particularly on the nanometer scale, when continuum electrostatics is inappropriate, the need to incorporate solvent structure as well as ionic personality immediately removes us from the domain of traditional theories. There is a need to look at real experimental data that probe situations of ignorance. Elaborate simulations alone do not suffice. Simulations need to be validated against measured forces before they can be trusted to teach us where direct experimental data do not exist.

A significant body of data already exists on directly measured forces over the few nanometers approaching molecular contact. The extent to which the simplest theories must be recast to recognize molecular structure and structural forces is still completely open. Without accurate representation of measured forces, simulations are in danger of being hypothetical exercises. One can obtain exact statistical mechanical results in certain limits (e.g., low densities, high or low temperatures, or large distances). Such limits do provide estimates from which important aspects of the systems might be recognized (Boroudjerdi et al., 2005; Naji et al., 2005).

Theoretical problems for nanoscale systems often involve several simultaneously important length scales. One strategy might be to integrate simulations with results from formal theory in ways that enhance applicability of simulations. For example, there exist formal results for the long range tails of various distribution functions that rely on the behavior at short range (González-Mozuelos and Bagatella-Flores, 2000; Kjellander and Ramirez, 2005). There have been attempts to construct effective interaction potentials where some molecular degrees of freedom are included implicitly (Lyubartsev and Laaksonen, 1995, 2004; Ayton et al., 2007; Tóth, 2007). The idea is to treat several length scales at the same time; for example, the small size of the solvent molecules and dissolved salt ions, the appreciably larger size of the macroparticles or aggregates, and the average separation between particles (Lobaskin et al., 2001). Even when the main interest concerns phenomena on the large scale, the effects of the molecular details somehow have to be taken into account without losing their important features. "First principles" simulations are still far too expensive and idealized to treat most problems in soft matter science, even for simple aqueous systems [see Schwegler (2007)].

Ideally, one should first eliminate the possibility that the deviations, too often dismissed, occur because the theory used is too approximate. One example of this is the PB treatment of primitive model electrolyte systems. When the true properties of the primitive model were evaluated with accurate statistical mechanical methods, many new features were found beyond PB approximation (Ben-Yaakov et al., 2009; Kanduc, Naji, Jho, Pincus, and Podgornik, 2009). Several of the observed deviations between experiments and the PB prediction were due to the mathematical approximations implicit in the PB approach and not due to the underlying model. Even worse, comparisons of any model with measurements continue to be avoided. Until measurements are given 
precedence over models, our idealized pictures of electrostatics forces will continue to cripple productive thinking.

\section{Polar interactions}

\section{Motivation and recent advances}

Although not as long ranged and clearly defined as electrostatic and vdW-Ld interactions, polar [or sometimes referred to as acid-base $(\mathrm{AB})]$ interactions play important roles in, for example, chemical reaction, adhesion, triboelectrification, and colloidal interactions. There are several ways to speak of them: donor-acceptor interactions (Gutmann, 1978), hard-soft AB (Parr and Pearson, 1983), and through the equations of Drago and Wayland (1965). What can be learned from these various theories is that a polar, or $\mathrm{AB}$, interaction is composed of Coulombic, covalent, and charge-transfer interactions.

The first two (electrostatic and covalent) are often approximately expressed as (Hudson and Klopman, 1967)

$$
\Delta E=-\frac{q_{1} q_{2}}{R_{12} \varepsilon}+2 \sum_{m} \sum_{n}\left[\frac{\left(c_{1}^{m}\right)^{2}\left(c_{2}^{n}\right)^{2} \beta^{2}}{E_{m}^{*}-E_{n}^{*}}\right] .
$$

The first term is an idealized Coulombic interaction; and the second term refers to outer orbital interactions. In Eq. (11), $m$ and $n$ denote the donor and acceptor orbitals; $c_{1}^{m}$ and $c_{2}^{n}$ are the coefficients of atomic orbitals participating in interaction; and $\beta$ is a resonance integral. $E_{m}^{*}$ and $E_{n}^{*}$ are the energy of the donor and acceptor orbitals, equivalent to the energies of their highest occupied molecular orbital (HOMO) and the lowest unoccupied molecular orbital (LUMO). Drago's empirical version of Eq. (11) describes the heat of formation $(-\Delta H)$ of $A B$ complex:

$$
-\Delta H=E_{A} E_{B}+C_{A} C_{B},
$$

$E$ and $C$ are the electrostatic and covalent interaction constants, respectively. Drago's equation was used by Fowkes (1963) to study the effects of $A B$ interactions on wetting, adsorption, and adhesion.

Among their many idealizations, these ancient relations, Eqs. (11) and (12), do not recognize the important charge-transfer interaction for which Parr and Pearsons's HSAB (hard-soft $A B$ ) principle has been used with two important parameters: absolute electronegativity $(\chi)$ and absolute hardness $(\eta)$. Using density functional theory, Parr and Pearson showed that the absolute electronegativity is a chemical potential of electrons, and hardness is the derivative of this chemical potential with respect to the number of electrons. Formally,

$$
\chi=-\left[\frac{\partial E}{\partial N}\right]_{Z}=\frac{1}{2}(I+A)
$$

and

$$
\eta=\frac{1}{2}\left[\frac{\partial^{2} E}{\partial N^{2}}\right]_{Z}=\frac{1}{2}(I-A) .
$$

$I$ is the ionization potential and $A$ is the electron affinity of a species. Parr and Pearson also estimated the change $\Delta E$ of the electronic energy associated with charge transfer from a donor to an acceptor as

$$
\Delta E=-\frac{\left(\chi_{A}^{0}-\chi_{B}^{0}\right)^{2}}{4\left(\eta_{A}+\eta_{B}\right)}
$$

Because the softness of a species is a chemical potential of electrons, $\chi^{0}$ corresponds to the standard chemical state. According to the HSAB principle, strong $A B$ interactions result when the gap between the HOMO of the donor and the LUMO of the acceptor is very low, leading to a "soft" $A B$ interaction. If the above gap is large, there will be little $A B$ interaction via chargetransfer complexation so that the primary $A B$ interaction is due to electrostatics.

According to Derjaguin et al. (1973), as electrons are transferred across an interface from the donor to the acceptor sites, an electric potential difference $[\Delta V(n)]$ develops depending upon the numbers of electrons transferred, or the $A B$ pairs $(n)$ formed, across the interface. By minimizing the total free energy of the system with respect to $n$,

$$
\frac{n}{N-n}=\exp \left[\frac{-\Delta E-e \Delta V(n)-e n \partial V(n) / \partial n}{k T}\right] .
$$

Treated as an experimentally determined parameter, $\Delta E$ should be exactly the same as in Eq. (15). Another equation relating $\Delta V(n)$ and $n$ is, however, necessary to obtain the optimum number of $A B$ pair and the resulting electrical potential across an interface. Equation (15) in conjunction with Eq. (16) form the basis for a wellknown phenomenon - triboelectrification-on the basis of $A B$ interaction across an interface. Fowkes (1953) first proposed that the interfacial interaction can be decomposed into two terms, one arising from the dispersion forces and the other arising from the $A B$ interaction. The treatment of Van Oss et al. $(1987,1988)$ is similar to Fowkes in that the interfacial energy is expressed as $\gamma^{\mathrm{TOT}}=\gamma^{L W}+\gamma^{A B}$. While the vdW-Ld component of the adhesive interaction follows the geometric combining rule (i.e., $W_{12}^{L W}=2 \sqrt{\gamma_{1}^{L W} \gamma_{2}^{L W}}$ ), the $A B$ component does not. The $A B$ component of the adhesive interaction is expressed as

$$
W_{12}^{A B}=2 \sqrt{\gamma_{1}^{+} \gamma_{2}^{-}}+2 \sqrt{\gamma_{1}^{-} \gamma_{2}^{+}} .
$$

The geometric-mean combining rule used is a good approximation for describing surface properties of media where nonpolar interactions dominate and is based on the assumption that the principal dielectric absorption frequencies of the media are similar (Israelachvili, 2006).

Thus, the $A B$ component of interfacial energy $\gamma^{A B}$ comprises two nonadditive parameters, an electronacceptor surface tension parameter $\left(\gamma^{+}\right)$and an electrondonor surface tension parameter $\left(\gamma^{-}\right)$. The total $A B$ con- 


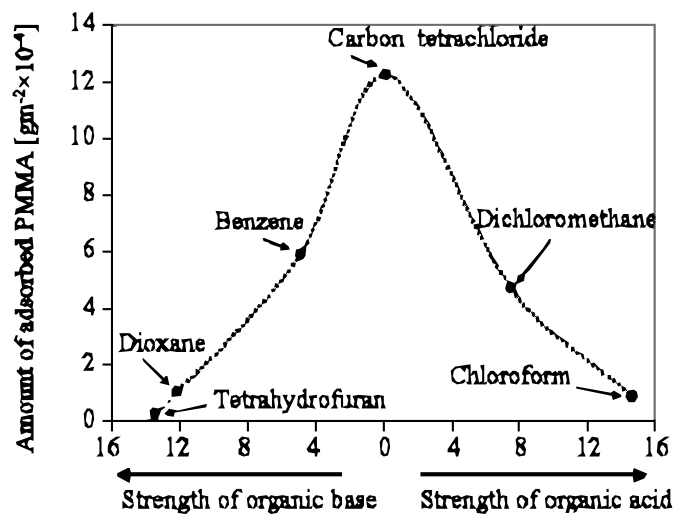

FIG. 5. Role of acid-base interaction in polymer adsorption. The adsorption of a basic polymer, poly-methymethacrylate (PMMA), onto an acidic silica is maximal when the adsorption occurs in a neutral solvent, carbon tetrachloride. However, when the adsorption occurs in either acidic or basic solvent, adsorption is reduced as the solvent interacts either with PMMA or silica. From Fowkes, 1983.

tribution to the surface tension is given by $\gamma^{A B}$ $=2 \sqrt{\gamma^{+} \gamma^{-}}$. The total interfacial tension between condensed phases $i$ and $j$ is described by

$$
\gamma_{i j}^{A B}=2\left(\sqrt{\gamma_{i}^{+} \gamma_{i}^{-}}+\sqrt{\gamma_{j}^{+} \gamma_{j}^{-}}-\sqrt{\gamma_{i}^{+} \gamma_{j}^{-}}-\sqrt{\gamma_{i}^{-} \gamma_{j}^{+}}\right)
$$

which is not a geometric combining rule, but rather expresses the doubly asymmetric interaction between two different materials resulting from the fact that a material can be a good electron donor, electron acceptor, neither (apolar), or both (bipolar). This theory predicts that monopolar (predominantly acidic or basic) materials will strongly interact with bipolar materials or with monopolar materials of the opposite type, and if this adhesive interaction is strong enough, the interfacial interaction between two condensed phases can become negative. Based on the Dupré equation, which can be applied for both polar and nonpolar materials, the polar interactions between two solid materials 1 and 2 in a liquid medium 3 using the interfacial energy given by Eq. (4), is

$$
\begin{aligned}
\Delta G_{132}^{A B}= & \gamma_{12}-\gamma_{13}-\gamma_{23} \\
= & 2\left[\left(\sqrt{\gamma_{1}^{+}}-\sqrt{\gamma_{2}^{+}}\right)\left(\sqrt{\overline{\gamma_{1}^{-}}}-\sqrt{\gamma_{2}^{-}}\right)\right. \\
& -\left(\sqrt{\gamma_{1}^{+}}-\sqrt{\gamma_{3}^{+}}\right)\left(\sqrt{\overline{\gamma_{1}^{-}}}-\sqrt{\gamma_{3}^{-}}\right) \\
& \left.-\left(\sqrt{\overline{\gamma_{2}^{+}}}-\sqrt{\gamma_{3}^{+}}\right)\left(\sqrt{\overline{\gamma_{2}^{-}}}-\sqrt{\overline{\gamma_{3}^{-}}}\right)\right] .
\end{aligned}
$$

For two identical or different polar materials separated by a solvent, it is then possible for the interaction to be repulsive or attractive.

These concepts have been applied to a variety of phenomena in condensed phases. In numerous liquids and polymers, the quantitative interpretation of surface tensions is incomplete without inclusion of $A B$ interaction energies as shown in Fig. 5. The same is true of the solubility of various polymers in solvents. $A B$ interactions can also generate osmotic pressure more than a hundredfold greater than that due to van't Hoff. The $A B$ approach can give clear indications about the nature of

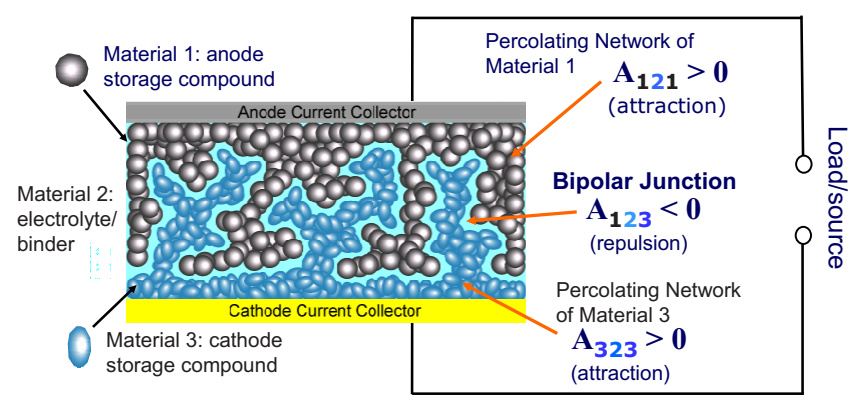

FIG. 6. (Color online) Colloidal-scale self-organizing lithiumion battery concept, demonstrated in graphite- $\mathrm{LiCoO}_{2}$ system, making use of acid or base forces for junction formation and particle assembly.

complex surfaces, for example, the preferential segregation of certain groups on the surface of solid copolymers (Adão et al., 1999). Parameters have also been determined for biological surfaces such as skin (Mavon et al., 1998) and bacterial cells (Ong et al., 1999). Empirical methods for estimating the strength of $\mathrm{AB}$ interactions are summarized by Chaudhury (1996). There nonetheless remains a lack of consensus regarding the most appropriate approach to quantification of the strength of the $A B$ interaction (Correia et al., 1997; Douillard, 1997; Lee, 1998).

$A B$ interactions are therefore important as part of the LRIs "tool kit" for understanding physical behavior, and, in the future, for the manipulation and design of new materials and devices. One recent example concerns the use of $A B$ interactions to produce selforganized devices such as lithium-ion batteries (Fig. 6). Cho et al. (2007) proposed a general approach to the direct formation of bipolar devices from heterogeneous colloids in which attractive and repulsive interactions could be combined to produce a network of one material (e.g., an anode) that is everywhere separated from a network of a second (e.g., a cathode) An ensuing search for suitable combinations of conductive device materials and solvents using atomic force microscopy (AFM) measurements showed first that inclusion of $A B$ interactions was essential to understanding experimental data for the inorganic compounds studied, and second led to the successful identification of several electronically conductive materials [carbon, indium tin oxide (ITO), $\mathrm{LiCoO}_{2}$ ] between which repulsive $A B$ interactions are obtained in an appropriately chosen liquid medium and in the absence of electrostatics. As a result, a colloidal-scale selforganized lithium rechargeable battery based on graphite- $\mathrm{LiCoO}_{2}$ was demonstrated (Fig. 6).

\section{Challenges and opportunities}

Given the vintage of many ideas still being used as well as limited experimental information to allow practical application, it is clear that this subject needs reformulation and quantitative measurement. There have been few studies attempting to generalize the methods for estimating $A B$ interactions based on molecular con- 

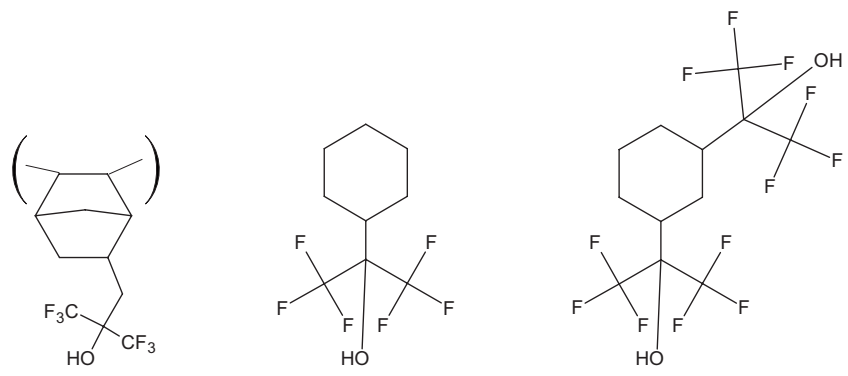

FIG. 7. Examples of fluoropolymers which may serve as a $\gamma+$-reference solid to be used for the analysis of monopolar surfaces and liquids interactions.

cepts of hardness and softness in acids and bases to other classes of materials such as metals and semiconductors (Cain et al., 1969; Ho et al., 1991). While the current theories of $A B$ interactions can reasonably predict whether a specific $A B$ interaction will prevail, the ability to predict quantitatively the strength of $A B$ interaction is poor. Our belief is that they remain conceptually important.

Experimentally, few data exist for classes of materials besides the most simple liquids and polymers. A major difference from Hamaker coefficients is that a systematic cataloging of more coefficients is straightforward compared with similar systematic cataloging of $A B$ interactions. This is an area ripe for scientific advance.

While $A B$ interactions have the potential to be a key tool in material and device design, improved fundamental understanding is essential for their use in the synthesis of materials as well as in the production of selforganizing junctions, subassemblies, devices, and other applications. Metals, semiconductors, insulators, and biological materials are all likely components of such "engineered" devices.

So far, only indirect methods such as wetting and solubility have been used to characterize $A B$ interactions. It is time that more modern methods be used to measure interactions directly and at nanoscale spatial resolution, methods such as scanning probes and force microscopy with chemically modified tips.

Many more measurements are needed to connect polar behavior with fundamental properties. There are few monopolar materials of the electron-acceptor type (Lee and Sigmund, 2002). One important class of such compounds may be fluoroalcohols and fluoroalcohol-bearing polymers (see Fig. 7). These materials have been shown experimentally to be hydrogen bond donors only, with no capability to act as hydrogen acceptors. It may be that $a b$ initio codes can enhance or extend needed measurement. If first-principles calculations were able to agree with currently available experimental results, it might be possible to enlarge the list of wellcharacterized materials. This enlargement would be useful for chemists, biologists, and others for material selection and experimental design purposes.

\section{INSTRUCTIVE SYSTEMS}

Having identified fundamental forces, how do we now learn about them? In Sec. III.A, we discuss computation of optical spectra and Hamaker coefficients of complex biomolecular systems including single-wall carbon nanotubes (SWCNT) and B-DNA. We then consider several examples of macromolecules and polyelectrolytes: anionic-cationic polyelectrolyte complexes, hydration interactions with ionic specificity, extraction or separation in phase transfer reactions, and surfactant-decorated interfaces. Impurity-based quasiliquid films and space charges at solid interfaces are discussed in Sec. III.B; these two phenomena often intermingle at the grain boundaries and surfaces of many structural and functional ceramics. Section III.C presents instructive systems related to aqueous solutions and suspensions: premelting and related phenomena in ice and water, ion hydration, oxide/electrolyte interfaces, colloidal suspensions, and SWCNT hybrids. Further aspects are deferred to Sec. IV for practical implications and technological importance.

\section{A. Atoms and molecules}

\section{Optical spectra and Lifshitz theory for complex biomolecular systems}

For systems of high complexity and fragility, such as biomolecular membranes or proteins, experimental measurement of optical spectra using vacuum ultraviolet spectroscopy is not yet possible. Although theoretical calculations of optical spectra for such systems are daunting, new computational methodologies and theory finally make such calculations feasible. Calculation of optical properties via $a b$ initio theories has been discussed in Sec. II.A.3. Here we present the example of SWCNTs.

As demonstrated for ceramic crystals (Ahuja et al., 2004) and SWCNTs (Rajter et al., 2008), calculation of optical properties at the level of local density approximation (LDA) of the density functional theory (DFT) in the random phase approximation (RPA) seems to be adequate. Higher-level theory, such as time-dependent density functional theory (TDDFT), or theories that include some aspects of many-body corrections or selfinteraction correction (SIC) might be sometimes necessary.

In addition to LDA, DFT, and RPA, it is necessary to make further approximations. First, for geometrical shapes of real objects, in order to use available analytic formulas one might describe a small-radius SWCNT as a cylinder, a bucky ball as a sphere, graphene as an infinite planar sheet, or biomembranes as plane-parallel blocks. Second, in real situations where several media are involved, there are additional approximations for averaging or mixing the dielectric functions (Brosseau, 2006). Third, practical calculation of optical dielectric functions for large complex biological systems is still computationally prohibitive. Further developments of computational 


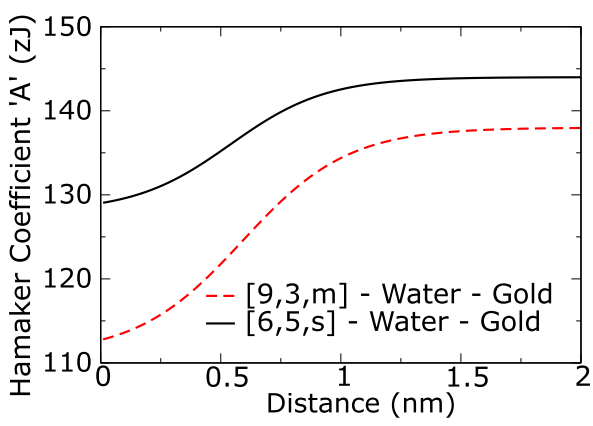

FIG. 8. (Color online) The Hamaker coefficients $(A)$ of SWCNTs. For $[9,3, m]$ and $[6,5, s]$ SWCNTs in water as a function of $l$ for gold substrate. From Rajter et al., 2007.

codes, identified criteria of accuracy, and new computational resources are necessary.

\section{a. Optical properties of SWCNTS}

Computed Hamaker coefficients for two types of CNTs with gold in a water medium are shown in Fig. 8. First, look at the changes in the Hamaker coefficient versus separation along the radial direction of the SWCNT-water-Au substrate system (Rajter et al., 2007). This calculation demonstrates the relative magnitude differences that occur because small changes in the chirality of the SWCNTs create large changes in optical spectra. It also highlights the importance of access to the full spectrum. Glancing at the vdW-Ld spectra curves in Sec. II.A.3, it might seem that the large low-energy wing in the $[9,3, m]$ spectrum would make the interaction much larger than for the semiconducting $[6,5, s]$. However, the $[6,5, s]$ has stronger interactions in the remainder of the Matsubara summation in both the near- and far-separation formulations, to create a stronger overall vdW-Ld interaction (Fig. 8).

The orientation dependence of the Hamaker coefficient itself is seen with anisotropic materials. In the far limit of the anisotropic cylinder-cylinder interaction, we see an increase of the Hamaker coefficient by a factor of nearly $30 \%$. Similar results were reported for the $\mathrm{Al}_{2} \mathrm{O}_{3}$ substrate-substrate system by Knowles (2005), although to a lesser extent because of a smaller degree of anisotropy. The potential implications for design and manipulation of pieces during construction of nanodevices are real.

As we incorporate the effects of a changing internal medium of the SWCNT core, and adding the effects of surfactant layers, etc., additional effects will likely become clear.

\section{b. Optical properties of B-DNA}

$A b$ initio optical properties of B-DNA and collagen have been obtained using the DFT-based orthogonalized linear combination of atomic orbitals (OLCAO) method (Ching, 1990). In these calculations, $\mathrm{Na}$ ions were added to the bare DNA model to neutralize the negatively charged $\mathrm{PO}_{4}$ groups from the DNA backbone. Without the compensating counter ions, the self-consistent po-

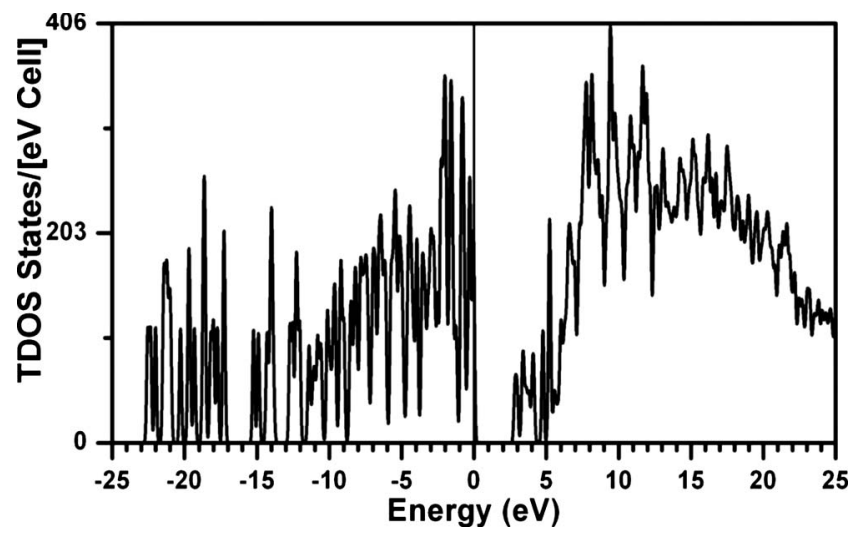

FIG. 9. The calculated total density of states (TDOS) of a periodic B-DNA model with $10 \mathrm{CG}$ base pairs and with $20 \mathrm{Na}$ ions as counter ions added. The calculation shows this model has an insulating gap of about $2.0 \mathrm{eV}$.

tential in the electronic structure calculation will not converge. One such calculation is a periodic model (in the $z$ direction) with $10 \mathrm{CG}$ base pairs and $20 \mathrm{Na}$ counterions for b-DNA. This model contains a total of 650 atoms and 2220 valence electrons. The ab initio calculation shows it to be an insulator with a band gap of about $2.5 \mathrm{eV}$. Figures 9 and 10 show the calculated total density of states (TDOS) and the imaginary part of the frequency-dependent dielectric function $\varepsilon^{\prime \prime}(\omega)$ of the model (Rulis, Liang, and Ching, 2009).

These calculations use several advantages of the OLCAO method. First, the local orbital basis expansion keeps the total dimension of the Kohn-Sham equation at a manageable level for more than thousands of atoms. Second, the effective Gaussian representation in both the basis function and atom-centered potential functions facilitates the evaluation of multicenter integrals. Third, the inclusion of optical matrix elements in the calculation for transitions up to high-unoccupied states provides the needed accuracy at both the low and highfrequency limits. Finally, the ability to explore the interatomic, intermolecular, and intramolecular bonding using the concept of partial density of states (PDOS) from different groups of atoms facilitates the interpreta-

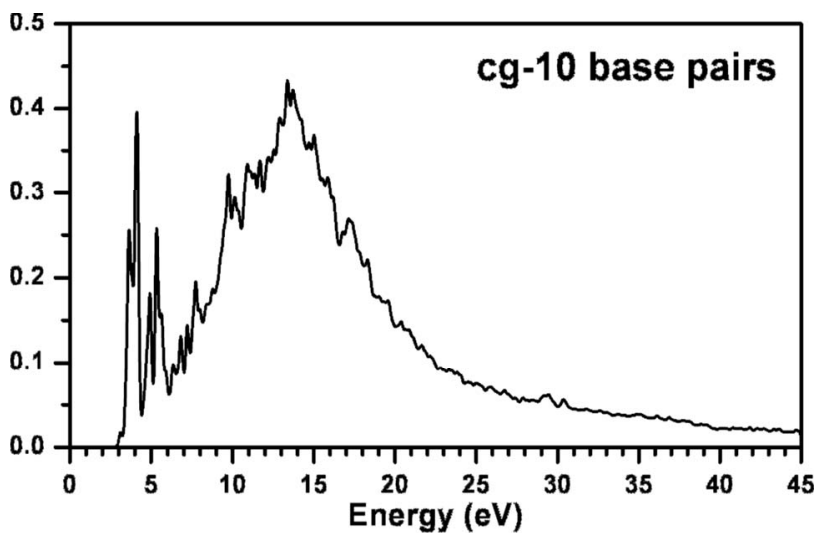

FIG. 10. The calculated imaginary part of the dielectric function of the b-DNA model of Fig. 9. 
tion of the calculated results. How such features affect force computation is as yet undetermined.

It is hoped that the same approach can be used to calculate optical spectra in other systems such as colloidal suspensions, intergranular films (IGFs) in polycrystalline ceramics (Secs. III.B.1 and IV.A.2), and interfacial models of different materials, which then can be used for quantitative estimation of the long range dispersion forces. We still have no reliable strategy how to include fluids and counter ions in the calculation of optical properties for force computation. On a larger scale, there is a critical need for realistic atomic-scale structural models for large biological molecules or complex microstructures in ceramics that can be used for $a b$ initio electronic and optical properties calculations.

\section{Hydration interaction and ionic specificity}

We focus here on aspects of phase stability of surfactant solutions or colloidal microcrystals when stability, coexistence, or swelling is due to a hydration force, and is not of immediate electrostatic origin. In such situations, the absence of an identified "electrostatic effect" such as a link between Debye lengths and phase limits is due either to the absence of charge or to an effect independent of the presence of added salt (Hao and Zemb, 2007). It may seem paradoxical to attribute long range to the hydration force, which can only persist for a length where the drive for structural alignment of the solvent around the solute can overcome the effects of Brownian motion (Todd et al., 2007). This force is "long" versus hydrogen bonding, complexation, and other nearestneighbor interactions considered in the chemistry of colloids. For good model systems, in the absence of salt, the hydration force can be detected by applied osmotic pressure as low as a few hundred $\mathrm{Pa}$ with typical distances between surfactant aggregates of up to $\sim 3 \mathrm{~nm}$ (Carriere et al., 2007). At $\sim 1 \mathrm{~nm}$, the hydration pressure can grow to hundreds of atmospheres between planar surfaces. Compared with the ultralong range of electrostatics in the absence of screening, the hydration force is short range. A combination of hydration force and electrostatics is the source of several behaviors of surfactants systems that can be explained only if hydration is considered as a fundamental repulsive mechanism which can dominate even in the absence of structural net charge: measurements mixing anionic and cationic components, producing colloidal aggregates of known charge, play an instructive role here (Ricoul et al., 1998).

The distance dependence characterizing exclusion of small solutes from macromolecular surfaces follows the same exponential behavior as the hydration force between macromolecules at close spacings. Similar repulsive forces are seen for the exclusion of nonpolar alcohols from highly charged DNA and of salts and small polar solutes from hydrophobically modified cellulose (Bonnet-Gonnet et al., 2001). Exclusion magnitudes for different salts follow the Hofmeister series that has long been thought connected with water structuring (Todd $e t$ al., 2008).
One feature is the intriguing connection with the distributions of salts in thin liquid films on ice. The connection between hydration effects in water and the Bjerrum defect distribution in ice has been noted before (Gruen and Marcelja, 1983) and is due to the structuring of water molecules close to macroscopic surfaces. In ice this is described by a redistribution of orientational Bjerrum defects, whereas in water it is usually discussed within water solvation or hydration models. In both cases, however, ion redistribution couples with hydration patterns.

Solvation of interacting macromolecular surfaces and modulation of this solvation by cosolutes such as salts exquisitely regulates equilibria of specific association in chemistry and biology. Depending on whether the cosolute is preferentially excluded from, or attracted to, the surfaces of the macromolecules, a cosolute can either increase or decrease complex stability (Harries and Parsegian, 2004). However, the dynamic action of cosolute on complexation is not yet understood, and there is no way to predict which kinetic constant, the "on rate" or the "off rate," has the greater impact.

A decade ago "molecular Coulter counting" demonstrated that a single protein nanopore could be used to detect polymer exchange between pore and bulk, a demonstration that stimulated modern development of nanosensors (Bezrukov et al., 1994). This same method also allows one to address the dynamic side of preferential solvation. Using an alpha-hemolysin nanopore as a sensor, it is possible to follow the effect of solutes on a simple complexation reaction at the single-molecule level (Gu et al., 1999). Monitoring transient obstruction of current through a nanopore complex reveals the kinetics underlying the reaction equilibrium in the presence of various cosolute salts. Measurements with alphahemolysin progressively blocked by cyclodextrin and adamantane reveal changes in on rates as well as off rates, depending on the type of salt used (Gurnev et al., 2009). Chloride and bromide salts mainly impact the off rates; sulfate changes the on rate, revealing qualitatively different dynamic action of different cosolutes (Harries and Rosgen, 2008).

\section{Extraction, separation, and phase transfer reactions}

Liquid-liquid phase transfer of metals in the form of ions is a crucial step in re-processing nuclear fuel. Currently available technologies rely on liquid-liquid extraction.

The existence and localization of the critical point position in the phase diagram of the extracting media have demonstrated the predominance of $\mathrm{vdW}$ forces, a long range interaction in phase separation of a dispersion of water nanodroplets covered by extracting molecules (Nave et al., 2004).

The extracting process involves the cations, associated anions, and co-extracted water. All these species are confined in a dehydrated form in the polar core of formed micelles. Using a series of homologous acids, it has been proven that the Hofmeister series (Collins, 2004) of the anion involved in the co-extraction process 


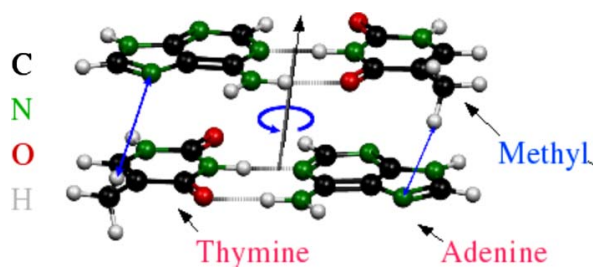

FIG. 11. (Color online) A typical stacking configuration used in this study. The two base pairs are attracted by the vdW-Ld interaction. In the lowest energy configuration they undergo a helical twist as shown, which reduces the Pauli repulsion. This is the so-called AT:AT step, where the first AT labels the nucleobases going up one strand while the second labels those going down the other.

profoundly influences the efficiency and selectivity of the extraction as well as the overall stability of the dispersion of reverse micelles involved in the process (Testard et al., 2007).

\section{DFT results on DNA base-pair vdW interactions}

In Sec. II.A.4, we reviewed a number of recently developed methods for including the vdW-Ld interaction in electronic DFT. Prior to these developments, DFT had been generally successful in the description of dense matter and isolated molecules. The newer methods are beginning to extend this success to sparse matter, biological matter, and vdW molecular complexes, and thereby to systems where DFT with conventional functionals generally have failed. Some of these methods are highly empirical, while others are not. Here we give an example of using nonempirical $\mathrm{vdW}$ density functional (vdW-DF) (Dion et al., 2004; Thonhauser et al., 2007) as applied to nucleic acid-base-pair steps (Cooper et al., 2008).

The latter work considers Watson-Crick base pairs in a stacking geometry as shown in Fig. 11. The work uses vdW-DF to calculate the interaction energy between the two components of each of the ten possible DNA basepair duplexes as a function of the so-called twist angle (see Fig. 11) with the separation (rise) between each component being optimized. In this way one could determine whether the twist and each step of a DNA polymer has its precursor within the properties of the isolated duplex step. By comparing the results with analyses (Olson et al., 2001) of high-resolution crystalline data from the nucleic acid database, they imply that on a broad scale the answer to this question is yes. They find a mean twist of $34^{\circ} \pm 10^{\circ}$, where the \pm sign indicates the standard deviation arising from sequence dependence. The Olson experimental database indicates a corresponding twist of $36^{\circ} \pm 7^{\circ}$.

More detailed results from Cooper et al. (2008) are shown in Fig. 12 for three steps which show revealing behavior. Simply stated base pairs gain on the order of 10 or more $\mathrm{kcal} / \mathrm{mol}$ by stacking in an untwisted configuration, and typically gain several more $\mathrm{kcal} / \mathrm{mol}$ by twisting by an amount of the same order of magnitude as found in high-resolution studies of crystalline DNA. As

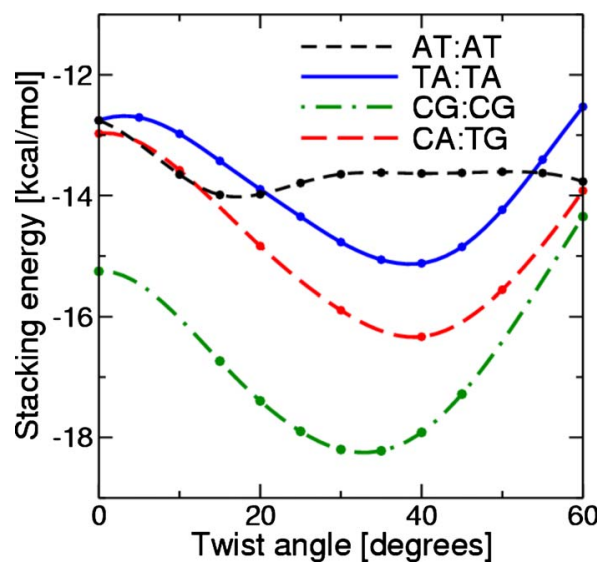

FIG. 12. (Color online) Stacking energy vs twist angle for the indicated base-pair steps, labeled as described in Fig. 11. The TA:TA, CG:CG, and CA:TG curves are typical. The AT:AT one is not; its kink at about $20^{\circ}$ was attributed to the hydrogennitrogen interaction indicated by the double-arrowed lines in Fig. 11.

for the sequence dependence of the twist, Cooper et al. pointed out that their results generally follow the trend variations of the databases, but with larger fluctuations.

From the above one can conclude that vdW-DF is sufficiently accurate to obtain meaningful results for this type of problem. Combining this information with the fact that the scaling with system size for vdW-DF is no worse than for standard DFT suggests that vdW-DF can be applied to systems that are significantly larger than those considered above, with reasonable confidence of obtaining valid predictions.

\section{B. Interfaces, surfaces, and defects in solids}

\section{Impurity-based quasiliquid surficial and interfacial films}

Nanoscale, impurity-based, quasiliquid interfacial films of similar character have been found in an increasing number of different material systems and configurations [Fig. 13; see Luo (2007), and references therein]. These include silicate-based IGFs in $\mathrm{Si}_{3} \mathrm{~N}_{4}, \mathrm{SiC}$, and several oxides (where $\mathrm{SiO}_{2}$ additive was once considered essential to stabilize such nanoscale IGFs); IGFs in $\mathrm{ZnO}-\mathrm{Bi}_{2} \mathrm{O}_{3}$ and $(\mathrm{Sr}, \mathrm{Ba}) \mathrm{TiO}_{3}$, where $\mathrm{SiO}_{2}$ is not involved; IGFs at oxide-oxide heterointerfaces; $\mathrm{SiO}_{2}$-enriched IGFs at metal-oxide interfaces; analogous IGFs in metal systems, e.g., Ni-doped W; and surficial amorphous films (SAFs). Despite the partial structural order within them, these intergranular or surficial films are often referred to as "glassy" or "amorphous."

Systematic data have been collected for several SAF systems, namely, $\mathrm{Bi}_{2} \mathrm{O}_{3}$ on $\mathrm{ZnO}$ (Luo, Chiang, and Cannon, 2005; Luo and Chiang, 2008), $\mathrm{VO}_{x}$ on $\mathrm{TiO}_{2}$ (Qian and Luo, 2007) and $\mathrm{SiO}_{x}$ on Si (Tang et al., 2008), where film stability and thickness have been measured as functions of temperature and composition (or dopant activities). Thus, they can be considered as "instructive systems" to illustrate the thermodynamic stability of the 


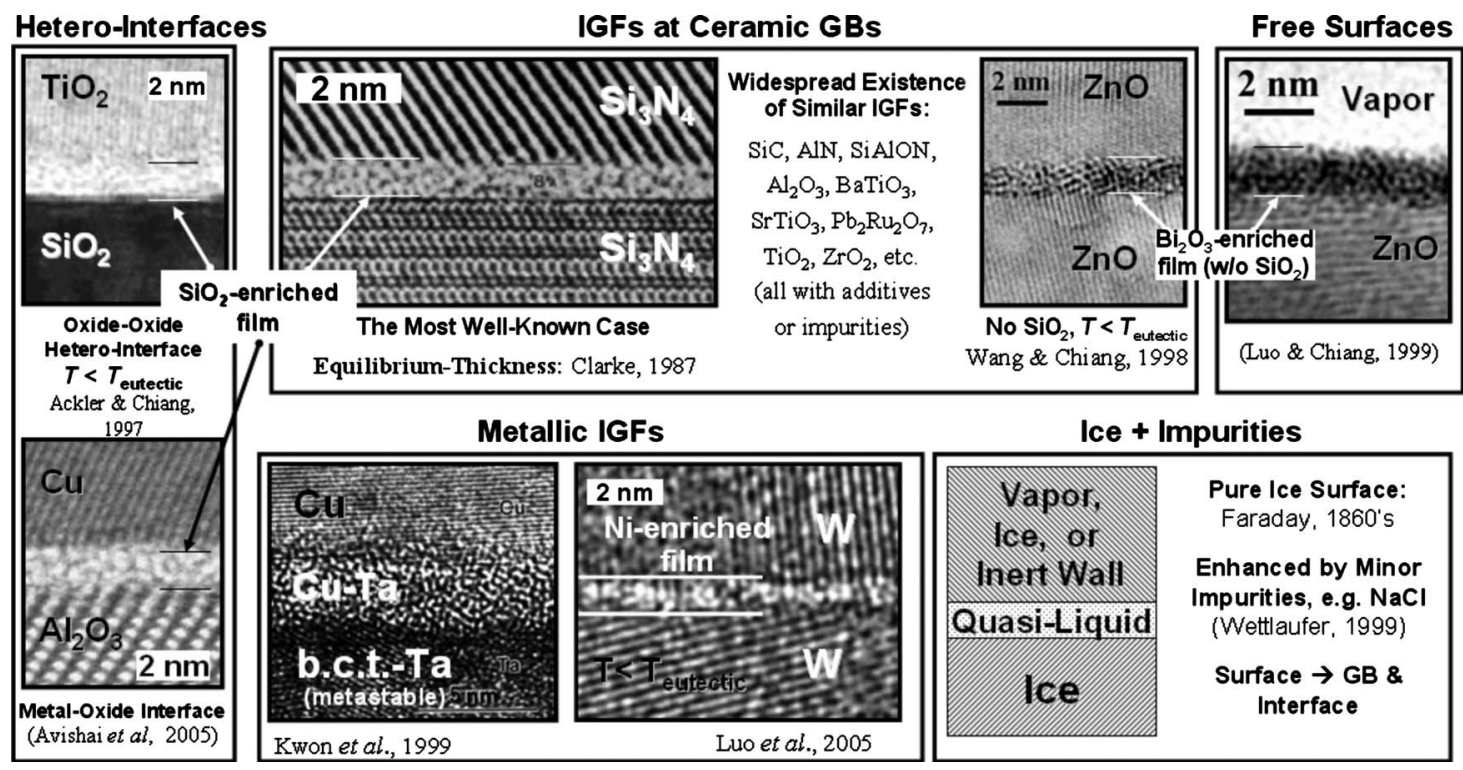

FIG. 13. Representative impurity-based quasiliquid interfacial films. From Luo, 2007.

aforementioned broader class of interfacial films (Luo, 2007).

Experimental observations and thermodynamic models for SAFs in two analogous binary oxide systems $\left(\mathrm{Bi}_{2} \mathrm{O}_{3}\right.$ on $\mathrm{ZnO}$ and $\mathrm{VO}_{x}$ on $\left.\mathrm{TiO}_{2}\right)$ have recently been reviewed (Luo and Chiang, 2008) and are discussed here as illustrative examples. In both systems, the equilibrium film thickness was found to decrease monotonically with decreasing temperature in the subeutectic regimes. Furthermore, dewetting transitions (from a nanoscale SAF to Langmuir submonolayer adsorption) were observed at lower temperatures in both systems. A hysteresis loop in film thickness versus temperature curve was also observed for $\mathrm{VO}_{x}$ on $\mathrm{TiO}_{2}$. This indicates the existence of a first-order monolayer-to-multilayer adsorption transition. Premeltinglike force-balance models (with the volumetric free energy penalty for forming undercooled liquids being the dominating attractive force) predicted subeutectic SAF stability and thickness that agree with experiments for both systems (Luo et al., 2006; Qian and Luo, 2007). This suggests an analogy between the stabilization of subeutectic quasiliquid SAFs in these binary systems and premelting in unary systems (the latter is discussed in Sec. III.C.1). For $\mathrm{Bi}_{2} \mathrm{O}_{3}$ on $\mathrm{ZnO}$, SAFs of similar character were also observed in single-phase $\mathrm{ZnO}$ samples containing $\mathrm{Bi}_{2} \mathrm{O}_{3}$ concentrations below the bulk solid-solubility limits, where the films are thinner.

For $\mathrm{Bi}_{2} \mathrm{O}_{3}$ on $\mathrm{ZnO}$, nanometer-thick SAFs persist into the solid-liquid coexistence regime, in equilibrium with partial-wetting drops (Luo, Chiang, and Cannon, 2005; Luo and Chiang, 2008; Qian et al., 2008), where an analogy to the phenomena of frustrated-complete wetting (Bertrand et al., 2000) and pseudopartial wetting (Brochard and de Gennes, 1991) can be made. The average SAF composition is markedly different from the associated bulk liquid phase even when these quasiliquid SAFs are in thermodynamic equilibration with the bulk liquid phase (Luo and Chiang, 2008).
In a diffuse-interface theory (Luo et al., 2006), these SAFs are alternatively considered as multilayer adsorbates formed from coupled prewetting and premelting transitions. For $\mathrm{Bi}_{2} \mathrm{O}_{3}$ on $\mathrm{ZnO}\{11 \overline{2} 0\}$ surfaces where nanometer-thick SAFs are present in equilibrium with partially wetting drops, the measured contact angle decreases with increasing temperature in the solid-liquid coexistence regime (Qian, Luo, and Chiang, 2008). In contrast, with increasing temperatures the contact angle is virtually a constant on the $\{1 \overline{1} 00\}$ surfaces where SAFs are not present. This observation suggests that wetting in the presence of nanoscale SAFs follows a generalized Cahn wetting model (Cahn, 1977). However, an expected complete wetting transition is inhibited by the presence of an attractive vdW-Ld force of significant strength (Qian, Luo, and Chiang, 2008).

The technological importance of the nanoscale intergranular and surficial films in ceramics and metals is discussed in Sec. IV.A.2.

\section{Charged defects in solids}

As a second example related to solid surfaces and interfaces, space charges related to interfacial segregation of charged defects are discussed here. This is an issue of practical importance for many technological ceramics, where the space charges can often coexist and interact with IGF or SAF formation. Analogous space charges effects are present at both internal interfaces (such as grain boundaries) and free surfaces.

While their individual energies can be calculated by DFT and the LDA, charged defects present several technical challenges because of their long range electrostatic interactions. One in particular is worth describing here. It is frequently observed by electron microscopy that interfaces in ceramics are charged, and these charges are compensated by space charges, in the form of screening distributions of electrons or other mobile 
charged species. Such space charges are expected to have a profound effect on the electronic conductivity and capacitance of boundaries, but their occurrence and extent is unpredictable. The difficulty in understanding and therefore predicting the occurrence of such space charges lies in the length scales involved. The segregation of charged species to the boundary can be understood only at the atomic level. It depends on the details of the atomic structure of the boundary, and atomicscale calculations are necessary to predict the segregation energy. On the other hand, space charges may extend over a length scale up to microns. This is far too large for an atomistic description, and the appropriate physics in this regime is described by a continuum approach, embodied in the Poisson-Boltzmann equation. An example of setting up and solving the PoissonBolzmann equation was given in a study of the energy of a system of mobile charges within an IGF (Johnston and Finnis, 2002).

Consider a planar boundary in the $y-z$ plane. We suppose it somehow lowers its energy by picking up a charge $\sigma$ per unit area. By how much is the energy lowered? We can imagine this as a two-stage process. In the absence of space charge, and omitting electrostatic selfenergy of the segregated charge (which would be infinite in the absence of compensation) it would cost a chemical energy of segregation $E_{\text {seg }}$ to move the charge carriers to the boundary, which must be a negative energy. Then a space charge, consisting in general of positive and/or negative mobile carriers of density $n^{+}$and $n^{-}$, develops to compensate the boundary charge. These carriers have charges $q^{+}$and $q^{-}$. The distribution of the carriers as a function of $x$ in the continuum approximation can be obtained by solving the Poisson-Boltzmann equation. Within the simplest classical density functional theory, assuming the carrier densities are low and linearizing the Poisson-Boltzmann equation, the boundary energy would be lowered by

$$
\begin{aligned}
\Delta \gamma(\text { equil })= & E_{\text {seg }}+\frac{1}{2} V_{H}(0) \sigma \\
& -\frac{1}{2} \int_{0}^{\infty}\left[n^{+}(x) q^{+}+n^{-}(x) q^{-}\right] V_{H}(x) d x .
\end{aligned}
$$

This simple example illustrates how the chemical segregation term is modified by the entropy and separation energy of charged defects. The approach is readily generalized to include the classical correlation energy of the carriers. However, a computational strategy for welding the continuum Poisson-Boltzmann equation onto the discrete atomic sites at a boundary that are available for segregation has yet to be established.

\section{Solid/liquid interfaces and suspensions}

\section{Water and ice}

Water and ice nicely illustrate the effects of LRIs. One phenomenon of water and ice, with significant geophysical and ecological implications, is premelting or surface melting, which can occur at ice surfaces, grain boundaries, and interfaces with inert walls. Furthermore, ice and water can also be used as an instructive system to understand analogous, but usually more complex, interfacial behaviors in multicomponent ceramics and metals, e.g., the impurity-based quasiliquid surficial and interfacial films discussed in Secs. III.B.1 and IV.A.2. Premelting dynamics and its implications are further discussed in Sec. IV.A.3.

Lifshitz theory has had remarkable success in predicting the nature of the surface melting or premelting of many materials (Dash et al., 2006). Of special interest here is ice because of the novel influence of the retardation effects responsible for the change from complete to incomplete surface melting. This is because retardation effects attenuate the LRIs that are driving the film growth (Elbaum and Schick, 1991; Wilen et al., 1995). The basic idea is this. When the polarizability of the substrate is greater than that of the film, wetting occurs. Therefore, when dispersion forces dominate, the wetting of the ice by water at temperatures below $T_{m}$ will be driven when the polarizability of the water lies between that of the ice and the other material (vapor phase or chemically inert solid). However, the net wetting forces depend on the entire frequency spectrum that underlies the polarizability of the system. The novelty of the icewater system, first pointed out by Stranski (1942), is that the polarizability of ice is greater than that of water at frequencies higher than the ultraviolet whereas it is smaller at lower frequencies. Therefore, while the surface melted layer of water is thin, the polarizabilities at all frequencies contribute to drive surface melting. However, when the film thickens, the finite speed of light attenuates the wetting forces by favoring those in which the polarizability of water dominates over that of the ice. Hence, the self-attraction of the water begins to dominate and the film of water on ice stops growing.

We also note here the more familiar mechanisms associated with the extension of the equilibrium domain of the liquid phase into the solid region of the bulk phase diagram: Gibbs-Thomson and colligative effects. Cumulatively we refer to these effects as premelting. Premelting phenomena characterize the equilibrium structure of the material. As noted above, the premelting phenomena observed in ice and other materials have recently been reviewed (Dash et al., 2006), so here we describe only the basic tenets of this aqueous system as they may apply to materials of interest across a range of disciplines.

\section{a. The phase architecture of ice}

Naturally occurring polycrystalline ice holds much in common with all polycrystalline materials but with some distinct advantages for study. It is transparent, optically birefringent, can be easily doped, and can be held near its local melting point without sophisticated cryogenic systems. Like all materials held sufficiently close to their bulk melting points, ice is not entirely solid. Ice has a phase geometry that is characterized by a closely packed hexagonal crystal structure, interlaced with liquid films 


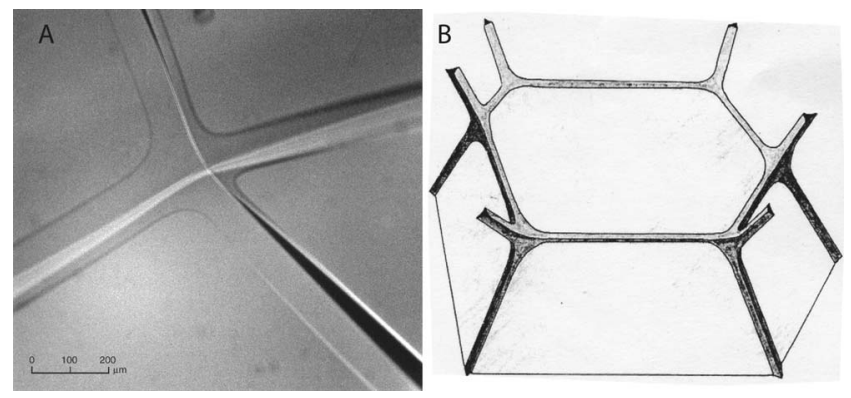

FIG. 14. Liquid film vein structure of polycrystalline ice. Left: Photograph of four veins intersecting at a node between four grains in polycrystalline ice near the bulk melting temperature. Right: Schematic of the vein-node network. From Dash et al., 2006.

threading through its volume. Where three ice grains come together a thin liquid vein exists, and where four grains join a node of liquid water forms (Fig. 14).

The mechanisms responsible for this liquid water are the Gibbs-Thomson and colligative effects. While seen on the scale accessible with an optical microscope there is a finite dihedral angle, but a probe that can penetrate into the grain boundary on a finer scale can assess the conditions under which a film between two grain boundaries, grain boundary premelting, may exist. Namely, moving away from the veins and nodes into the planar interface between two grains, the interfacial curvature effects disappear and the existence of liquid, if present, depends on long ranged intermolecular and electrostatic forces. Here specific models of electrostatic interactions, modeled, for example, using Poisson-Boltzmann theory, can compete admirably with $\mathrm{vdW}$ attraction; they are both required for a complete study of grain boundary melting (Benatov and Wettlaufer, 2004). It was shown there that when the film contains an electrolyte, the solid grains may be held apart by repulsive screened Coulomb interactions against the attractive dispersion interactions, but the latter must be taken out to their full range using Lifshitz theory to ensure that the longest range behavior is captured.

In a thought experiment Benatov and Wettlaufer doped the grain boundary with a 1-1 electrolyte, $\mathrm{NaCl}$. Because missing bonds at any ice surface give rise to an increase in the Bjerrum defect density, they treated the surface as having a finite charge density screened in a manner that depends on the number density of impurity ions. Hence, the detailed consequences rely on a correct treatment of the frequency-dependent dispersion forces and the peculiar functional dependence of the range and amplitude of the repulsive Coulomb interaction on the dopant concentration. In such circumstances, researchers in colloid and interface science usually treat the Debye length as a constant; in these systems this is well justified and it is experimentally realizable. In grain boundary premelting (and indeed surface melting) it is not necessarily realizable. Due to the nearly perfect rejection of salt from the ice lattice the electrolyte remains in the film and thus an increase (decrease) in film thick- ness is accommodated by melting (freezing) of the solid so that, up to the solubility limit, the dopant level is simply inversely proportional to the film volume. Hence, when the film thins at low temperatures the impurity concentration in the film increases and the Debye length decreases through the impurity effect. The novelty then is that the Debye length, and the amplitude and range of the Coulomb interaction, are themselves a function of temperature through the dilution or concentration of the film. This influences the abruptness of the transition from premelted to dry grain boundaries. In the surface melting of ice this same confluence of effects is at work (Wettlaufer, 1999) and is the most likely explanation of the wide variation of experimental data across laboratories that use the same methodology (Dash et al., 2006).

\section{b. Optical properties of ice and water}

As described, the finite travel time of photons is manifested in the incomplete surface melting of ice against a pure vapor phase. The origin is in the frequency dependence of the polarizabilities of water and ice. Two enormously important tasks lie ahead. First, we need to develop novel and quantitatively accurate methodologies to refine and expand our experimental understanding of these data. At present, we must use limited spectra from a variety of sources to fit the data to a damped-oscillator model (Dash et al., 2006). While it is possible to use ultrahigh vacuum (UHV) to obtain the full VUV optical properties for solid materials, we need modern methods that can obtain such properties for high vapor pressure materials, such as most liquids. This will be impeded by the possibility that the spectra themselves differ in an interfacial environment in which they are ultimately of interest, whereas they may be more easily obtained in bulk samples. It is essential to overcome such impediments if we are to understand the phase behavior not only of ice and water but of mixtures across all colloidal and engineering materials.

\section{Hydration}

The ability to predict the properties of solutes in liquid water, a prerequisite for rationally guiding nanoscale processes in aqueous media, continues to be a challenge. Since the earliest realistic simulation of liquid water (Rahman and Stillinger, 1971), computer simulations have proven to be vital complements to experiments in understanding the phenomenon of hydration. For simulation studies of hydration, the most common target is the excess free energy of hydration $\mu^{\text {ex }}$. The excess free energy is the reversible work done to transfer a solute from some phase (typically the vapor) into liquid water. This quantity directly informs us about the solubility of the solute and, with realistically applied extensions, about the interaction of the solute with other solutes and interfaces.

Though the original perturbative ideas were developed for weak coupling (beyond the reference state), this technique can be used to study hydration of solutes that interact strongly with water. For example, the dif- 
ference in hydration free energy of $\mathrm{Na}^{+}(\mathrm{aq})$ and $\mathrm{K}^{+}(\mathrm{aq})$ is of much interest in the study of ion channels (Asthagiri et al., 2006). In perturbative or coupling-parameter approaches, this free energy change is calculated by introducing many artificial states $X_{i}$ intermediate in properties between $\mathrm{Na}^{+}(\mathrm{aq})$ and $\mathrm{K}^{+}(\mathrm{aq})(\mathrm{aq})$ :

$$
\mathrm{Na}^{+} \rightarrow X_{1} \rightarrow \cdots \rightarrow X_{i} \rightarrow \cdots \rightarrow \mathrm{K}^{+} .
$$

Thus $X_{1}(\mathrm{aq})$ is only slightly different from $\mathrm{Na}^{+}(\mathrm{aq})$, such that $\mathrm{Na}^{+}(\mathrm{aq})$ is a useful reference system. Then the free energy changes between $X_{i}$ and $X_{i \pm 1}$ are estimated and the net change between $\mathrm{Na}^{+}$and $\mathrm{K}^{+}$assessed. (Note that in the limit of a continuum of $X$ we recover the coupling parameter approach.) Such techniques have greatly advanced; these developments are summarized by Chipot and Pohorille (2007).

Though the above techniques are by now standard in computer simulation studies of hydration, in an era of increasing sophistication of simulations including $a b$ initio approaches (cf. Sec. II.A.5), the above approaches are neither physically revealing nor readily applicable. For example, if intermolecular potentials from an $a b$ initio calculation are desired, then no real chemical object corresponds to $X_{i}$. Quantum chemistry can provide information only about how $\mathrm{Na}^{+}$and $\mathrm{K}^{+}$interact with water.

In the past decade or so, a new theoretical approach has been advanced based on the insight that the distribution of potential a solute feels in a solvent can be parsed on the basis of local chemical structures [see, e.g., Chipot and Pohorille (2007)]. These quasichemical generalizations of the potential distribution provide a theoretical framework within which to investigate such problems. These techniques have revealed interesting insights about hydrophobic and hydrophilic hydration.

Several features of the above development are noteworthy. First, $\mu^{\mathrm{ex}}$ is transparently linked to the hydration structures that the solute forms. In fact, the theory allows prediction of the most optimal hydration structures. In the cases of hydration of ions with high charge density, these predictions have been supported by results based on ab initio molecular dynamics simulations (Asthagiri et al., 2005). Second, we avoid all considerations of intermediate states that are not physically realizable. Last, it is possible to study directly configurations resulting from an $a b$ initio simulation, i.e., avoiding the mixing of $a b$ initio and classical types of treatment above. So far for reasons of computational cost this has been done only for a classical model of liquid water (Paliwal et al., 2006). The hydration structures predicted by the primitive quasichemical approach are in good accord with hydration structures observed in $a b$ initio simulations.

A clear understanding of the hydration of various ions and their role in biological structures and long range interactions in biological self-assembly is still elusive, despite the fact that over a century has transpired since the first identification of these effects (Kunz et al., 2004). Whereas ab initio simulations of ions in water can provide chemical insights, they are still much too expensive and approximate for large-scale simulations. An interesting development in the area of simulations has been the use of classical potentials that attempt to include polarization [see, e.g., Grossfield et al. (2003)]. Such polarization effects have been shown to be important in how ions partition near an air-water interface (Jungwirth and Tobias, 2006). These simulation efforts can be complemented by the theoretical directions laid out above and these can begin to better illuminate specific ion effects and thus also hydration in nanoscale science.

\section{Structure and dynamics at oxide/electrolyte interfaces}

The interface between oxides and aqueous solutions controls ionic and molecular adsorption (and thus contaminant transport), mineral dissolution or precipitation kinetics, corrosion rates, heterogeneous catalysis, nutrient and energy supply to bacterial communities, chargetransfer processes, and in deep subsurface settings, fracture propagation and hydrous melt formation. Crystalline phases with oxygen as the dominant anion (oxides, silicates, carbonates, phosphates, etc.) are ubiquitous in natural and industrial environments (Brown et $a l ., 1999)$. In the Earth's crust and in many industrial settings such as nuclear and fossil power plants and in chemical and materials industries, interactions between liquid aqueous electrolytes and oxide surfaces, over wide ranges of temperature, pressure, and chemical and/or mineralogical composition, are the dominant processes controlling mass transport, solution chemistry, and mineral transformations. In many natural and industrial systems, nothing interesting happens until aqueous solutions encounter solid surfaces, and the rates of fluidfluxed reactions are so much greater than anhydrous processes that they completely dominate such subjects as geochemistry and corrosion science.

There is no more fundamental process at oxide/water interfaces than the charging of the surface and the structuring of the adjacent fluid phase due to the undercoordination of atoms at the crystal termination. Typically, when oxide surfaces come into contact with water, monovalent cations are rapidly leached out and multivalent cations immediately react with water to produce a surface that is completely covered with variably protonated oxygens bonded to underlying metal ions of the bulk crystal. Commonly this process is modeled using hypothetical reactions such as the "two-pK" model (Stumm, 1992),

$$
\begin{aligned}
& >\mathrm{SO}^{-}+\mathrm{H}^{+} \Leftrightarrow \mathrm{SiOH}^{0}, \\
& \mathrm{~K}_{\mathrm{H} 1}=\left\{>\mathrm{SiOH}^{0}\right\} /\left(\left\{\mathrm{H}^{+}\right\}\left\{>\mathrm{SiO}^{-}\right\}\right], \\
& >\mathrm{SiOH}^{0}+\mathrm{H}^{+} \Leftrightarrow \mathrm{SiOH}_{2}^{+}, \\
& \mathrm{K}_{\mathrm{H} 2}=\left\{>\mathrm{SiOH}_{2}^{+}\right\} /\left(\left\{\mathrm{H}^{+}\right\}\left\{>\mathrm{SiOH}^{0}\right\}\right],
\end{aligned}
$$

where $>\mathrm{S}$ is a generic surface site, [i] are site fractions, and $\left\{\mathrm{H}^{+}\right\}$is the bulk solution $\mathrm{H}^{+}$activity adjusted for the work of bringing the ion to the charged surface. Here the critical role of water dissociation to supply $\mathrm{H}^{+}$is 
apparent. The solution $p \mathrm{H}$ can often be considered the master variable in aqueous processes and the $p \mathrm{H}$ at which oxide surfaces have equal concentrations of negatively and positively charged surface sites, referred to as the $p \mathrm{H}_{\mathrm{pzc}}$ or point of zero charge (PZC) (Sposito, 1998), is a fundamental parameter, obtainable directly from $p \mathrm{H}$ titrations with careful mass and charge balance. For the generic two-pK surface protonation scheme, $p \mathrm{H}_{\mathrm{pzc}}$ $\equiv \mathrm{PZC}=\frac{1}{2}\left(\log _{10} \mathrm{~K}_{\mathrm{H} 1}+\log _{10} \mathrm{~K}_{\mathrm{H} 2}\right)$, which perhaps explains its popularity. Another fundamental variable is the zeta potential (ZP) measured by electrokinetic methods such as electrophoresis or streaming potential. The $p \mathrm{H}$ at which $\mathrm{ZP}=0$ is referred to as the isoelectric point $\left(p \mathrm{H}_{\text {iep }}\right.$ or simply IEP). For ("indifferent") electrolytes whose cations and anions interact nearly equally with the surface, IEP $\approx \mathrm{PZC}$. The exact meaning of $\mathrm{ZP}$ is, however, more ambiguous and highly model dependent (Hunter, 1989; Knecht et al., 2008). In recent years, new experimental approaches have been developed to determine zeta potential, surface charge density, and PZC's for a number of oxides at temperatures above $100{ }^{\circ} \mathrm{C}$ (Wesolowski et al., 2000; Machesky et al., 2001; Fedkin et al., 2003; Zhou et al., 2003).

The surface charge density at $p \mathrm{H}$ 's other than the PZC is governed by the site densities of the surface species and the screening of surface charge buildup by water dipoles and charged, polar, and/or polarizable species in the solution. Electrostatic screening effects are taken into consideration by any of a number of parallel-plate capacitor-type models of the electrostatic double layer (EDL) (Holm et al., 2001; Poon and Andelman, 2006). However, very few real oxides (with the notable exception of quartz and other crystalline and amorphous forms of silica) exhibit surfaces characterized by the simple stoichiometry of reactions (22) and (23). Rather most oxide and silicate surfaces are characterized by oxygens bonded to as many as three or four underlying metal cations, and these can be dissimilar cations with formal valencies ranging from one to five or more (Koretsky et al., 1998). Furthermore, the activity coefficients of surface sites are typically assumed to be unity and equated to their volume or mass concentration or mole fraction, ignoring steric and electrostatic attractive or repulsive forces that might alter their thermodynamic concentrations (Sverjensky, 2003).

A major advance in predicting the surface charging process has been the development of the multisitecomplexation model (Hiemstra et al., 1996), which applies the Pauling bond-valence principle to calculate the unsatisfied valence of oxygen atoms in specific bonding configurations on oxide surfaces, and incorporates hydrogen bonding with sorbed water molecules to provide a truly predictive capability for estimating surface site densities, PZC's, and the protonation states of individual surface sites. The protonation constant for an individual surface oxygen in this approach depends on its local bonding environment and is defined as

$$
\log _{10} \mathrm{~K}_{\mathrm{H}}=-A\left[V+\Sigma_{\mathrm{S}_{\mathrm{MeO}}}+m\left(s_{\mathrm{H}}\right)+n\left(1-s_{\mathrm{H}}\right)\right],
$$

where $V$ is the formal valence of oxygen $(-2)$, the summation totals the bond-valence contribution to the oxygen from all metal ions of the substrate bonded to the surface oxygen (a function of bond length and charge of the cation), $m$ is the number of donating $\mathrm{H}$ bonds from adsorbed water molecules, $n$ is the number of $\mathrm{H}$ bonds contributed by any hydrogen directly bonded to the surface oxygen to adsorbed water molecules, and $s_{\mathrm{H}}$ is the assumed bond-valence contribution of $\mathrm{H}^{+}$. The $A$ parameter in Eq. (24) is a regression constant derived from a large number of hydrolysis reactions of hydrated metal ions in aqueous solution. Machesky et al. (2001) extended this model to $300{ }^{\circ} \mathrm{C}$ and demonstrated its validity for the very few oxides (magnetite, rutile, zirconia, and nickel ferrite) for which PZC data are available at temperatures above $100{ }^{\circ} \mathrm{C}$. This and other surface protonation models and temperature extrapolation approaches are reviewed by Lützenkirchen (2006), who also provided a review of current "surfacecomplexation-models" describing ion adsorption and descriptions of the ion, charge density, and electrical potential distributions in the EDL based on these simplified concepts. These generally involve GouyChapman approximations for the effect of surface charge density on ion distributions in the diffuse layer, together with various Stern or Helmholtz planes of specific ion binding. Sverjensky and co-workers (Criscenti and Sverjensky, 1999; Sverjensky, 2006; Fukushi and Sverjensky, 2007) analyzed a large database of ion adsorption studies on a large number of oxide and silicate surfaces, mainly conducted at room temperature, using the two-pK surface protonation model and the "triple layer" description of the EDL, providing a semiempirical predictive capability for modeling electrolyte oxide interactions based on Born solvation principles and taking into consideration the dielectric properties of the substrate as well as the solution.

All such electrostatic and structural models of the EDL are characterized by numerous adjustable parameters (capacitance terms, specific ion binding constants, estimations of the solvent dielectric properties in the double layer, etc.). They generally lack a predictive capability for Stern layer capacitances and ion binding energies, and the defining parameters are highly covariant and difficult to render physically meaningful, even at room temperature where abundant experimental data are available. Most importantly, they are largely based on hypothetical interfacial structures that ignore the discrete atomic nature of the interface at the angstromnanometer scale. In recent years, there has been a concerted effort to elucidate the actual structure and dynamics of the oxide/water interface using a variety of analytic and computational approaches. Surface forces have been directly measured to determine the dynamics of the electrolyte layer between mica sheets brought into nanometer-scale contact (Zhu and Granick, 2001; Raviv and Klein, 2002). Synchrotron-based extended $\mathrm{x}$-ray-absorption fine structure, $\mathrm{x}$-ray standing wave, and 


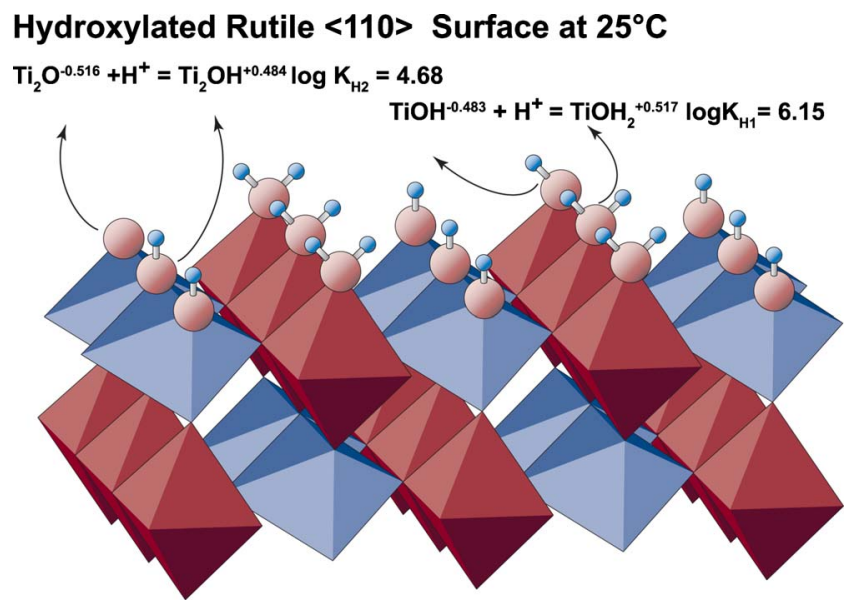

FIG. 15. (Color online) Protonation constants of bridging $\left(\mathrm{Ti}_{2} \mathrm{O}^{-0.516}\right)$ and terminal $\left(\mathrm{TiOH}^{-0.483}\right)$ oxygen atoms on the rutile $\langle 110\rangle$ surface calculated using ab initio-optimized bond lengths and surface atom partial charges as input into Eq. (30). An $\mathrm{O}^{\mathrm{II}}$ ion resides at the corner of each coordination polyhedron, which also contains a central $\mathrm{Ti}^{\mathrm{IV}}$ ion.

reflectivity measurements are being used to map out the three-dimensional (3D) distributions of atoms of the crystal surface, the solvent, and the ionic species in the EDL with sub-angstrom resolution (Fenter et al., 2002; Fenter and Sturchio, 2004). Second harmonic generation (SHG) studies have been applied to determine the point of zero charge of individual crystal faces (Stack et al., 2001; Eisenthal, 2006). To illustrate these approaches, we review recent integrated studies of the interaction of water and aqueous electrolytes with the $\langle 110\rangle$ crystal surface of rutile $\left(\alpha-\mathrm{TiO}_{2}\right)$, perhaps the most intensely studied of all metal-oxide surfaces (Diebold, 2003).

Bandura and Kubicki (2003) used ab initio DFT to calculate the minimum energy configuration of the rutile $\langle 110\rangle$ surface in contact with a significant number of water molecules. Fitts et al. (2005) used the DFT relaxed bond lengths and partial charges of surface oxygen atoms as input into the multisite-complexation model [Eq. (29)] to calculate the protonation constants for the reactive surface oxygen atoms, obtaining a calculated PZC $\left(\sim 5.0\right.$ at $\left.25^{\circ} \mathrm{C}\right)$ in quantitative agreement with SHG measurements of real rutile $\langle 110\rangle$ single-crystal surfaces in contact with dilute aqueous sodium nitrate solutions. Figure 15 shows the protonation scheme for the reactive oxygen atoms on this surface, namely, "bridging" oxygen atoms each bonded to two underlying Ti atoms, and "terminal" oxygen atoms, which result from the chemisorption of water molecules onto bare five-coordinated Ti atoms exposed on the $\langle 110\rangle$ surface. The ab initio optimized surface, and interaction potentials of water and ions with the surface oxygen atoms determined from the DFT calculations were also used by Předota, Bandura, et al. (2004) and Předota and Vlcek (2007) as input into large-scale classical molecular dynamics (MD) simulations of the interface between the rutile $\langle 110\rangle$ surface and $40 \AA$ layers of SPC/E model water (Berendsen et al., $1987)$ at the density $\left(1.0 \mathrm{~g} / \mathrm{cm}^{3}\right)$ of real liquid water.
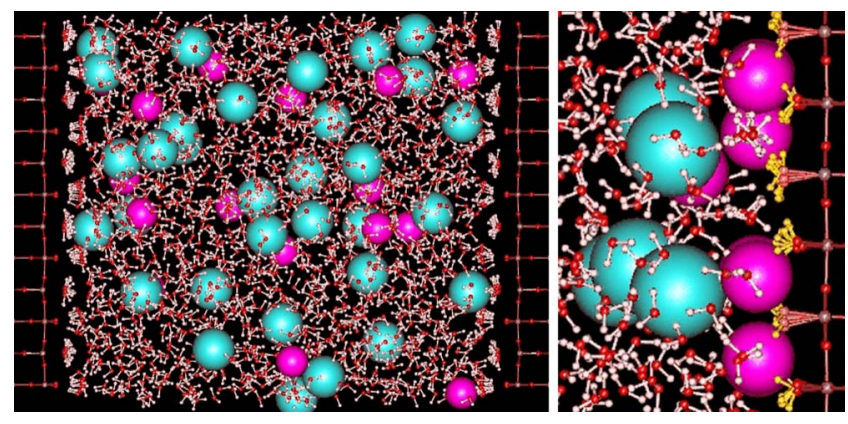

FIG. 16. (Color online) $\sim 1 \mathrm{~m} \mathrm{SrCl}_{2}$ solution (in $\mathrm{SPC} / \mathrm{E}$ water) at $25^{\circ} \mathrm{C}$ at liquid density sandwiched between rutile $\langle 110\rangle$ surface slabs. Oxygen and titanium atoms are minimized for clarity. Also shown are $\mathrm{Sr}^{2+}$ ions, $\mathrm{Cl}^{-}$ions and hydrogen atoms. Left: Uncharged, "nonhydroxylated" surfaces (Předota, Zhang, et al., 2004) with full $40 \AA$ water layer shown. Note strong layering of near-surface water, an inner layer of chemisorbed water molecules atop bare fivefold titanium ions and a distinct second layer associated with the bridging oxygens. Right: Negatively charged $\left(-0.2 \mathrm{C} / \mathrm{m}^{2}\right)$, "hydroxylated" surface attracts $\mathrm{Sr}^{2+}$ ions into "inner sphere" sorption sites, displacing second layer water molecules and interacting directly with bridging and terminal oxygen atoms.

Figure 16 shows typical MD results for SPC/E containing about 2 mol kg-1 dissolved $\mathrm{SrCl}_{2}$ in contact with uncharged and negatively charged surfaces at $1 \mathrm{~atm}$ and 298 K. On the negatively charged surface, the MD simulations predict sorption of solution cations at "inner sphere" sites in direct contact with the surface oxygens. Using synchrotron x-ray standing wave (XSW) and crystal truncation rod (CTR) techniques, Zhang and Glotzer (2004) and Zhang et al. (2007) were able to image the $\langle 110\rangle$ surfaces of real rutile single crystals in contact with real bulk water containing a variety of dissolved ions, at sub-angstrom resolution. As shown in Fig. 17, many ions were found to sorb at a "tetradentate" site in contact with two bridging and two terminal oxygen atoms, while smaller, transition metal cations sorb at "monodentate" and "bidentate" sites that are approximately the same as Ti lattice-equivalent sites in the bulk crystal structure. Figure 18 shows the remarkable agreement obtained from the synchrotron XSW, CTR, and x-ray absorption
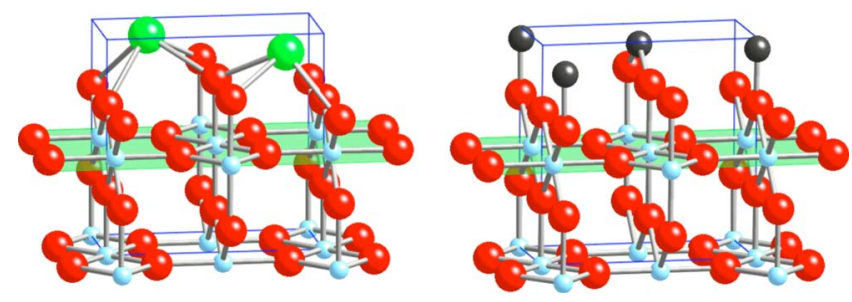

FIG. 17. (Color online) "Inner sphere" sorption site geometries on the rutile $\langle 110\rangle$ surface as identified by synchrotron $\mathrm{x}$-ray standing wave and crystal truncation rod studies. Also shown are oxygen atoms, titanium atoms, and ions sorbed at the "tetradentate" site $\left(\mathrm{Rb}^{+}, \mathrm{Sr}^{2+}, \mathrm{Y}^{3+}\right)$ : "monodentate" and "bidentate" sites preferred by transition-metal cations $\left(\mathrm{Zn}^{2+}, \mathrm{Co}^{2+}\right)$. 


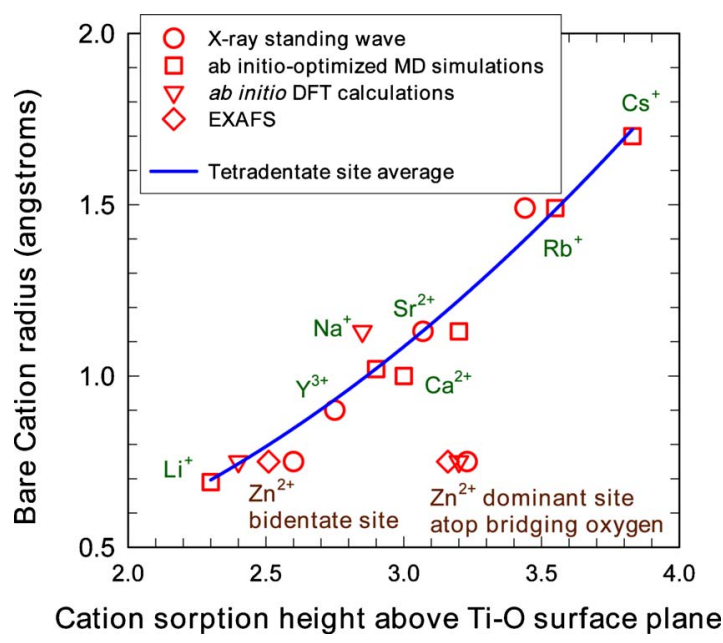

FIG. 18. (Color online) Sorption heights $(\AA)$ of cations above the Ti-O surface plane of rutile $\langle 110\rangle$ vs bare cation radius for ions in the tetradentate, monodentate, and bidentate sites determined by x-ray and computational approaches. From Wesolowski et al., 2008.

fine structure (EXAFS) measurements of real electrolyte solutions in contact with real rutile $\langle 110\rangle$ surfaces, compared with results of the DFT calculations and classical MD simulations. The latter agreement is surprising, given the simplicity of the nondissociating, nonpolarizable SPC/E water model.

These integrated computational, chemical imaging, and macroscopic experimental studies reveal features of the rutile/aqueous electrolyte interface that may be representative of other oxide/water interfaces. The true relaxed state of the crystal surface in contact with bulk water (both real water and SPC/E model water) is shown to be more similar to the undistorted bulk termination than previously indicated from studies of the dry surface under ultrahigh vacuum conditions. The ordering of water molecules adjacent to neutral and charged surfaces is shown to extend only a few monolayers $(\sim 1.5 \mathrm{~nm})$ before bulk solvent properties are observed. The first few water layers are highly ordered, in registry with the bulk and surface crystal structures including strong dipole reorientations and $\mathrm{H}$ bonding within these layers (with no resemblance to liquid water or ice). Cations that sorb as inner sphere complexes in direct contact with surface oxygen atoms are absolutely ordered with respect to the crystal surface structure, and solvent-separated ion pairs further out in the EDL show lateral and axial ordering related to both the crystal structure and the distribution of sorbed water dipoles. These studies provide the first direct evidence that cations of nominally "indifferent" background electrolyte media $\left(\mathrm{Na}^{+}, \mathrm{K}^{+}\right.$, and $\left.\mathrm{Rb}^{+}\right)$also bind at inner sphere sites, competing for sorption at such sites with multivalent trace cations that are much more strongly attracted (much higher binding constants in the thermodynamic sense). These studies also provide fairly direct confirmation of the general features of the multisite-complexation model and the Guoy-ChapmanStern models of surface protonation and binding of

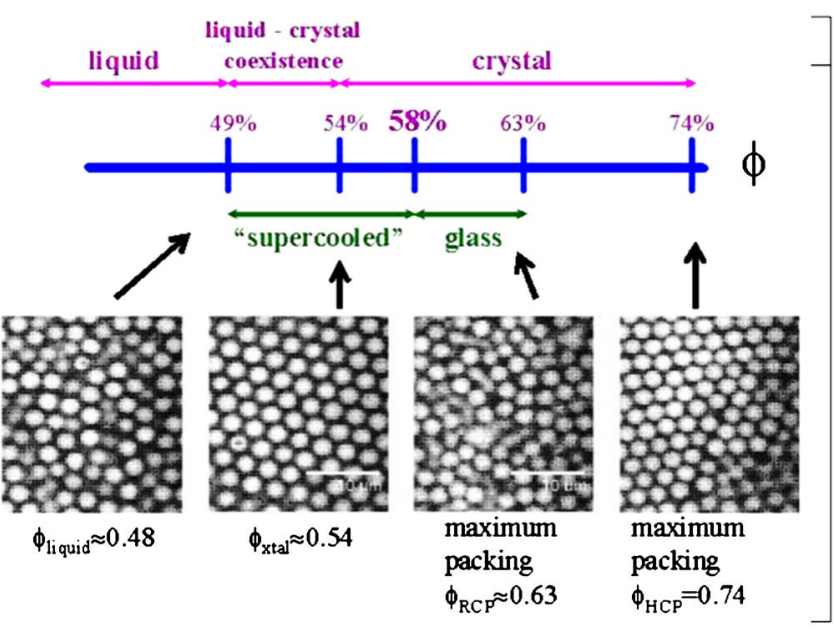

FIG. 19. (Color online) Phase diagram of hard-sphere colloids. Micrographs and schematic plots of $g(r)$ for representative colloidal dense liquid, crystal, and glass suspensions.

counterions in discrete and well-defined layers that screen most of the surface charge within $1 \mathrm{~nm}$ of the surface.

The following needs emerge as necessary at this stage in our learning: more accurate water models; calibration of interactions of ions and surfaces with these model waters for possible use in large-scale molecular dynamics simulations; measurements of ion adsorption and surface charging at high temperatures and pressures as well as in low-density supercritical water; development of parallel codes for making high-level static and dynamic quantum mechanical calculations of interfaces involving meaningful numbers of solid and solution species, that will capture the collective (nonpairwise) interactions of complex systems; and chemical imaging of interfacial structures and dynamics at the sub-angstrom to micron scales.

Similar ideas, for metal/electrolyte interfaces, are beginning to appear in integrated experimental and computational studies of such interfaces [see, e.g., Ogasawara et al. (2002); Denzler et al. (2003); Schiros et al. (2007)].

\section{Colloidal suspensions}

What can we learn from traditional colloidal building blocks? Before increasing system complexity, it is instructive to examine the behavior of model colloidal building blocks composed of either hard or attractive (i.e., sticky) spheres. The phase behavior, structure, and dynamics of colloidal suspensions composed of traditional building blocks depend both on the colloid volume fraction $\phi$ and on their interparticle interactions. In hard-sphere suspensions, the critical parameter for determining phase behavior is $\phi$ (Prasad et al., 2007); see Fig. 19. For $\phi$ just below 0.49, the suspension forms a dense liquid with particle positions that are disordered. The radial distribution function $g(r)$ is a measure of the probability of a particle center being located at a distance $r$ from a given particle, relative to a uniform dis- 

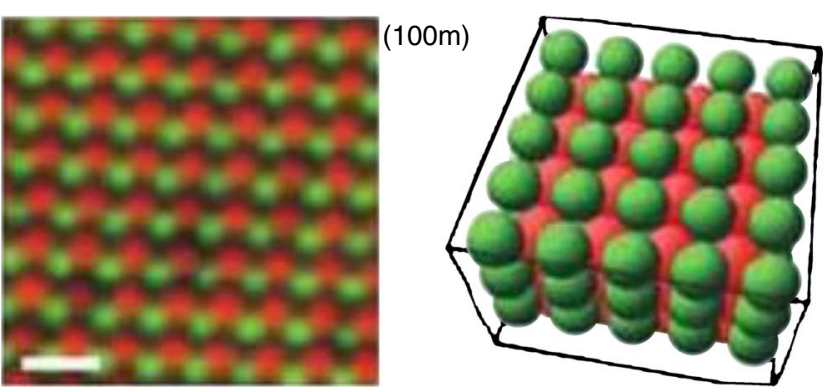

FIG. 20. (Color online) Ionic crystal $(\mathrm{CsCl})$ assembled from oppositely charged colloidal microspheres. From Leunissen et al., 2005.

tribution. For a dense colloidal fluid approaching strong correlation in spacings, $g(r)$ contains local maxima and minima at integral multiples of the interparticle spacing. As $\phi$ is increased toward 0.49 , the particle positions become increasingly correlated, as shown in the micrograph of a colloidal fluid suspension (Fig. 19). When $\phi$ is increased above 0.49 and the system is allowed to equilibrate, entropy drives the formation of crystalline domains. From $\phi=0.49$ to 0.54 , the liquid and crystal phases coexist. Above $\phi=0.54$, the state of the suspension is a crystalline solid with a high degree of positional order, as shown in Fig. 19. The sharp maxima in the crystal $g(r)$ correspond to spacing of particles within this ordered structure. By contrast, when $\phi$ is increased above 0.49 in systems that are not allowed to equilibrate, the suspension resides in a supercooled fluid state, which is a metastable solid that eventually relaxes. From $\phi$ $=0.58$ to 0.63 , the suspension retains its glassy nature and is unable to relax over the experimental time frame. The particle arrangements in colloidal glasses closely resemble those of liquids (Fig. 19). Similarly, $g(r)$ for a glass is like that of a dense fluid, with correlations extending over multiple coordination shells. What distinguishes a glass from a liquid are the particle dynamics; local caging of particles leads to dynamic arrest in the solid glass, whereas in the liquid the particles freely rearrange. The repulsive potential in soft-sphere systems increases the particle's effective size and therefore phase transitions occur at lower $\phi$, while the liquid-crystal coexistence phase is relatively smaller than in hard spheres (Liu et al., 2002).

Colloidal mixtures exhibit rich phase behavior. The simple act of mixing two particle populations together can lead to unexpectedly rich phase behavior. For example, van Blaaderen and co-workers (Leunissen et al., 2005) demonstrated that oppositely charged microspheres can self-assemble into ionic crystals under appropriate solution conditions. Figure 20 shows a $\mathrm{CsCl}$ lattice assembled from $1 \mu \mathrm{m}$ spheres that are weakly positive and negative. In another important example, Lewis and co-workers (Mohraz et al., 2008) recently developed biphasic mixtures of attractive and repulsive microspheres, in which both the structure of colloidal gels formed by the attractive species and the dynamics of the repulsive species can be tuned solely by varying the ratio of the two constituents.

As the above examples illustrate, there is still much to learn even in colloidal systems based on simple mixtures. Nevertheless, there is a strong drive to introduce greater complexity by tailoring colloidal building blocks at the subparticle level. Manipulating colloids and selfassembly via harnessing LRIs are discussed in Sec. IV.B.

\section{Solution-based manipulation of SWCNT}

Carbon nanotubes have been studied intensively over the last 15 years. Their physical and electronic structure is well known as are many other characteristics such as elasticity, strength, transport, and optical properties (Saito et al., 1999). There are several optical techniques including absorbance, Raman, circular dichroism, and fluorescence spectroscopy that have been developed to help study and identify CNTs. Scanning probe techniques such as STM and AFM have been used widely to study their structure and properties.

How can manipulation of LRIs be used in solutionbased processing of single-walled carbon nanotubes (single layers of carbon atoms rolled into a seamless tube with diameter of about $1 \mathrm{~nm}$ )? To first order, if one regards SWCNT band structure as that of graphite with some $k$ vectors disallowed due to circumferential symmetry, one finds that two-thirds of all allowed SWCNT have a band gap and the remaining are metallic (Saito et al., 1999). This fact is simultaneously the bane and the promise of these materials. While each form has highly desirable properties, all known syntheses result in some mixture. The ability either to synthesize a known type, or to sort individual types in a mixture, is therefore a critical problem. For electronic applications, there are two broad approaches. One may either grow the SWCNT on a substrate (Javey et al., 2003) or process it in a liquid and attempt to place it on the substrate (Arnold et al., 2006; McLean et al., 2006). In the latter case, the process is almost invariably governed by long range interactions such as between the SWCNT and a substrate or another interface. Here we focus our attention on solution-based processing which is applicable more generally.

Noncovalent modification using surfactants and biological molecules (Bachilo et al., 2002; Zheng, Jagota, Semke, et al., 2003; Zheng, Jagota, Strano, et al., 2003) works with long range interactions to create solutionbased manipulation techniques. Several solution-based techniques for sorting by metallic versus semiconducting character, or by diameter, have also been demonstrated (Weisman, 2003) based on selective adsorption (Chattopadhyay et al., 2003), dielectrophoresis (Krupke et al., 2003), density differentiation (Arnold et al., 2006), and using a DNA-CNT hybrid (Zheng, Jagota, Semke, et al., 2003; Zheng, Jagota, Strano, et al., 2003; Tu et al., 2009).

We discuss how control of long range interactions in solution has contributed to solving problems associated with dispersion, sorting, and placement of SWCNT. We consider approaches where dispersion is achieved by 
forming a hybrid of the SWCNT with small-molecule or polymeric surfactants and focus on a few successful examples.

\section{a. Dispersion and structure}

Surfactants have been used to disperse SWCNTs in water. These include molecules with charged head groups and flexible alkyl tails such as sodium dodecyl sulfate (SDS) (Bachilo et al., 2002) and, more rigid, planar surfactants such as sodium cholate (SC), sodium deoxycholate, and sodium taurodeoxycholate (Arnold et al., 2006). A signature of successful dispersion is the appearance of distinct band-gap absorbance peaks in the near infrared, and of corresponding band-gap fluorescence. Resulting dispersions are stable as long as sufficient excess surfactant is maintained.

Qualitatively, the nature of the hydrophobic interaction that controls surfactant-SWCNT hybrid formation can be readily understood by analogy with micelle formation. However, there are two basic issues about which there is currently little quantitative understanding: the structure of the resulting hybrid and the binding strength. Knowledge of the structure determines the effective density of the hybrid material, and this is a delicate matter. Small differences in density have been invoked to explain the basic dispersion mechanism, i.e., why individual SWCNT hybrids remain suspended while small clusters are dispatched to the bottom of the centrifuge tube. More importantly, as discussed next, modulation of the effective density of the SWCNT-surfactant hybrid both by the diameter of the SWCNT core and by its electronic properties (e.g., metallic versus semiconducting character) allows their separation by centrifugation in a density gradient. Direct observation of the structure is difficult and has rarely been reported. Experimental and theoretical studies of the structure and binding thermodynamics and kinetics are much needed. The structure seems to depend on the electronic character of the core SWCNT and suggests that electronic response to charges on the surfactant plays an important role.

Dispersion of SWCNT has also been demonstrated by coating with water-soluble polymers (O'Connell et al., 2001; Zheng, Jagota, Semke, et al., 2003). As for smallmolecule surfactants, pertinent issues relate to the binding free energy and the structure of the resulting hybrid. DNA-dispersed SWCNT (DNA-CNT), because the two constituents themselves have been studied extensively, may prove to be a good model system.

Single and double-stranded DNA have been studied even more extensively than CNTs. It is believed that the interaction between the DNA and SWCNTs is mediated by stacking of bases onto the CNT side-wall and that the entire hybrid is rendered water soluble because of the charged sugar phosphate backbone (Fig. 21). By subjecting it to a combination of ion-exchange and size exclusion chromatography, one can obtain excellent model systems, for instance, consisting primarily of one type of CNT with controlled length wrapped by ssDNA with known sequence and length (Zheng, Jagota, Semke, et al., 2003; Zheng, Jagota, Strano, et al., 2003; Tu et al., 2009; see Fig. 21).

Various contributions to the free energy of binding of DNA homopolymers to a SWCNT have been discussed in Manohar et al. (2007). Dispersion efficiency appears to be optimal for sequences with about 30 bases although it has also been achieved with small dsDNA molecules (6-mers) (Vogel et al., 2007). Dispersion by individual nucleotides, which is not as effective as with longer strands, shows that purines are more effective than pyrimidines and that charge on the phosphate plays an important role. Binding strength decreases in the sequence guanine $>$ adenine $>$ (cytosine/thymine/urasil) (Ikeda et al., 2006). Similar findings have been reported for binding of bases onto graphite; the sequence is $G$ $>A>T>C$ (Sowerby, Cohn, et al., 2001; Sowerby, Morth, and Holm, 2001) with binding enthalpy of adenine reported to be about $20 \mathrm{~kJ} / \mathrm{mol}$. Since the nominal Kuhn length of ssDNA [ 1.6 nm (Bustamante et al., 2003)] includes several bases, this suggests that binding is strong enough to overcome entropic increase in free energy, and that all bases should be bound. However, experiments find that the binding strength of homopolymers follows a different sequence $(T>A>C)$ and simulations suggest that the difference is because steric hindrance between adjacent bases results in only partial base stacking with effective binding strength in the sequence $T>G>A>C$. That the DNA binds in helical fashion has been suggested by molecular models (Johnson, Johnson, and Klein, 2008) and confirmed by AFM (Zheng, Jagota, Semke, et al., 2003; Zheng, Jagota, Strano, et al., 2003). It has been suggested that certain structures are further stabilized by unconventional base pairing (Saenger, 1984; Zheng, Jagota, Semke, et al., 2003; Zheng, Jagota, Strano, et al., 2003; Tu et al., 2009). For example, adenine is known to form hydrogenbonded monolayers on graphite (Sowerby, Cohn, et al., 2001). There is currently a lack of measurements on binding free energies and structure.

Single-stranded DNA in water carries a charge on its backbone about every $0.6 \mathrm{~nm}$. On wrapping around the CNT, it therefore renders the hybrid a highly charged rod. Therefore, electrostatics is expected to play an important role in determining the structure, in sorting, and during placement. Handling electrostatic interactions for DNA-CNT, even within the Poisson-Boltzmann approximation, raises new questions because of the high linear charge density and the variable electronic properties of the SWCNT core. This issue will be discussed further in the following subsection.

\section{b. Sorting and placement}

The ability to create hybrids of surfactant and polymeric molecules with individual SWCNT has made it possible to sort dispersions. While techniques based on covalent modification of the SWCNT have been demonstrated, we consider only those that rely on noncovalent (long range) interactions. Separation by metallic versus semiconducting character and by diameter has been 


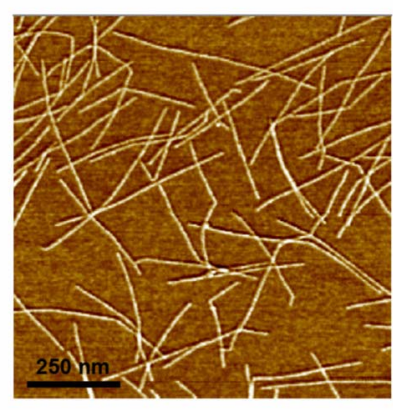

(a)

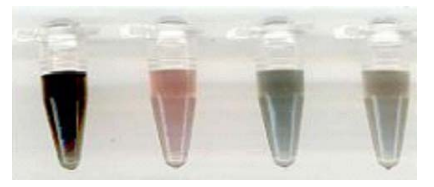

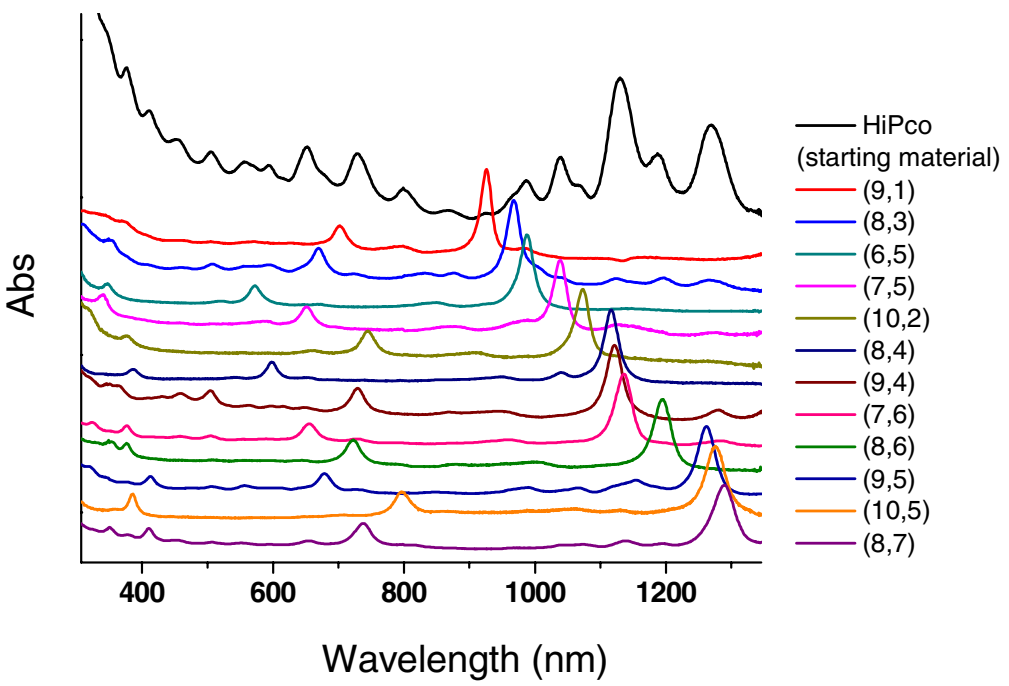

(b)

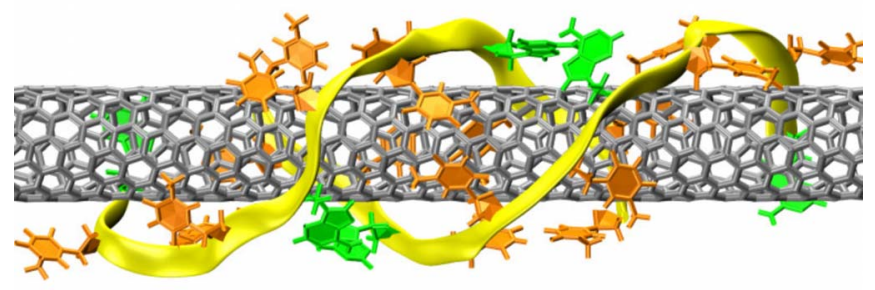

(c)

(d)

FIG. 21. (Color online) DNA forms a hybrid with carbon nanotubes, enabling their dispersion and separation. (a) Atomic force microscopy image of DNA-CNT deposited on a mica surface following separation and length fractionation. (b) Absorption spectra of all 12 semiconducting species separated by ion-exchange chromatography of a starting mixture (top plot) using different DNA sequences. (c) A starting dispersion (black) and three sorted fractions rich in metallic SWCNT and semiconducting SWCNT with different diameters. (d) Proposed $\beta$-barrel structure of the DNA-CNT hybrid. (a) Adapted from Zheng and Semke, 2007. (b) and (d) Adapted from Tu et al., 2009. (c) Adapted from Zheng, Jagota, Semke, et al., 2003.

demonstrated by differential adsorption (Chattopadhyay et al., 2003); by dielectrophoresis (Krupke et al., 2003) that differentiates based on difference in polarizability with respect to that of water, by density differentiation (Arnold et al., 2006); and by ion-exchange chromatography (Zheng, Jagota, Semke, et al., 2003; Zhang, Jagota, Strano, et al., 2003; Tu et al., 2009).

Figure 22 shows that hybrids of sodium cholate with SWCNTs can be separated by ultracentrifugation in a medium with a density gradient (Arnold et al., 2006). SC/SWCNT hybrids travel to the location in the medium that has the same effective density; separation is therefore an indication that hybrid effective density depends systematically on SWCNT diameter and electronic properties. As discussed previously, little is known about how interactions between the SC molecules and SWCNT control structure. For DNA-CNT, ion-exchange chromatography is understood to differentiate between SWCNT cores because the latter modulates the electrostatic interactions of the hybrid with external substrates or fields (Lustig et al., 2005). In both cases, it is likely that hydrophobic, van der Waals, and electrostatic inter- actions will all play an important role. Much more work is needed to understand how the charged SWCNT rod interacts with an external field, with other rods, and with a substrate (either for deposition or for sorting).

The examples cited here work by converting the SWCNT into a hybrid with an amphiphilic molecule (surfactants or polyelectrolytes). Systematic variations in the structure and effective electrostatics of the hybrid rely on delicate control of long range interactions between the constituents of the hybrid, and between the hybrid and external fields or substrates. Both because of the interest in this application and because SWCNT hybrids provide model systems, there is scope for considerable further work on these materials to understand how they work and to improve dispersion, sorting, and placement techniques.

\section{HARNESSING LRIs}

\section{A. Surfaces and interfaces}

The interface between solids and liquids (or quasiliquids) is a complex region where electrostatic, vdW-Ld, 
a

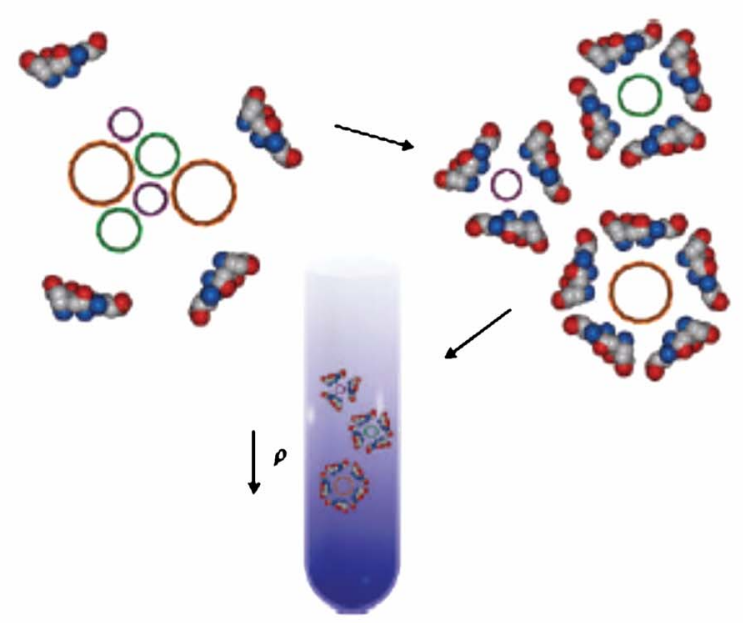

b

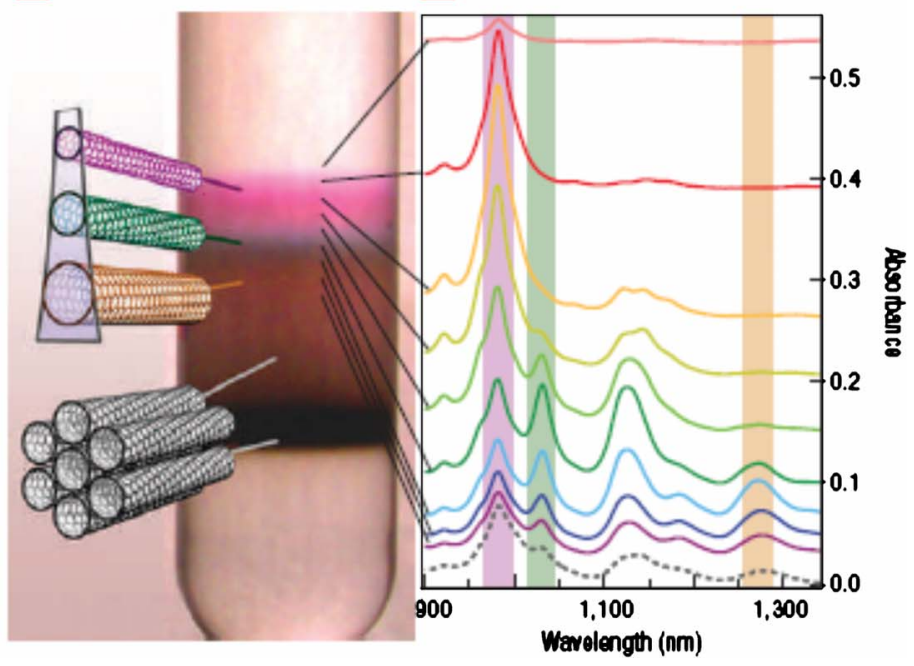

FIG. 22. (Color online) Centrifugation of surfactant (sodium cholate) dispersed SWCNT in a density gradient has been demonstrated to result in their separation. It has been proposed that the effective density of the surfactant-SWCNT hybrid increases with increase in diameter and on the electronic character of the SWCNT. From Arnold et al., 2006.

polar $(A B)$, solvation, steric, and related interactions create distinct properties dictated by the nature of the bounding bulk phases and by temperature and pressure. This region is often poorly characterized at the atomic level. In this section, three examples are given of the potential to manipulate such interfaces for desired properties.

We first discuss proton-conducting polymer membranes, a system whose function is entirely dictated by an interplay of long range forces and interactions. The hope is that these can be manipulated and tailored to optimize performance. However, such "tailoring" has been achieved largely by trial and error. Many variables, such as polymer backbone, side chain and functional group properties and distributions, and the type, size, and distribution of inorganic additives and processing methods are simply altered by many hundreds of researchers in search of ideal characteristics.

Second, we discuss the formation of amorphous intergranular and surface films during ceramic and metals processing. The thickness, composition, and structural or dynamic properties of such films are clearly driven by the bulk compositions and structures of the substrates on which they form, by trace levels of contaminants that often migrate to these films, and by environmental parameters such as temperature, pressure, and their gradients. Intergranular films strongly influence the toughness, strength, permeability, and many other physicochemical properties of the processed material, and surface films can alter catalytic activity, resistance to corrosion, or sequestration of contaminants. As for ionconducting membranes, an atomic-level predictive understanding of the origins and controls on intergranular and surface film structures and properties would enable efficient manipulation of input parameters for desired functionality.
Finally, we describe the more general phenomenon of premelting at grain boundaries and surfaces. Premelting is driven by temperature and compositional gradients, by juxtaposition of particles with different crystallographic orientations or bulk properties, and by the interplay of forces at their interface so as to induce the formation of a thin liquid film at a temperature below the $T_{m}$ of the bulk phase. It is shown that manipulation of temperature and compositional gradients in such systems might be used to redistribute nanoparticles embedded within a polycrystalline material during warming, or from a particle suspension in a liquid during cooling. One intriguing consequence of premelting in polycrystalline ice is the interpretation of paleoclimate from the record of trapped water, trace elements, and gases in continental glaciers. If neglected, the consequences of premelting phenomena can lead to erroneous interpretations of the evolution of the Earth's atmosphere, hydrosphere, and climate spanning geologic time scales.

\section{Proton exchange membranes for hydrogen fuel cells}

In a hydrogen fuel cell, $\mathrm{H}_{2}$ is catalytically split into protons and electrons at an anode (usually Pt nanocatalyst on a carbon support). Protons traverse a protonpermeable membrane, where they are catalytically recombined at a cathode with $\mathrm{O}_{2}$ and electrons to form water. Electrons perform work in an external circuit, for example, to power a hybrid vehicle or a static power generator. Particularly for transportation applications, novel proton exchange membranes (PEMs), to serve as proton conductors as well as electrical insulators and barriers to fuel-oxidant mixing, are needed for fuel cells that operate efficiently and reliably from $-40{ }^{\circ} \mathrm{C}$ to $130{ }^{\circ} \mathrm{C}$ and at low relative humidities (Kreuer et al., 2004; Eikerling et al., 2006). The rational design of advanced membranes requires control and understand- 


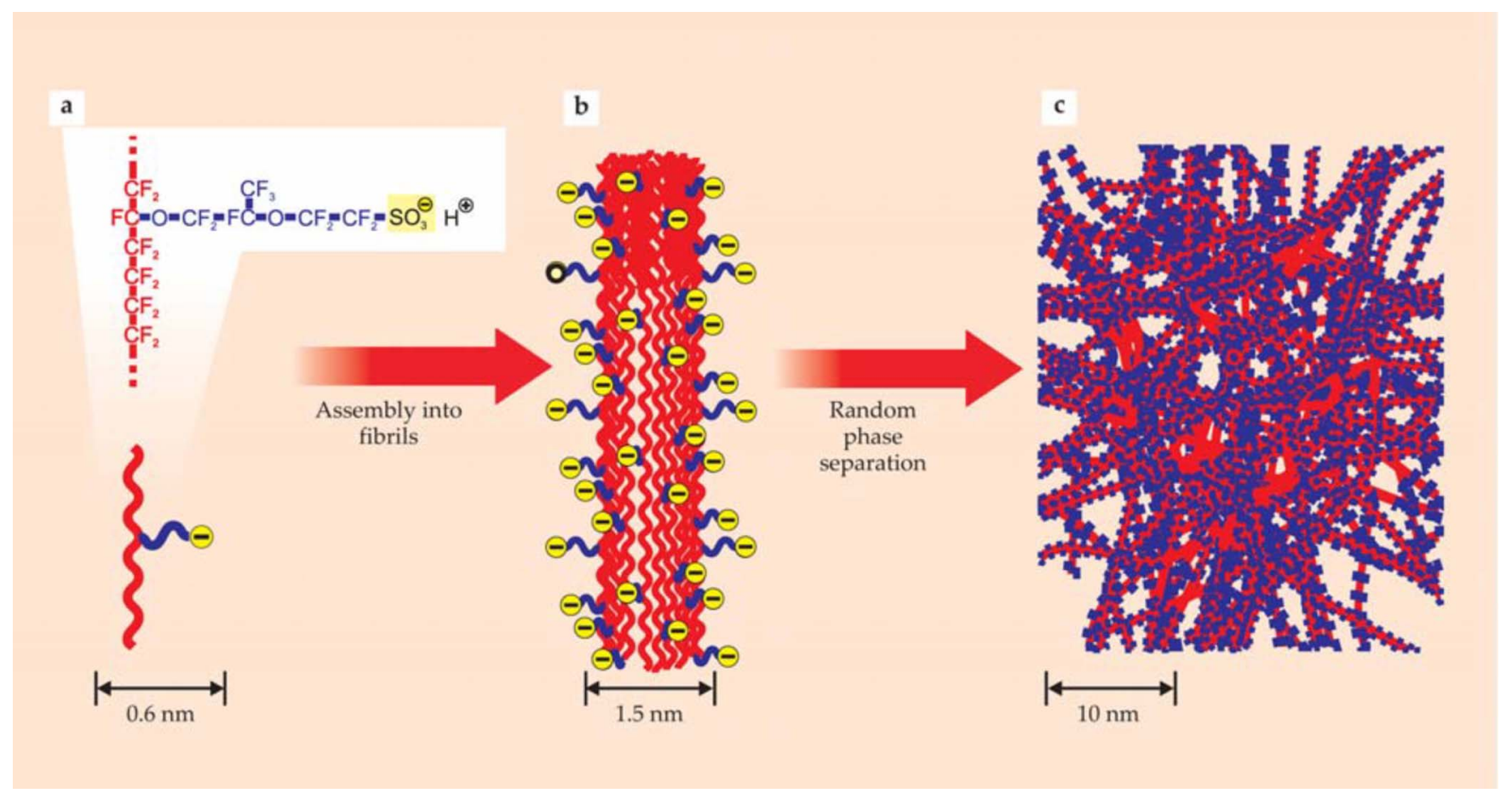

FIG. 23. (Color online) Hypothetical Nafion membrane self-assembly. From Eikerling et al., 2006.

ing of membrane structure and dynamics at the nanoscale. Exploiting unique phenomena resulting from reactive interactions in such a complex, multicomponent, multiscale system is a scientific and technical challenge.

Nafion ${ }^{\circledR}$, the industry standard PEM (Mauritz and Moore, 2004), performs poorly at $>80{ }^{\circ} \mathrm{C}$ and $<100 \%$ relative humidity. Other ionomers are being considered for PEM fuel cells (Hickner et al., 2004; Harrison et al., 2005; Hickner and Pivovar, 2005), but they all fail to meet required performance characteristics. Nafion is a statistical copolymer of tetrafluoroethylene and tetrafluoroethylene substituted with pendant perfluoroether chains terminated with sulfonic acid groups. This material is highly polydisperse, molecular weight is not well known, and placement of the sulfonic acid groups along the backbone is not readily controlled. Although statistical copolymers usually do not form multiphase morphologies, in the case of Nafion the very high incompatibility between the two types of units does result in a nanometer-sized phase-separated morphology. Figure 23 shows the (hypothetical) complexity of Nafion-like random copolymer membranes at atomic to macroscopic scales. Attempts to model structure and dynamics of such membranes at these scales have thus far met with limited success (Jang et al., 2004; Kreuer et al., 2004; Blake et al., 2005).

The morphology assumed by uncharged diblock copolymers reflects, to a good approximation, only the volume fraction of the two components (Khandpur et al., 1995; Bates and Fredrickson, 1999). The excludedvolume and component-specific affinity effects of inorganic nanoparticles on diblock copolymer morphology, chemical and mechanical stability have been clearly demonstrated for the simpler case of nanoparticle- guided self-assembly of uncharged block copolymers (Balazs et al., 2006). Such approaches have not been extensively applied to pure ionomer or inorganic composite membrane systems.

The addition of inorganic particles (oxides and other compounds of $\mathrm{Al}, \mathrm{Mo}, \mathrm{P}, \mathrm{Si}, \mathrm{Ti}, \mathrm{W}, \mathrm{Zr}$, etc.) to Nafion (and to a lesser extent other PEMs) can dramatically improve PEM fuel cell power output, membrane water uptake, and retention and membrane tensile strength while reducing polymer decomposition and fuel, oxygen, and water transport (Alberti and Casciola, 2003; Alberti et al., 2005; Chalkova et al., 2006; Licoccia and Traversa, 2006). Inorganic additives generally increase the glass transition temperature of the polymer (Adjemian et al., 2006), suggesting a correlation with improved hightemperature performance. Surface functionalization of inorganic additives provides the opportunity to tailor the surface to achieve desired characteristics (Gomes et al., 2006; Kim et al., 2006). Since the polymer functional groups are highly acidic, releasing $\mathrm{H}^{+}$to form sites of fixed negative charge, one could reasonably postulate that inorganic phases that become positively or negatively charged at the low- $p \mathrm{H}$ operating conditions might alter PEM performance through electrostatic interactions with the functional groups. Inorganic additives also exhibit a wide range of bulk dielectric constants $(\varepsilon)$, such as 120 for rutile $\left(\alpha-\mathrm{TiO}_{2}\right)$ to 4.6 for quartz $\left(\alpha-\mathrm{SiO}_{2}\right)$ (Sverjensky, 2001). Both electrostatic and vdW-Ld interactions of inorganic nanoparticle and macroparticle additives with both charged and uncharged regions of ionomer membranes may dictate the structure, dynamics, and performance characteristics of composite membranes for fuel cell and similar applications. 


\section{Intergranular and surficial films}

Impurity-based, nanometer-thick intergranular films (IGFs) have been widely observed in ceramics and metals. Clarke originally proposed that these IGFs exhibit an equilibrium thickness (Clarke, 1987). A recent critical review (Luo, 2007) pointed out the following general character of IGFs: First, IGFs exhibit a self-selecting (equilibrium) thickness for a given set of equilibration conditions, and the equilibrium thickness can be altered by changing the temperature, dopant activities, and other thermodynamic potentials. Second, the average composition of an IGF differs markedly from that of the bulk liquid phase, and it can lie within a bulk miscibility gap. Third, quasiliquid IGFs can be stabilized at subsolidus temperatures. Finally, IGFs generally exhibit partial structural order and through-thickness compositional or structural gradients.

Surficial amorphous films (SAFs) of similar character have been observed (Luo and Chiang, 2008). Systematic data have been collected for two SAF systems, providing insights into understanding analogous IGFs where systematic experiments of a similar level have not been conducted. These observations are reviewed in Section III.B.1.

In a phenomenological model, the excess film free energy is written as

$$
\begin{aligned}
G^{x}(h)= & \left(\gamma_{1}+\gamma_{2}\right)+\left(\Delta G_{\mathrm{vol}} h\right) \\
& +\frac{-A}{12 \pi h^{2}}+\sigma_{\text {elec }}(h)+\sigma_{\text {short range }}(h)+\cdots .
\end{aligned}
$$

The term $\gamma_{1}+\gamma_{2}$ represents the sum of the excess free energies of two independent (well separated) interfaces $\left[\left(\gamma_{1}+\gamma_{2}\right)=2 \gamma_{c l}\right.$ for an IGF or $\left(\gamma_{1}+\gamma_{2}\right)=\gamma_{c l}+\gamma_{l v}$ for an $\mathrm{SAF}$. $\Delta G_{\mathrm{vol}}$ is a volumetric free energy for forming a hypothesized uniform liquid film from a mixture of equilibrium bulk phases. When the film is thin, additional interfacial interactions may arise, e.g., a vdW-Ld force, an electrostatic double-layer interaction, and short range interactions of structural and chemical origin. Capillary and applied pressures, if they are present, also affect film thickness. An "equilibrium" thickness corresponds to a balance among these attractive and repulsive interfacial interactions, i.e., a local or global minimum in the excess free energy. This model, initially carried over from the wetting community and proposed for IGFs by Clarke (1987), can be viewed as a high-temperature DLVO theory, in which $\Delta G_{\mathrm{vol}} h$ is an additional significant attractive interaction for quasiliquid films formed under subsolidus conditions (being analogous to the volumetric term in premelting theories). Recently significant progress has been made in quantifying vdW-Ld forces (French, 2000). There is a critical need to quantify other interfacial interactions. Further discussions of interfacial interactions can be found in Luo (2007).

Alternatively, these nanometer-thick IGFs and SAFs can be described as multilayer adsorbates with compositions set by bulk chemical potentials (Cannon and Es- posito, 1999; Cannon et al., 2000). These IGFs and SAFs can be understood as graded multilayer adsorbates formed from coupled premelting and prewetting transitions in diffuse-interface models (Luo et al., 2006; Tang et al., 2006) that were extended from the critical point wetting model (Cahn, 1977).

A challenge is to develop quantitative and realistic models to predict the stability of IGFs. In general, systematic measurements of the film thickness and other characteristics as a function of temperature and chemical potentials are required to validate interfacial thermodynamic models. Despite wide observations of IGFs, such measurements have not been conducted because of difficulties in controlling impurities in ceramics and efficiently preparing TEM specimens.

SAFs do offer a good platform to conduct critical and systematic measurements that are difficult to conduct on IGFs. Moreover, vdW-Ld forces, which are always attractive for IGFs of symmetrical configurations, can be repulsive for SAFs. Stable subsolidus SAFs have been found in systems with repulsive vdW-Ld forces. Experiments of SAF film stability and related wetting transitions in these systems provided insights into understanding the thermodynamic stability of IGFs.

The technological importance of SAFs and IGFs has recently been reviewed (Luo, 2007). IGFs play important roles in fracture toughness, strength, fatigue, creep resistance, and oxidation of $\mathrm{Si}_{3} \mathrm{~N}_{4}$ - and $\mathrm{SiC}$-based structural ceramics, mechanical properties and erosive wear behaviors of $\mathrm{Al}_{2} \mathrm{O}_{3}$, superplasticity of $\mathrm{ZrO}_{2}$, tunable conductivities for ruthenate-based thick-film resistors, nonlinear $I-V$ characteristics for $\underline{\mathrm{ZnO}}-\mathrm{Bi}_{2} \mathrm{O}_{3}$-based varistors, and functions of $(\mathrm{Sr}, \mathrm{Ba}) \mathrm{TiO}_{3}$-based perovskite sensors and actuators. IGFs have also been found in Synroc, AlN substrates, and high- $T_{c}$ superconductors, with attendant detrimental implications on corrosion resistance and thermal or electrical conductivity. Understanding and control of SAFs are also technologically important for tailoring supported oxide catalysts and ultrathin dielectric films as well as for manipulating the shape and growth kinetics of nanoparticles and nanowires.

Furthermore, IGFs and SAFs have important technological roles in materials fabrication. For example, Dillon et al. recently revealed the existence of six distinct grain boundary "complexions" (IGFs and derivative structures) with increasing structural disorder coupled with increasing mobility in doped $\mathrm{Al}_{2} \mathrm{O}_{3}$ (Dillon et al., 2007). This revelation explains the abnormal grain growth mechanism in this system.

Finally, a related long range scientific goal is to develop quantitative interface complexion (phase) diagrams to represent the stability of IGFs, SAFs, and other interfacial structures (Dillon et al., 2007; Luo, 2008). Such diagrams are useful for designing not only fabrication recipes to use the most appropriate interface structures during processing, but also heat treatment recipes to tune the final interface structures for desired performance properties. 
The necessity of developing grain boundary complexion diagrams is demonstrated by recent research on activated sintering. Studies using $\mathrm{ZnO}-\mathrm{Bi}_{2} \mathrm{O}_{3}$ (Luo et al., 1999) and W-Ni (Luo, et al., 2005; Gupta et al., 2007) as model ceramic and metallic materials showed that nanoscale quasiliquid IGFs form well below the bulk eutectic-solidus temperature, where the bulk liquid is not yet stable. Nonetheless, short-circuit diffusion in these subsolidus quasiliquid IGFs leads to activated sintering in these systems, phenomenologically similar to liquid-phase sintering but able to initiate at $<60 \%$ of $T_{\text {solidus. }}$. Hence bulk phase diagrams are not adequate for designing optimal activated sintering protocols. A recently developed quantitative model can predict grain boundary disordering and related onset activated sintering for five tungsten-based binary alloys (Luo and Shi, 2008). Furthermore, a variety of discrete liquidlike interfacial "phases" can form from the interplay of multiple interfacial interactions and a finite atomic size effect. Related concepts and thermodynamic theories are further elaborated in Luo (2008)).

\section{Premelting dynamics and its manipulation}

As discussed in Secs. III.A.3 and II.C.1, Lifshitz theory has successfully predicted the nature of the surface melting of many materials. Premelting of a material refers to the persistence of a film of its liquid phase at temperatures below the normal melting temperature. Most commonly discussed are the Gibbs-Thomson and colligative or impurity effects. The former is a consequence of a solid phase being convex to its melt phase and thus having a lower freezing point than the bulk, and the latter originates in the lowering of the chemical potential of a solvent in the presence of a solute. All crystals, whether in contact with their vapor phase or with another material, have surfaces where the process of melting is initiated: if there is a layer of liquid at the surface, at temperatures below the bulk melting point $T_{m}$, then there is little need to activate the melting process. The ease with which liquids can be supercooled tells us that the melting process is inherently asymmetrical about the transition point.

Interfacial premelting occurs at the surfaces of solid rare gases, quantum solids, metals, semiconductors, and molecular solids and is characterized by the appearance of an interfacial thin film of liquid that grows in thickness as the bulk melting temperature $T_{m}$ is approached from below (Dash et al., 2006). When interfacial premelting occurs at vapor surfaces, it is referred to as "surface melting;" when it occurs at the interface between a solid and a chemically inert substrate, "interfacial melting;" and when at the interface between two grains of the same material, "grain boundary melting." When films at such solid surfaces diverge at the bulk transition the melting is complete, but where retarded potential effects intervene and attentuate the intermolecular wetting forces the film growth may be blocked and thereby be finite at the bulk transition. This latter circumstance, in which the behavior is discontinuous, is referred to as incomplete melting. Because near the bulk transition we can view these phenomena as wetting transitions [surface melting is a special case of triple point wetting; see Dietrich (1988)], the role of LRIs, and particularly long range van der Waals effects, has a broad context and setting which is particularly relevant to the role of geometry in these volume-volume interactions (Lamoreaux, 2005; Dantchev et al., 2007).

We now consider the dynamics of premelted liquid driven by variations in temperature and/or composition. Although the physics of the surface and that of the bulk are most often taught and studied in isolation, it is the confluence of dimensionality and phase behavior found at the surface that provides a wide-ranging area of exploration in science and engineering. Here we summarize aspects of a recent review (Wettlaufer and Worster, 2006).

Two aspects of surfaces familiar to those working in materials are wetting phenomena and thermophoretic or Marangoni flows. Important and present in many settings, premelting dynamics makes some contact with these phenomena, but it is distinct in its origin and consequences. The fluid dynamics of interfacially premelted fluid (the focus here) would not exist in the absence of interfaces, but it requires neither contact lines nor gradients in the coefficients of surface energy.

During complete interfacial premelting under long range forces that are of a power-law form, the film thickness $d$ is related to the temperature $T$ through $d=\lambda\left(T_{m}\right.$ $-T)^{-1 / \nu}$, in which $\lambda$ and $\nu$ are positive constants, the latter is an integer that depends on the nature of the interactions driving melting of the system. The dynamical consequences emerge from the fact that the LRIs provide the field energy per molecule that shifts the equilibrium domain of the liquid phase into the solid region of the bulk phase diagram (Dash et al., 2006; Wettlaufer and Worster, 2006). For an interfacial film of thickness $d$, the shift is then written as

$$
\mu_{d}(T, p, d)=\mu_{l}(T, p)+U^{\prime}(d)=\mu_{S}(T, p),
$$

where $\mu_{l}(T, p)$ and $\mu_{S}(T, p)$ are the bulk chemical potentials of the liquid and solid, respectively, and $U^{\prime}(d)$ is the derivative, with respect to $d$, of the underlying effective interfacial free energy $U(d)$ which itself depends on the nature of the intermolecular interactions in the system. For example, in the case of nonretarded vdW-Ld forces, a phenomenological description is given by

$$
U^{\prime}(d)=\frac{2 \Delta \gamma \sigma^{2}}{\rho_{l} d^{3}}
$$

where $\sigma$ is of order a molecular length, $\rho_{l}$ is the liquid density, and $\Delta \gamma$ is the difference between the interfacial energy of the dry interface between the solid phase and the third phase, be it the vapor, a wall, or a different orientation of the solid phase. The coefficient in Eq. (27) is related to the Hamaker coefficient $\tilde{A}$ by $\tilde{A} / 12 \pi$ $=\Delta \gamma \sigma^{2}$. Thus we see that interfacial melting occurs when $\tilde{A}<0$, so that there is a force of repulsion-disjoining pressure or thermomolecular pressure-between the 
media bounding the liquid film, written here for nonretarded vdW-Ld forces. When the external pressure applied to the third phase, such as a wall, $p_{w}$ equal to that applied to the solid $p_{S}$ balances the thermomolecular pressure $p_{T}=-\tilde{A} / 6 \pi d^{3}$, then $p_{w}=p_{S}=p_{T}+p_{l}$, where the hydrodynamic pressure is $p_{l}$. Combining this with the Gibbs-Duhem relationship, which can be applied on each side of a solid-melt interface, allows one to show in general that when the system is in equilibrium at temperature $T$ then

$$
p_{S}-p_{l}=p_{T}=\frac{\rho_{S} q_{m}}{T_{m}}\left(T_{m}-T\right)
$$

where $\rho_{S}$ is the density of the solid and $q_{m}$ is the latent heat of fusion (Dash et al., 2006; Wettlaufer and Worster, 2006). We imagine fixing the pressure in the bulk solid phase and increasing the temperature toward $T_{m}$ from below; the film thickens, the repulsive thermomolecular pressure decreases, and hence the hydrodynamic pressure increases, driving unfrozen liquid from high to low temperatures. It is important to emphasize that the transport of unfrozen liquid is driven by thermomolecular pressure gradients brought about by temperature gradients and not capillarity.

We discuss a number of applications below but also refer the interested reader to Dash et al. (2006) and Wettlaufer and Worster (2006) for a more thorough treatment.

To enhance the ready sintering of any material it has long been known empirically that to bring the system close to its melting point is advantageous. We now understand that this is due to the enhanced liquidity of the system due to effects of premelting.

A temperature gradient parallel to the surface is responsible for a gradient in the film thickness and thus a thermomolecular pressure gradient. If the film is sufficiently thick, it can be treated as a thin viscous fluid and its flow described by lubrication theory. The effect can be responsible for trapping particles at a bulk solidification front (Rempel and Worster, 2001) and, if the temperature gradient is maintained, the particle within the solid will continue to move. Indeed, a simple analysis reveals that the force on the particle can be written in a manner directly analogous to Archimedean buoyancy $\vec{F}_{B}=-m_{S} \vec{G}$. Here $m_{S}$ is the mass of solid displaced by the particle against which it premelts; $\vec{G}=\vec{\nabla}(\Delta \mu)$ is the gradient in the generalized departure of the system from bulk coexistence $\Delta \mu \propto T_{m}-T$, which is determined here by a departure of temperature from the bulk melting temperature. This is referred to as thermodynamic buoyancy (Rempel et al., 2001). As an example, a particle of a micron in radius moving under the influence of a very weak temperature gradient $(0.025 \mathrm{~K} / \mathrm{m})$ has $\vec{G}$ $\approx 3 \vec{g}$ it moves at about $1 \mu \mathrm{m} /$ year for a nanometer-scale film thickness. From the materials standpoint, we can control the temperature and temperature gradient of many systems accurately and over a wide range. Hence, there is a potential to redistribute particles included in a

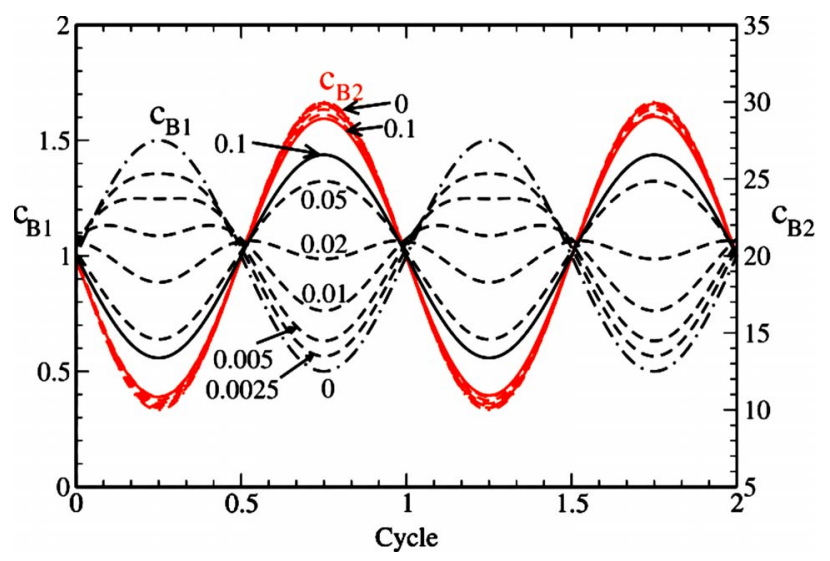

FIG. 24. (Color online) The time evolution of two periodic profiles of bulk (salt concentration in the solid plus liquid system) solute fields deposited on a polycrystalline ice sheet asynchronously (Rempel et al., 2002). The initial profiles are labeled 0 . The units are dimensionless, with the horizontal axis being the distance along the sample, but the essential point is that the major species $\mathrm{c}_{\mathrm{B} 2}$ (solid) controls the liquid fraction and it drives the minor species $\mathrm{c}_{\mathrm{B} 1}$ (dashed) into phase with it on a time scale sufficiently rapidly that its original profile is unchanged.

host solid using premelting dynamics. The idea is to ensure that they enter the host solid through quenching and then redistribute them by manipulation of the temperature field of the system.

The transport properties of unfrozen water in ice polycrystals bears strongly on the redistribution of paleoclimate records, retrieved from the ice cores, that are redistributed by the premelted water (Dash et al., 2006). In fact, as mentioned, any physical or biological process relying on the presence of the liquid phase will be strongly influenced by grain boundary melting because the surface area of a polycrystal is dominated by grain boundary interfaces. Soluble species reside principally in the liquid phase, and their diffusivity is orders of magnitude greater in the liquid than in the solid, hence the fate and evolution of such species is controlled by the volume fraction of liquid in the material. Indeed, a proper homogenization of a two-phase, multicomponent polycrystalline ice system shows a wide variety of coupled processes. For example, a major species controls the (subfreezing) liquid fraction (through the liquidus) and thereby influences the minor species in striking ways (Rempel et al., 2002). In Fig. 24 we show an example of how a major species can be deposited in the sample out of phase with a minor species and after some time the minor species is drawn into phase with the major species. Such a homogenization of multicomponent polycrystalline materials has not been harnessed for the purposes of tailoring materials properties, and thus it seems a unique area ripe for exploration.

Manipulation by freezing rate is also a possible modality of using long ranged interactions to control the morphology and properties of a system. For example, it is possible to treat a colloidal suspension as a "binary alloy" and yet, as shown in Fig. 25, at a solidification 


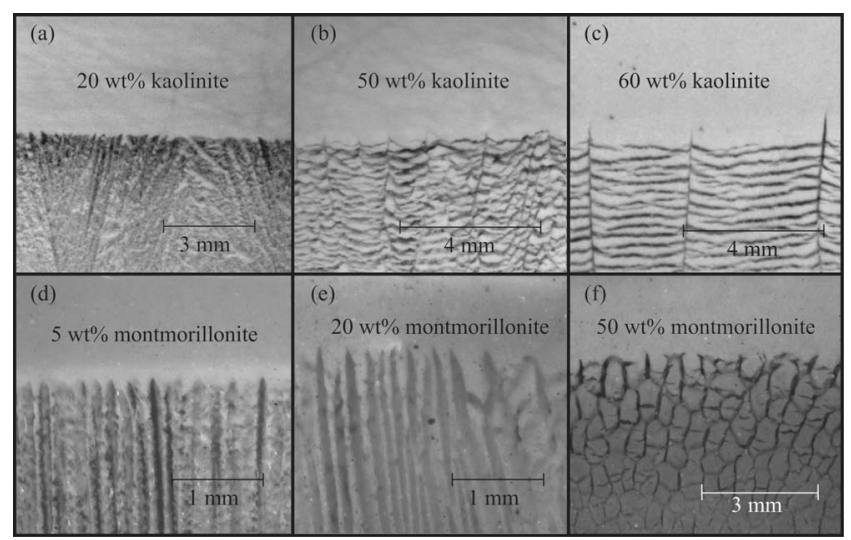

FIG. 25. Colloidal suspensions frozen upward in a cell that is free at its upper end The structure of the ice (dark regions) depends on the conditions of freezing and the particle concentrations. While (a) and (d) exhibit ice dendrites that align colloids in (c) and (f) a polygonal structure forms and there are mixed states between the two geometries initiated by side branching as shown in (e). From Peppin et al., 2006, 2007.

front premelting can conspire to reject particles from the advancing front to create a range of microscopic patterns depending on concentration (Peppin et al., 2007, 2008).

\section{B. Colloids and self-assembly}

\section{Tailored building blocks: From hard spheres to patchy colloids}

The programmable assembly of designer colloidal building blocks with engineered size, shape, and chemical anisotropy is the next frontier in the scientific and technological development of soft materials (van Blaaderen, 2006). Establishing this mastery will enable the assembly of new photonic, electronic, plasmonic, and photovoltaic devices with unparalleled complexity and precision, akin to that achieved in biological systems. As an important first step toward realizing this goal, several synthetic pathways have been recently reported that offer precise control over colloid geometry at the nanoscale (e.g., ellipsoids, triangular plates, cubes, and tetrapods) and microscale (e.g., unary and binary colloidal "molecules"). Despite this impressive geometric control, all of the building blocks shown in Fig. 26 are chemically homogeneous. To date, the ability to spatially encode their surfaces with the desired chemical heterogeneity (or "patchy" structure) remains an elusive goal. Recent simulations demonstrate the transformational impact that this would have on their ability to self-organize into unique structures (Zhang and Glotzer, 2004; van Blaaderen, 2006); see Fig. 27.

To fully harness the potential that spatially and chemically anisotropic colloids ("patchy particles") provide, the following questions must be addressed. First, how do various particle motifs, e.g., janus spheres (particles that consist of oppositely charged hemispheres), ringlike (or striped), or distinct patches influence colloidal assembly? What role do vdW-Ld, electrostatic, and hydrophobic forces play in controlling their assembly? Finally, how do critical parameters such as patch size and charge density, colloid size, density, volume fraction, and solution conditions affect their ability to assemble into the desired equilibrium (crystalline) and nonequilibrium (gel or glassy) phases?

\section{Synthesis and assembly of designer colloidal building blocks}

Current research efforts focus on moving beyond the traditional systems by enabling the creation of designer colloidal building blocks such as colloidal molecules, janus spheres, and other patchy particle motifs. Myriad particle motifs are envisioned, in which surface chemistry, shape anisotropy, faceting, pattern quantization, and branching are controlled (Glotzer and Solomon, 2007). To date, significant progress has been made toward the realization of many of these motifs. Below, we highlight those assembly routes that yield chemically heterogeneous (or patchy) colloids.

Granick and colleagues (Hong et al., 2006) recently reported a highly scalable synthetic pathway for creating bipolar janus spheres. Such species, probably the simplest example of patchy colloids, exhibit orientationdependent interactions that go with presentation of like or unlike charges. This heterogeneous interaction landscape promotes the formation of colloidal molecules that are themselves patchy in nature, somewhat akin to globular proteins.

Recently lithographic patterning has been employed to create more complex, patchy colloidal spheres and

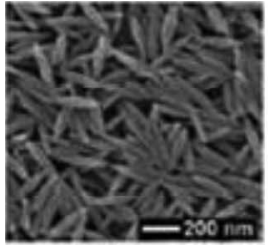

(a)

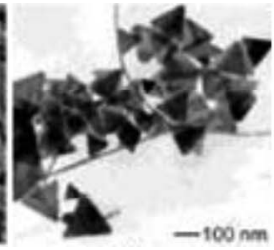

(b)

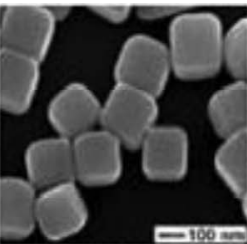

(c)

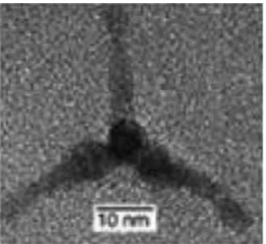

(d)

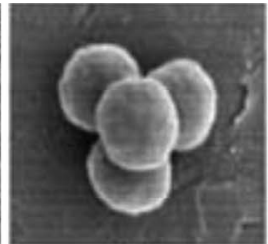

(e)

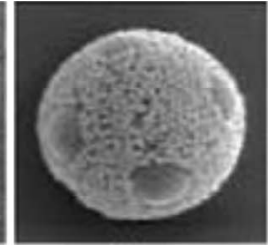

(f)

FIG. 26. Images of synthetic nanoscale and microscale building blocks with controlled architectures: (a) $\mathrm{Fe}_{2} \mathrm{O}_{3}$ ellipsoids. Adapted from Wang, Brandl, et al., 2006. (b) Ag triangular plates. Adapted from Sun, Mayers, et al., 2003. (c) Ag cubes. From Sun and Xia, 2002. (d) CdSe tetrapods. Adapted from Manna et al., 2000. (e) Tetrahedral cluster of polystyrene microspheres (844 nm in diameter) (e.g., unary). From Manoharan et al., 2003. (f) Binary cluster of silica microspheres (2.3 and $0.23 \mu \mathrm{m}$ in diameter, where the number of larger particles equals 8. Adapted from Cho, Yi, et al., 2005. 
(a)

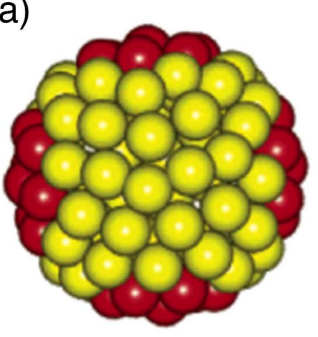

(b)

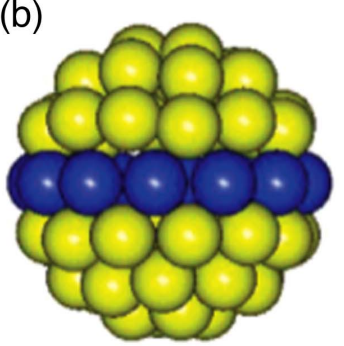

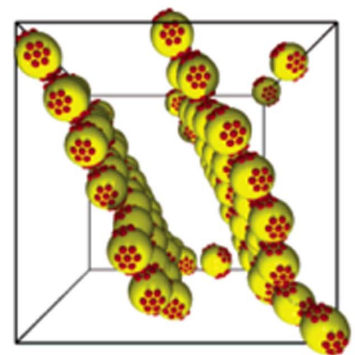

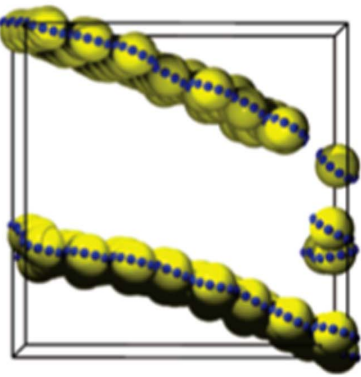

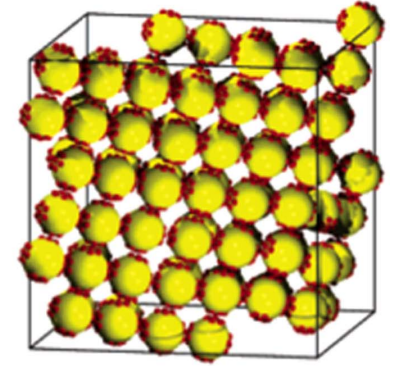

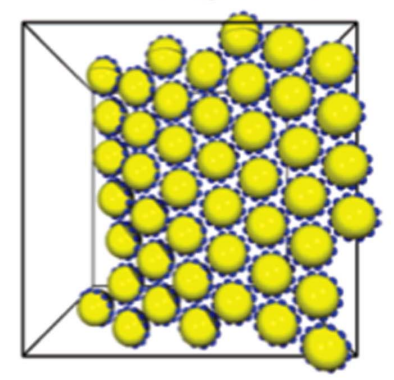

FIG. 27. (Color online) Schematic representations of patchy particles and their predicted equilibrium structures (side and face views) formed via selfassembly: (a) particles with four circular patches assembled at a dimensionless temperature of $k T / \varepsilon=1.0$, and (b) particles with a ringlike patch arranged on an equatorial plane assembled at $k T / \varepsilon=0.5$. Adapted from Zhang and Glotzer, 2004. wires. Möhwald and co-workers (Edwards et al., 2007) created polystyrene microspheres decorated with gold dots with $s p$ valence (Fig. 28). By rendering these patches attractive, one may be able to assemble the spheres into linear particle chains (or strings). Using onwire lithography, Mirkin and co-workers (Qin et al., 2005) created silver-gold metallic nanowires whose spatial composition can be exquisitely tuned (Fig. 28). While neither approach is readily scalable to bulk quantities, they represent the current state of the art in designing chemically heterogeneous building blocks for colloidal assembly.

\section{Challenges and opportunities}

Novel synthetic pathways must be developed that enable the creation of bulk quantities of designer colloidal building blocks of controlled size, shape, and chemical functionality. These pathways must be extended beyond polymeric and silica-based colloids to functional building blocks, such as metals, semiconductors, and complex oxides. A theoretical and computational framework must be developed that is capable of predicting the phase behavior, structure, and assembly of a diverse array of particle motifs. The fundamental understanding of the behavior of designer colloids that would emerge from such predictive tools would provide the synthetic guidelines for creating building blocks that self-assemble into desired equilibrium (crystalline) and nonequilibrium (gel or glassy) phases, while avoiding undesired (or jammed) states.

If the above obstacles can be successfully overcome, "colloids by design" offers enormous potential. Advances in our current synthetic capabilities and fundamental understanding of long range interactions at the nanoscale would open new avenues to engineer crystalline and amorphous phases. For example, the elusive goal of producing a diamond crystal with a lattice constant suitable for photonic band-gap applications could finally be realized. Additionally, colloidal gels could be created with controlled connectivity and elasticity, which may find potential application in the self-assembly of novel Li-ion batteries (Cho et al., 2007) or as inks for direct-write assembly (Smay et al., 2002) of highly efficient solar arrays.
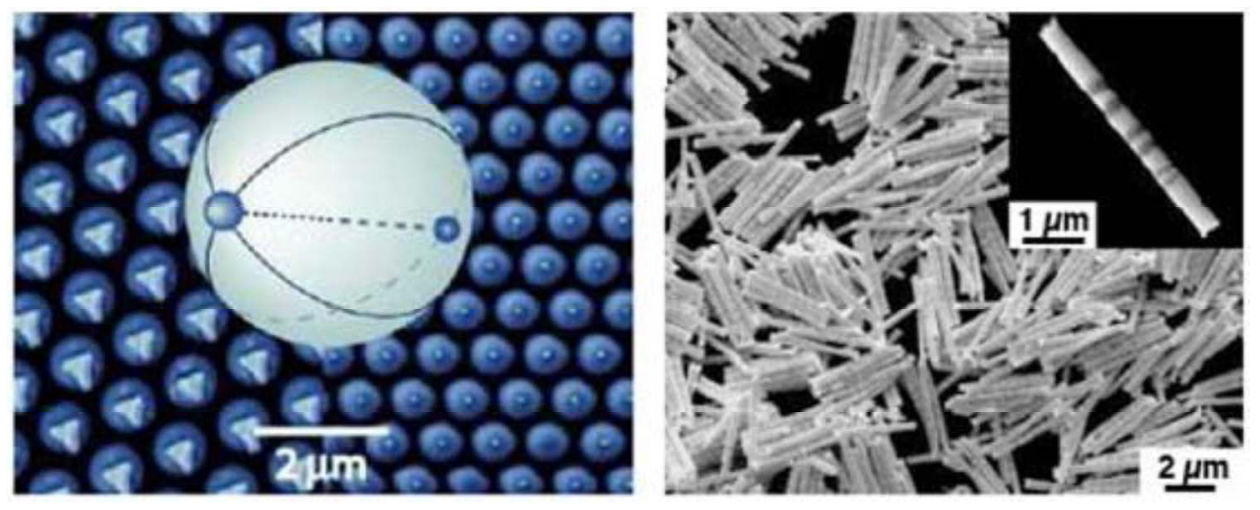

FIG. 28. (Color online) Scanning electron micrographs of lithographically patterned colloids (left) and nanowires (right). Left image: From Edwards et al., 2007. Right image: From Qin, Park et al., 2005. 


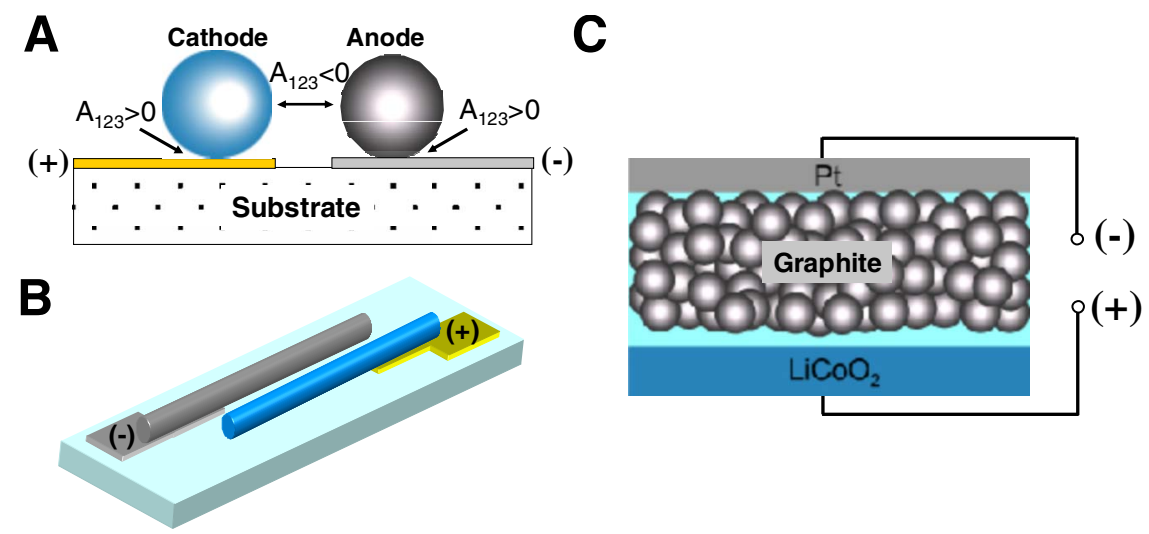

FIG. 29. (Color online) Schemes for self-organization of bipolar electrochemical devices, exemplified using repulsive short range van der Waals-London dispersion (vdW-Ld) interaction (negative Hamaker constant, $A_{123}<0$ ) to form the electrochemical junction while simultaneously using attractive vdW-Ld $\left(A_{121}>0\right)$ to form percolating networks of a single active material and/or to selectively adhere to current collectors $\left(A_{123}>0\right.$ ). (a) Battery formed from a single-particle pair. (b) Nanorod-based batteries. (c) Layered lithium-ion battery using repulsive short range forces to separate $\mathrm{LiCoO}_{2}$ and graphite, while using vdW-Ld attraction to form continuous percolating network of graphite anode.

\section{Self-assembly and emerging device applications}

\section{Electrochemical devices: Li-ion batteries from heterogeneous colloids}

Self-assembly, using intrinsic or applied forces, is a widely accepted concept for the design of novel materials and composites over a wide range of length scales. By contrast, the self-assembly of subassemblies and complete devices has been more challenging, although good examples exist (Colvin et al., 1994; Rueckes et al., 2000; Huang et al., 2001; Whitesides and Boncheva, 2002; Whitesides and Grzybowski, 2002; Gur et al., 2005). Recently it has been demonstrated (Cho et al., 2007) that electrochemical junctions can be formed between conductive device materials using combined vdW-Ld and $A B$ interactions, and in an additional step that the simultaneous implementation of repulsive and attractive interactions can be used to fabricate complete colloidal-scale self-organizing and self-wiring devices. These concepts have been demonstrated in prototype self-organizing lithium rechargeable batteries.

With the advent of nanotechnology, numerous devices have been created that are based on contact junctions between components, including bistable carbon nanotube memory, diodes, light-emitting diodes (LEDs), logic gates, and solar cells (Colvin et al., 1994; Rueckes et al., 2000; Huang et al., 2001; Gur et al., 2005). It is likely that attractive vdW-Ld forces provide the intimate contact between materials that allows electronic transport across these nanoscale Ohmic and $p n$ junctions. In contrast, bipolar electrochemical devices such as batteries, fuel cells, electrochromic displays, and certain types of sensors are based on the separation of electronically conducting electrodes by ionically conducting but electronically insulating electrolytes. Electrode materials of the same type (e.g., cathode or anode) simultaneously need to be continuously connected to their respective current collectors. In principle, the simultaneous control of repulsive and attractive forces can enable the "bottom-up" self-organization of dissimilar colloids into complete bipolar devices as conceptualized in Fig. 29.

Although current theories provide general guidelines for materials selection, the paucity of physical properties data for lithium-active compounds require direct experimentation. Using liquid-cell atomic force microscopy (AFM) and graphite tips [in the form of mesocarbon microbeads (MCMB)], numerous solid compounds and solvents were screened to identify cathode-solventanode combinations between which repulsive interactions exist. Figure 30 shows typical results in which four solvents and five different solids were characterized, and shows a range of interactions from strongly attractive to strongly repulsive. Detailed analysis showed that these interactions cannot be explained on the basis of vdW-Ld forces alone, but rather they indicate a strong and occasionally dominant role of $\mathrm{AB}$ interactions. Using $\mathrm{LiCoO}_{2}$ as the positive and graphite as the negative electrode material, and solvents providing a repulsive interaction (modified to obtain a lithium conducting electrolyte), self-assembling batteries exhibiting reversible Faradaic storage were demonstrated (Fig. 31). This general approach to colloidal-scale self-assembly of heterogeneous colloids could be extended to a range of bipolar device types, and would benefit from improved fundamental understanding of the long range interactions between device materials.

\section{Active electronic devices: Single-walled carbon nanotubes}

Many novel applications of SWCNT have been proposed and demonstrated (Baughman et al., 2002). We mention here only some representative examples in electronics where the control of LRI is likely to play an enabling role.

Semiconducting SWCNT channels have been used to construct field effect transistors. They have some of the highest mobilities and can act as ballistic conductors (Tans et al., 1998; Appenzeller et al., 2002; Javey et al., 

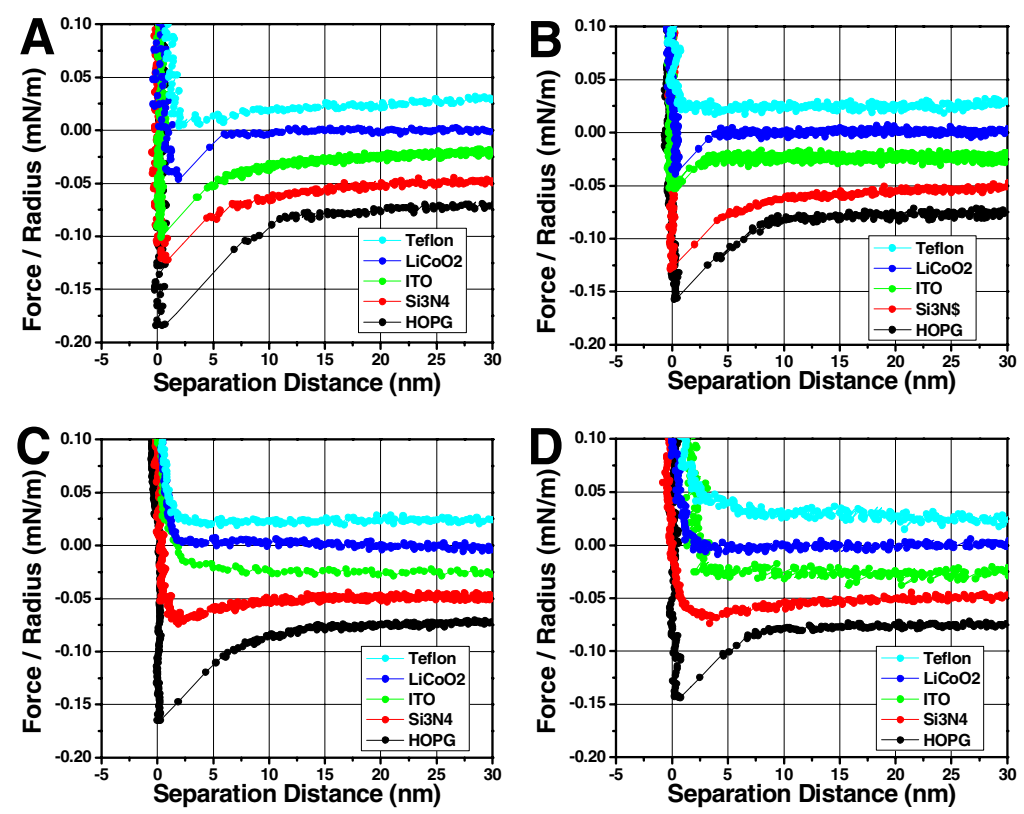

FIG. 30. (Color online) Force curves measured between MCMB probes and five substrates $\left(\mathrm{PTFE}, \mathrm{LiCoO}_{2}, \mathrm{ITO}, \mathrm{Si}_{3} \mathrm{~N}_{4}\right.$, and HOPG) in (a) acetonitrile, (b) m-xylene, (c) ethanol, and (d) methylethyl ketone (MEK; 2-butanone) using liquid-cell atomic force microscope (AFM) show behavior ranging from strong attraction to strong repulsion.

2003). While individual devices with impressive performance and some simple circuits have been demonstrated, considerable progress is required in fabrication, materials, and device design so that these devices can find application as general-purpose circuit elements. Important issues include the development of effective gate dielectric materials, fabrication of good contacts between SWCNT and electrodes, large-scale fabrication, demonstration of high-frequency operability, and ability to dope controllably with $n$ - and $p$-type charge carriers. Heinze et al. (2002) showed that CNT-FET operation can be governed by modulation of the Schottky barrier with the metal contact. Wind et al. (2003) measured performance of CNT-FETs with multiple, individually addressable gate segments, suggesting a transition of switching from Schottky barrier to the nanotube channel. Javey et al. (2003), using Pd contacts in CNT-FET, reduced the Schottky barrier resistance and achieved ballistic transport at room temperature. Guo and coworkers (Guo, Goasguen, et al., 2002; Guo, Lundstrom, and Datta, 2002) provided a theoretical study on the electrostatics of ballistic CNT-FET in one and two dimensions by solving the Poisson equation selfconsistently with carrier statistics. Because of their robustness and small dimensions, carbon nanotubes have proven to be effective in field emission devices, serving as field concentration points on an electrode from which electrons are easily emitted (de Heer et al., 1995). They are being developed for use in field emission displays (Lee et al., 2001).
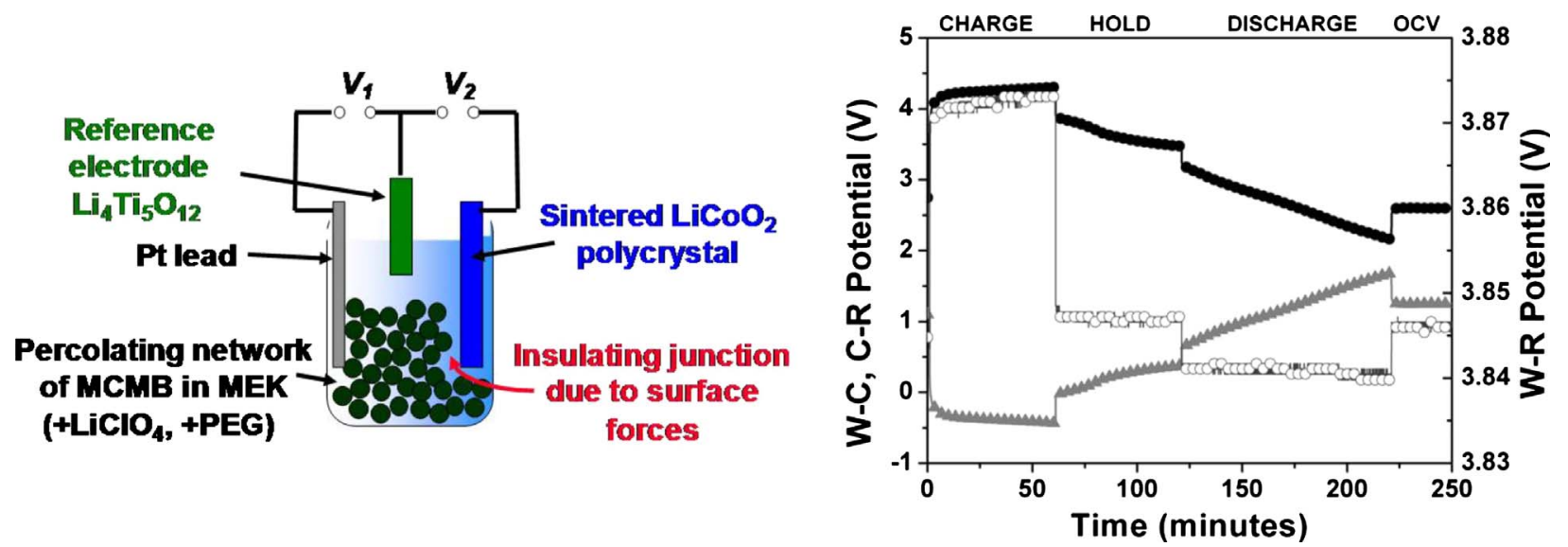

FIG. 31. (Color online) Three-electrode cells using lithium titanate reference electrodes (labeled R) allowed working voltage as well as the potentials at the working $\left(\mathrm{LiCoO}_{2}\right.$ labeled W) and counter (MCMB carbon labeled C) electrodes to be independently measured. Right: Galvanostatic cycling (11th cycle) of a self-organized $\mathrm{LiCoO}_{2}$-graphite rechargeable cell, charging at $100 \mu \mathrm{A}$ and discharging at $-20 \mu \mathrm{A}$ conducted in $\mathrm{MEK}+0.1 \mathrm{M} \mathrm{LiClO}_{4}+1$ wt \% PEG 1500. 


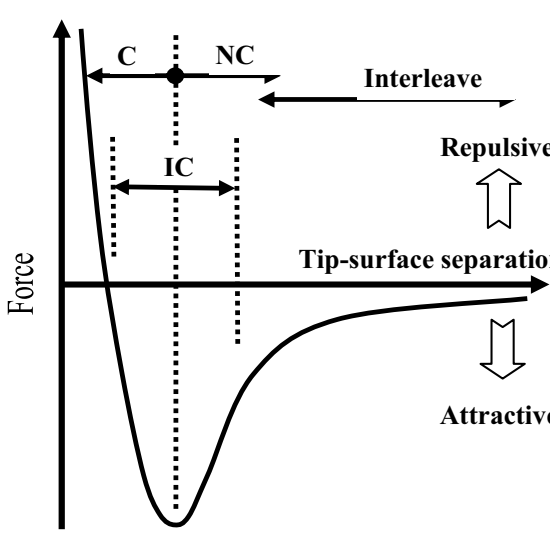

(a)

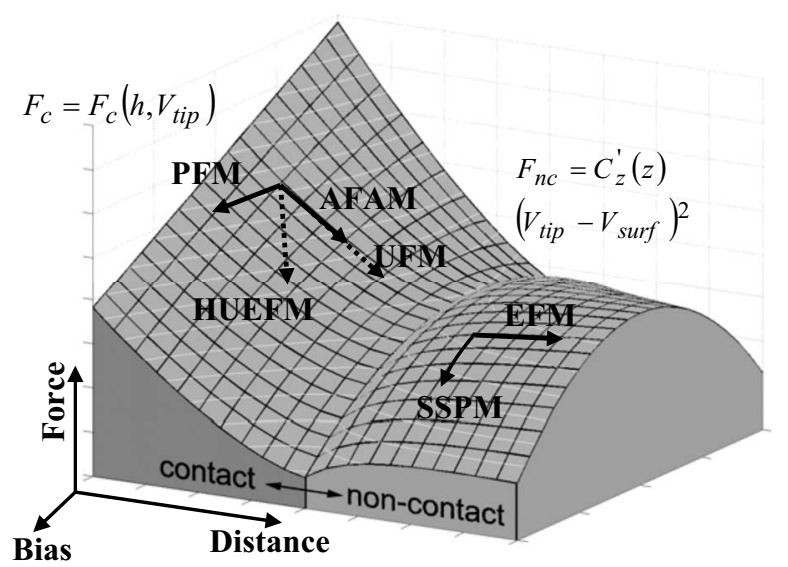

(b)

FIG. 32. The SPM imaging mechanism can be represented as a force distance bias surface. (a) Force-based scanning probe microscopy (SPM) can be conveniently described using a force-distance curve, showing the regimes in which contact (C), noncontact (NC), intermittent contact (IC), and interleave imaging are performed. Also shown are domains of repulsive and attractive tip-surface interactions. (b) Voltage modulation SPMs can be described using force-distance bias surface. In the small-signal limit, signal in techniques such as piezoresponse force microscopy, atomic force acoustic microscopy, electrostatic force microscopy, and Kelvin probe force microscopy is directly related to the derivative in bias or distance direction. Adapted from Kalinin et al., 2007.

SWCNT-based channels expose all their atoms to the environment. This exposure allows use as very sensitive sensors (Qi et al., 2003; Staii et al., 2005; Gruner, 2006). Such devices can be gated efficiently in solutions with possible use as sensors of dissolved species or of the electrochemical environment (Rosenblatt et al., 2002). Rosenblatt et al. fabricated devices that, using electrolyte gating, achieved low contact resistance, excellent gate coupling, and considerably higher mobility than back-gated CNT-FETs. Larrimore et al. (2006) showed that such devices can be used to measure the change in solution electrostatic potential (for given gate voltage) due to the presence of redox-active species in solution.

\section{Nanoscale probes of long range interactions}

\section{Scanning probes}

Scanning probe microscopy (SPM) offers a way to probe LRIs on the nanoscale directly (Hofer et al., 2003; Butt et al., 2005; Foster and Hofer, 2006). In the last decade, SPM-based force spectroscopy and optical tweezers technology have led to new areas of learning, including thermodynamics and kinetics of single-molecule reactions (Ritort, 2006). Given the large number of reviews, we describe here only the general principles of SPM as applied to LRIs, identify some of the challenges, and outline some of the possible pathways for development.

\section{a. The SPM approach}

The essence of an SPM approach is a combination of a local probe bearing a specific aspect of studied functionality [e.g., charged (Nonnemmacher et al., 1991) or chemically functionalized (Noy et al., 1995)] combined with a positioning system that locates it with respect to the studied system, and a detection system that mea- sures forces or currents on or other interactions through the probes, thus linking nanoscale probe-surface interactions to the macroscopic world.

From the perspective of LRI measurement functionality, there are two parameters that can be controlled in an SPM experiment, namely, tip-surface separation and probe bias. Detected are the probe current and force components acting on the probe. Hence, the SPM imaging mechanism can be represented as force-distance-bias surface $\mathbf{F}_{c}=\mathbf{F}_{c}\left(h, V_{\text {tip }}, \mu\right)$, where $h$ is the tip-surface separation (for noncontact methods) or indentation depth (for contact), $V_{\text {tip }}$ is the probe bias, and $\mu$ are parameters describing chemical functionality of the probe (Fig. 32).

One of the primary challenges in SPM probing of the LRI is the decoupling of multiple interactions simultaneously present in the tip-surface junction that all contribute to the forces, $\mathbf{F}_{c}$. One approach to measurement of LRI is based on measurement force at several separations from the surface. The alternative is offered by modulated methods in which a specific functionality of the probe is modulated and the oscillatory response is detected. This allows both probing a subset of tipsurface interactions (e.g., oscillating electrostatic potential does not affect VdW forces), minimizing noise level utilizing resonance enhancement, and probing linear response dynamics though measurement of both amplitude and phase of response. Practically, only some aspects of probe functionality can be modulated at rates of $>1 \mathrm{kHz}$, required for imaging. These include position $h$ (e.g., acoustic driving) or force $\mathbf{F}_{c}$ (e.g., magnetic driving) and electrical bias $V_{\text {tip }}$. Chemical functionality, hydrophobicity, and other chemical functionalities $\mu$ do not offer obvious universal strategies for modulation, even though optically and bias-induced transformations provide some possibilities. Below we discuss SPM techniques based on whether the separation between different interactions is achieved through force-based 
measurements or voltage modulated measurements.

$i$. Direct force measurements. The most straightforward application of SFM to probe long range interaction forces is direct measurements of static (dc) force between functionalized probe and the surface using the cantilever position detection. The experimentally achievable force sensitivity level is of the order of 1-10 $\mathrm{pN}$, sufficient to probe a single hydrogen bond (Rief et al., 1997).

One of the most exciting examples of this approach is molecular unfolding spectroscopy, in which forcedistance curves obtained at different rates contain information on thermodynamics and kinetics of forceinduced reaction on a single molecule level. Alternatively, the force-distance curves can be used to determine local adhesion, indentation modulus, or long range electrostatic interactions. Chemical functionalization of the probe allows controlling the nature of tipsurface interaction so as to probe hydrogen bonding and polar interactions. Butt et al. (2005) provided an indepth account of force-based studies of materials.

The direct force measurements at each point bring a dual challenge of large data acquisition times and relatively low pixel density, and the necessity to analyze the large 3D arrays of data to extract relevant parameters that can be plotted as 2D maps. Existing approaches for measuring LRIs are based on the decoupling topography from measured force components either through (a) height variation, (b) use of force-modulation approaches, (c) methods using fast spectroscopy, or (d) detection through complementary mechanical degrees of freedom.

Examples of the height variation approach are wellknown double-pass or interleave modes. In these, the first AFM scan is used to determine the position of the surface, i.e., the condition at which the measured signal $R\left(h, V_{0}\right)=R_{0}$, where $R_{0}$ is a set-point value. The feedback signal $R$ can be static deflection for contact AFM, oscillation amplitude for amplitude-based detection signal, or frequency shift for frequency-tracking methods. The second scan is performed to determine interactions at different distance and bias conditions to measure $R$ $=R\left(h+\delta, V_{1}\right)$. As a typical example, magnetic and electrostatic force microscopies utilize the fact that these forces are relatively long range and weak compared to the vdW interactions. Hence, once the position of the surface has been determined, force measurement at significant $(\delta=10-500 \mathrm{~nm})$ separations yield magnetic (if the probe is magnetized) or electrostatic (if the probe is biased) force components.

The alternative approach to force detection is based on ac modulation approaches, e.g., atomic force acoustic microscopy, force modulation, and similar methods. In these, the condition of $R\left(h, V_{0}\right)=R_{0}$ is used to determine the position of the surface, and modulation of the probe height or bias is used to obtain additional information on the distance or bias derivative of the force-distancebias surface. For example, in the small-signal approximation, the AFAM signal is related to $(\partial h / \partial F)_{V=\text { const }}$.
This decoupling can be performed dynamically by response phase and amplitude in AFM phase imaging (amplitude yield information on topography and phase yield information on elasticity and adhesion).

A number of approaches for LRI detection are based on data processing beyond simple lock-in or PLL detection. For example, the use of the functional agent coupled to the probe with a flexible linker, combined with separate detection of the signal from top and bottom of the trajectory, forms the basis of molecular recognition imaging (Raab et al., 1999). A number of methods have been developed to sample a larger region of the force-distance phase space of the system beyond small-signal approximation, e.g., pulsed force mode (Miyatani et al., 1997).

Finally, different interactions can be decoupled using flexural and torsional degrees of freedom of the probe, with normal force mapping the topography, while friction forces are sensitive to adhesion and chemical interactions. In lateral force microscopy (Mate et al., 1987), the topography is detected from the normal force signal, while the friction force detected from the lateral signal provides information on the short range tip-surface interactions that are sensitive to local chemical composition, molecular orientation, etc. Recent development of the harmonic detection method (Sahin et al., 2007) allows reconstruction of tip-surface interactions based on decoupling between torsional and lateral oscillations.

ii. Voltage modulation approaches. An efficient approach to decoupling vdW-Ld and electrostatic components is based on the use of voltage modulation. Electrically modulated SPMs include Kelvin probe force microscopy (KPFM) with amplitude (measured force) and frequency (measures force gradient) feedback, and piezoresponse force microscopy. Applications of these modes are well known in areas as diversified as local work function imaging (Henning et al., 1995), mapping electrostatic potential in operational devices (Shikler et al., 1999), and sub-10-nm structural imaging of calcified tissues based on piezoelectricity (Kalinin et al., 2005). The spatial resolution and sensitivity of these methods are dictated by environmental limitations and the nature of tip-surface interactions to $\sim 1 \mathrm{mV}$ and $\sim 30 \mathrm{~nm}$ for KPFM and $1 \mathrm{pm} / \mathrm{V}$ and $\sim 5 \mathrm{~nm}$ for PFM.

Progress in areas such as high-resolution imaging, probing macromolecular transformations, or cellular and subcellular electrophysiology necessitates implementation of electrically modulated SPM in liquid conductive environments. The key task here is the capability to control dc and ac electric potential on small length scales. Experimentally, it has been shown that the use of sufficiently high ac frequencies accessed through direct imaging or frequency mixing down conversion allows probing ac behavior (ac field is localized) (Lynch et al., 2006; Rodriguez et al., 2006). At the same time, dc fields are not localized in most solvents (Fig. 33) (Rodriguez, Callahan, et al., 2007). The development of insulated and shielded probes (Rosner and van der Weide, 2000; Frederix et al., 2005) offers a pathway to future progress. 


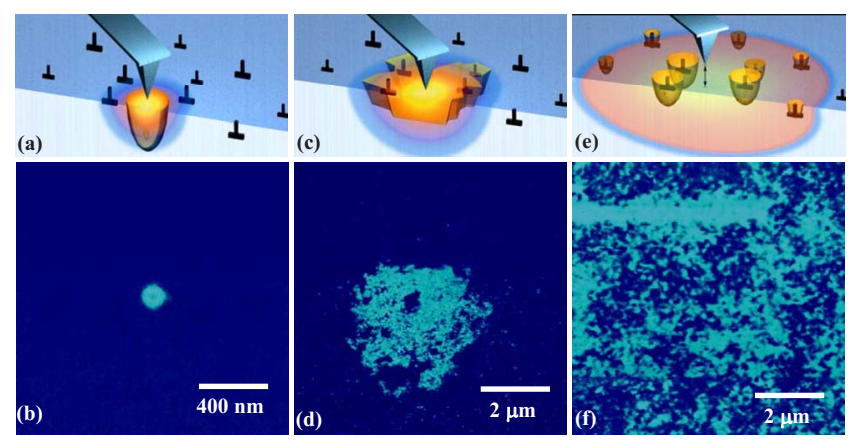

FIG. 33. (Color online) Three cases of electrically modulated SPM in liquid conductive environments. Schematics [(a),(c),(e)] and PFM phase images [(b),(d),(f)] of switching in (a),(b) local, (c),(d) fractal, and (e),(f) nonlocal cases. The size of switched region illustrates the spatial extent of the electric field. The localized switching was observed only in ambient environment, while fractal-like clusters are observed in methanol and isopropanol. In aqueous solutions and DI water, only nonlocal switching is observed, indicative of nonlocalized dc electric field. From Rodriquez et al., 2007.

iii. Functional probes. In the last several years, a number of approaches for imaging have emerged based on probes carrying more complex local functionalities, including field effect transistors (Park et al., 2004) and electrochemical probes, among others. While the field is still nascent, there is tremendous potential for progress.

iv. Probing dissipative dynamics. Understanding long range interactions ideally requires probing not only the conservative, but also the dissipative part of the force. SFM offers a natural approach to probing local dissipation by comparing the quality factor of the cantilever interacting with the surface and away from the surface. Assuming that the internal dissipation of a cantilever does not change and that environmental dissipation changes only insignificantly, changes in the $Q$ factor will be due only to the tip-surface dissipation. Simple estimates suggest that detection limits set by the thermomechanical noise are of the order of $3 \times 10^{-17} \mathrm{~W}$ (the experimentally achieved limit is $\sim 0.03-0.1 \mathrm{pW}$ ) (Proksch et al., 1999). However, until recently dissipation probing in SPMs has been limited. Indeed, cantilever dynamics in the vicinity of resonance in the simplest harmonic oscillator approximation is described by three parameters: resonance amplitude, resonance frequency, and $Q$ factor. Experimentally, SFM based on sinusoidal modulations, which are now the vast majority of experimental platforms, measure only two independent parameters, amplitude and phase in lock-in detection, and resonance frequency and amplitude for phase-locked loop-based frequency-tracking methods. Hence, dissipation is not addressed directly. Practically, with a single-frequency modulation, dissipation can be determined if the driving force acting on the system is known. This provides an additional constraint on the signal, an assumption implicitly used by Cleveland et al. (1998) and Garcia (Tamayo and Garcia, 1998). In frequency-tracking methods (Albrecht et al., 1991) the presence of an additional constraint implies that the response amplitude is inversely proportional to the $Q$ factor. These approaches have been implemented by several groups to study magnetic dissipation (Grütter et al., 1997; Proksch et al., 1999), electrical dissipation (Denk and Pohl, 1991), and mechanical dissipation on atomic (Kantorovich and Trevethan, 2004) and molecular levels (Farrell et al., 2005).

This approximation is applicable in a limited number of cases, such as magnetic driving (if the sample is paramagnetic) or acoustic driving with a flat (no dispersion) transfer function. Even with acoustic driving by the piezo element, adopted in most SPMs, the nonidealities in the transfer function of the piezodriver lead to the qualitatively incorrect results in, for example, MFM dissipation studies. For methods based on electric excitation, the relationship between the driving voltage and the local force is position dependent, and this dependence is convoluted with cantilever response function, and the two cannot be distinguished by single-frequency measurements. Simple frequency sweeps or ring-down measurements are usually time consuming, and thus have only limited applicability to imaging applications. Recently the advent of multiple-modulation methods such as dual ac (Rodriguez, Callahan, et al., 2007) and band excitation (Jesse et al., 2007) that allow simultaneous probing of finite region of Fourier space offer a potential path forward.

\section{b. Future developments}

One of the least understood and least used aspects of nanoscale is the conversion between electrical and mechanical phenomena beyond simple capacitive forces. Electromechanical coupling occurs everywhere in biological systems, in processes from hearing to motion to cardiac activity; it also occurs in soft condensed matter systems such as polyelectrolytes, redox-active molecules, ferroelectric polymers, etc. However, electromechanical properties are traditionally difficult to access even in macroscopic systems due to the smallness of corresponding coupling coefficients. On the nanoscale, even thinfilm measurements have become possible only with the introduction of double-beam interferometer systems in the 1990s. This is in stark contrast to the mechanical and transport measurements that evolved continuously from macroscale to nanoscale. SPM methods combining high field localization and sensitivity to extremely small mechanical response offer a unique capability to study electromechanical coupling on the nanoscale. While introduced only in 1996 for ferroelectrics, these methods have become the mainstay for characterization of ferroelectric materials. Their applicability to piezoelectric biopolymers and III-V nitrides and imaging in liquid has recently been demonstrated. These materials will open the pathway for probing and controlling electromechanical conversion on a single-molecule level and harnessing these for device applications (Fig. 34). 


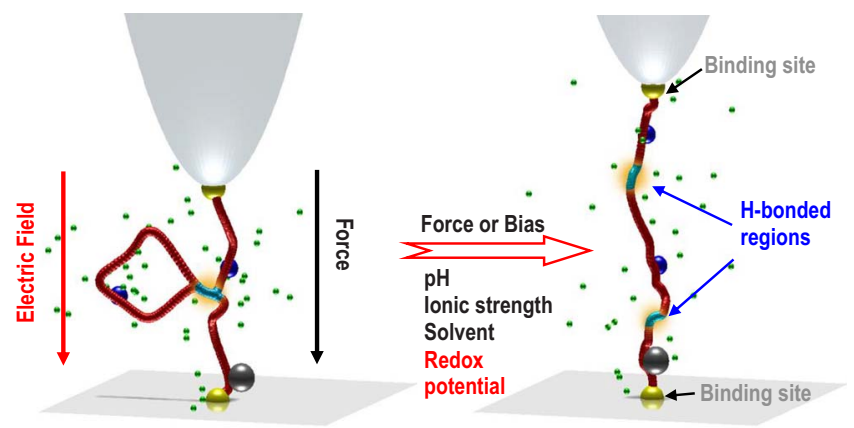

(a)

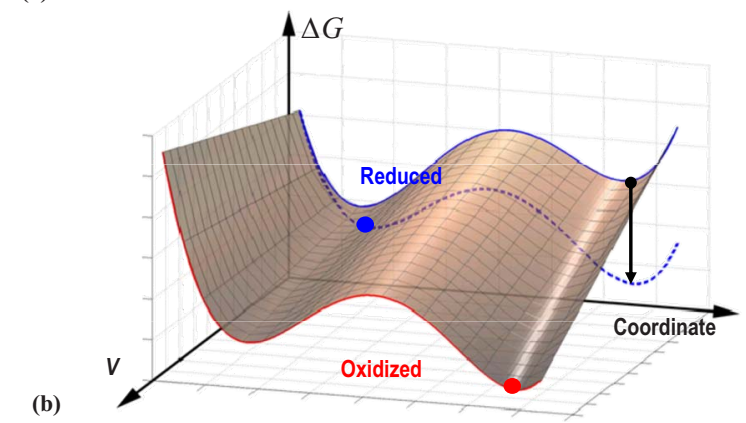

FIG. 34. (Color online) Probing and controlling electromechanical conversion of a single molecule level. (a) Schematic of molecular transformation change in electric field in the tipsurface junction. (b) Corresponding force-bias-distance surface. The redox potential of the molecule is expected to depend on the fore acting on the molecule.

\section{Scattering probes}

a. X-ray scattering

The worldwide availability of synchrotron $\mathrm{x}$-ray and neutron scattering user facilities (Brown et al., 2006) has made it routinely possible to determine the structure and dynamics of both free and buried interfaces at subangstrom to millimeter length scales (Fenter and Sturchio, 2004) and femtosecond to millisecond time scales (Rheinstädter et al., 2006). Advances in computational theory, algorithms, and processing power are beginning to permit quantitative comparison of atomic and molecular interactions in condensed phases over similar length and time scales (Cygan and Kubicki, 2001; Schoen and Klapp, 2007). This convergence of capabilities offers unprecedented opportunities for exploring LRIs at the nanoscale for materials science and engineering.

Electromagnetic radiation is elastically or inelastically scattered by the electrons surrounding atomic nuclei, and the penetrating power of $\mathrm{x}$ rays has long been applied in imaging biological and materials structures. The physics of interaction of electromagnetic radiation with atoms is precisely known, permitting crystalline structures to be determined with extreme precision. Synchrotron x-ray sources provide highly intense beams, the energies and polarizations of which are highly tunable. These enhancements over conventional x-ray sources permit a wide range of unique studies of condensed matter, as recently reviewed for earth science applications by Fenter et al. (2002), and references therein. The high intensities and wide range of wavelengths available from synchrotron sources permit determination of local ordering, even in glasses and other amorphous or weakly crystalline materials (Egami, 2004, 2007). X-ray absorption and fluorescence provide direct identification of the specific elements interacting with the beam, and methods such as absorption fine structure, near-edge, and photoelectron spectroscopy can be used to determine the valence state of the target element and its nearestneighbor distributions and elemental compositions (Evans et al., 2003; Glover and Chantler, 2007; Wada et al., 2007). 3D tomographic imaging of structures and elemental distributions within condensed matter is now routinely performed at synchrotron light sources (Sutton et al., 2002), even at high temperatures and pressures (Wang et al., 2005).

Synchrotron x-ray methods for probing elemental distributions at interfaces have been highly developed in recent years. Grazing-incidence small-angle scattering and absorption fine structure can give precise information on the bonding configuration of sorbed ions and the distribution and orientation of nanoparticles on solid substrates (Waychunas, 2002; Waychunas et al., 2005; Saint-Lager et al., 2007). Crystal truncation rod studies can provide three-dimensional information, at subangstrom resolution, on the relaxation of crystalline surfaces and the distributions of water and ions in liquid electrolytes at the crystal-water interface (Catalano et al., 2007; Lee et al., 2007; Zhang et al., 2007). Some examples for cations on the rutile (110) surface are described in Sec. III.C.3. Resonant anomalous reflectivity can probe a wide length scale on either side of a crystal/ fluid interface and can give information on the distribution of elements not specifically oriented relative to the underlying crystal structure (Fenter et al., 2007; Park and Fenter, 2007). Reflectivity studies have also revealed the interaction of water with hydrophobic surfaces (Poynor et al., 2006). X-ray standing wave studies can probe the distribution of fluorescent trace elements at interfaces with crystalline phases, also at sub-angstrom resolution, and distinguish the ordered fraction of the total distribution of the trace element, relative to the underlying crystal structure (Zhang et al., 2004). Buried interfaces between dense solids have also been probed by nonspecular scattering and absorption spectroscopy (Lutzenkirchen-Hecht et al., 2007).

\section{b. Neutron scattering}

The dependence of x-ray scattering on atomic number presents problems for (a) probing deeply into dense matter, especially if the material is composed of heavy elements; and (b) detection of elements lighter than carbon. Neutrons, on the other hand, are scattered by atomic nuclei, which occupy an exceedingly small volume of even the densest phases, such that neutrons can penetrate many centimeters of dense matter. This makes high pressure-temperature studies readily possible, and important advances in deep Earth petrology and materials are being made at neutron diffraction facilities $(\mathrm{Su}-$ zuki et al., 2001; Matthies et al., 2005). The coherent scat- 
tering length can be positive or negative, depending on whether the interaction with the nucleus is attractive or repulsive. Furthermore, the scattering lengths are a complex function of the atomic weight (rather than atomic number), and thus different isotopes of the same element interact differently with neutron beams, and the light elements interact strongly with neutrons, making detection independent of atomic number (Bee, 1988; Kreitmeir et al., 2007). Hydrogen, which is nearly impossible to detect by $\mathrm{x}$-ray scattering, has a coherent neutron scattering length comparable to heavier elements, and a very large incoherent scattering length, useful for probing the dynamics of hydrogen-bearing compounds. The coherent scattering lengths of hydrogen and deuterium are opposite in sign, making many unique experiments possible. Wenk (2006) provided a recent and thorough review of neutron scattering applications in the Earth sciences, highlighting techniques readily applicable to materials science.

Neutron diffraction is complementary to x-ray diffraction. Light elements as well as heavy elements are readily detected (Goncharenko, 2005; Kim et al., 2007), and isotope substitution can be used to isolate specific substructures within complex crystals and weakly crystalline materials. A unique aspect of neutron scattering is its sensitivity to magnetic substructures within solid phases (Hayward et al., 2005; Kimber et al., 2006; Apetrei et al., 2007). Isotope substitution can even be used to determine the hydration and complexation structures of ions in homogeneous liquids (Enderby, 1995; Neilson et al., 2001; Muenter et al., 2007). Neutron wide-angle, small-angle, and ultrasmall-angle scattering has been extensively used to identify structures (scattering density contrasts) in complex fluids, polymer blends, and solid phases angstrom to millimeter length scales. This technique is also widely employed in determining surface fractals, particle size distributions, and pore-filling characteristics of ceramics, metals, and mesoporous materials (Ficker et al., 2007; Kaewsaiha et al., 2007; Rother et al., 2007).

Another unique feature of neutron scattering is the strong incoherent scattering of hydrogen which results from the gain or loss of energy of incident neutrons interacting with the same hydrogen nucleus within a sample at different times. Neutron inelastic (INS), quasielastic (QENS), and spin-echo (NSE) spectroscopies can be used to probe dynamics ranging from vibrational densities of states to translational and diffusional dynamics of water molecules and other hydrogen-bearing molecules in bulk systems and as surface coatings at open or buried interfaces (Cole et al., 2006). QENS has been extensively employed in studies of the dynamics of bulk water (Teixeira et al., 1985) and water confined within nanoscale pores in a variety of inorganic substrates, predominantly various silica matrices, such as Vycor and Gelsil glass and MCM zeolites (BellissentFunel et al., 1993, 1995; Takamuku et al., 1997; Takahara et al., 1999, 2005; Zanotti et al., 1999; Crupi et al., 2002a, 2002b; Mansour et al., 2002; Faraone, Liu, Mou, Shih, Brown, et al., 2003; Faraone, Liu, Mou, Shih, Copley,
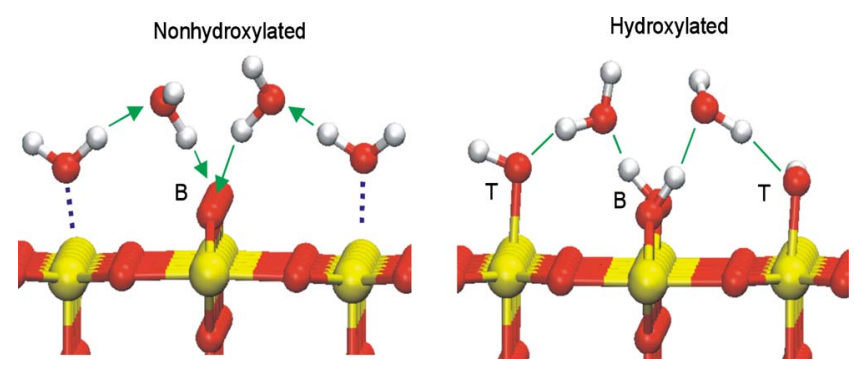

FIG. 35. (Color online) Hydrogen-bonding configuration of chemisorbed $L_{1}$ water molecules which sit atop bare fivecoordinated metal atoms at the $\langle 110\rangle$ surface of rutile $\left(\alpha-\mathrm{TiO}_{2}\right)$ and cassiterite $\left(\alpha-\mathrm{SnO}_{2}\right)$ as either intact water molecules (left) or dissociated hydroxyl groups (right) at "terminal" $(T)$ and "bridging" $(B)$ surface oxygen sites, and physisorbed $L_{2}$ water molecules hydrogen bonded to the surface groups.

and Chen, 2003; Liu et al., 2004) and thin films on the surfaces of metal-oxide nanoparticles (Mamontov, 2004, 2005a, 2005b). These studies demonstrated that water in such nanoscale environments is very different from bulk water, exhibiting liquidlike dynamic motions at temperatures far below the freezing temperature of bulk water.

Mamontov et al. (2007) showed that water sorbed on rutile-structured $\mathrm{TiO}_{2}$ and $\mathrm{SnO}_{2}$ nanoparticles is structurally similar to bulk water in contact with the macroscopic $\langle 110\rangle$ surfaces of these phases. As shown in Fig. 35 the first structural layer $\left(L_{1}\right)$ is composed of water molecules chemisorbed strongly to undercoordinated metal atoms at the crystal surface, and the second structural layer $\left(L_{2}\right)$ consists of water molecules strongly hydrogen-bonded to $L_{1}$ and bridging surface oxygen atoms. Coupling QENS and MD, Mamontov et al. (2007, 2008) determined the rotational and translational dynamics of these and more loosely bound $L_{3}$ water molecules and uniquely assign the dynamic features to (a) hindered rotations of water molecules within their hydrogen-bonded cages in all layers, with characteristic relaxation times $(\tau)$ in the $1-10 \mathrm{ps}$ range; (b) coupled rotational-translational motions of water molecules with undersaturated hydrogen-bonding environments in $L_{3}$ (10-100 ps range); and (c) translational jumps of $L_{2}$ water molecules into $L_{3}$ (100-1000 ps range). QENS is sensitive only to hydrogen dynamics, but the MD simulations capture both hydrogen and oxygen dynamics in water molecules. As shown in Fig. 36, this enabled unique identification of the origin of the slow translational component, and demonstration that $L_{1}$ water molecules do not undergo translations on the time scale detectable by QENS. The excellent agreement between QENS and MD determinations of the rotational and coupled rotation-translation components is shown in Fig. 37.

The series of studies of water at metal-oxide surfaces summarized in the Secs. III.C.3 and IV.D.2 demonstrates the power of combining synchrotron $\mathrm{x}$-ray reflectometry with inelastic neutron scattering and ab initio optimized classical MD simulations to uniquely determine the structure and dynamics of interfacial water at the ang- 


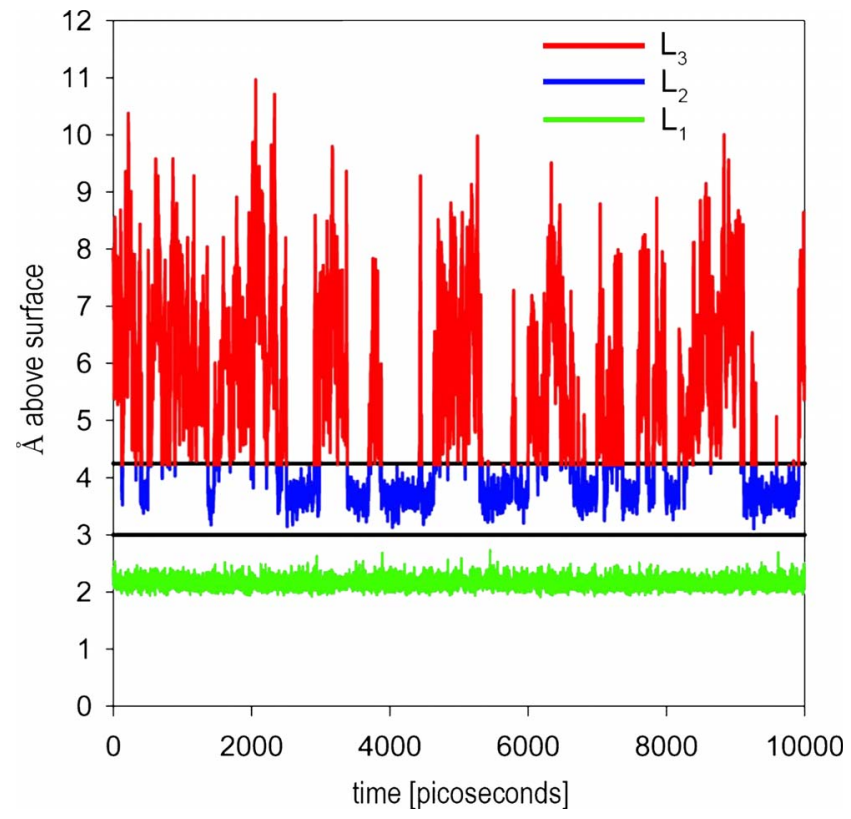

FIG. 36. (Color online) MD simulation results of oxygen atom trajectories above the rutile $\langle 110\rangle$ surface for water molecules that originated in $L_{1}$ and $L_{2}$, as a function of time. Adapted from Mamontov et al., 2008.

strom and picosecond length and time scales. The computational approaches treat LRIs (all of which arise from interatomic and intra-atomic electron interactions) at varying degrees of rigor. It has been demonstrated in this and many other systems that high-level treatment of LRIs at the electronic structure level is only practical for

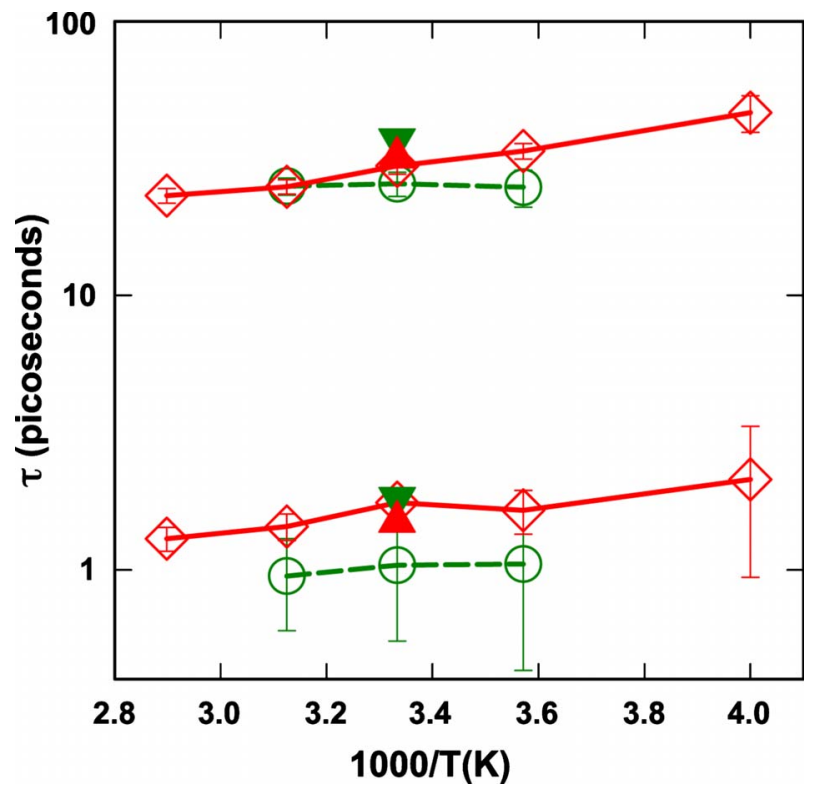

FIG. 37. (Color online) Characteristic residence times $(\tau)$ for fast (rotation) and intermediate (rotation-translation) diffusional components (open symbols) extracted from the QENS scattering from rutile and cassiterite nanoparticles surfaces (open symbols and dashed lines), compared with $\tau$ 's extracted form MD (filled symbols) over the same energy transfer range as the NIST DCS. Adapted from Mamontov et al., 2007. simple systems composed of very few atoms. However, new experimental probes are available to directly assess the level of rigor needed to capture the essential features of interfacial systems at the nanoscale. Furthermore, advances in computational capabilities can in turn be used to extract far more detail from scattering experiments than is apparent from the scattering signal alone, because the scattering signal can be simulated, and features of that signal can be assigned.

\section{FINDINGS AND RECOMMENDATIONS: LRI IN NS WORKSHOP}

From the workings of natural systems, by trial-anderror synthesis of new materials, by playing on the probabilistic aspects of the very small, by applying the mechanics of the very large, by mimicking the design of biological models, and by learning to work between the atomic microscopic and the materials macroscopic, we are creating a new science and engineering of "nanoscale" forces.

In this undertaking, there is healthy tension between the need to learn basic interaction fundamentals and the drive to realize practical powers of nanoscale forces. The particularities of solvation, fluctuation, structure, and electromagnetic fields acting at nanometer distances immediately compel us to learn new physics and chemistry as well as motivate us to design materials to new degrees of detail.

The concept of harnessing nanoscale forces evokes images of small low-energy control systems, colloids by design of controlled size, shape, and chemical functionality for catalysts; electronic devices, micro and nanomachines, and materials of "superdiamond" strength. Prototypical batteries and capacitors of high-energy density, solar arrays, photocatalysts, and high efficiency fuel cells are already being synthesized and tested. Most tantalizing is the potential mimicry of biological systems for energy conversion, efficient direct imaging, selfreproduction, and self-organization.

Systematic progress and leaps of invention both require knowledge of fundamental interactions beyond what we now possess. Intelligent recognition of this gap in determining research support can remove heavy impediments to practical invention. The greatest need in building a systematic science of nanometer-scale materials is to achieve reliable understanding and manipulation of their organizing forces.

Finally, because a line of inquiry can stop at a local minimum, it is beneficial to have external guidance and new perspectives to find global answers. For example, the discovery of the Casimir force was reportedly prompted by a simple remark by Niels Bohr during a conversation with Hendrik Casimir. Gathering together scientists of different fields facilitates these conversations. Fundamental insights created in this way can lead to transformative opportunities. Having prepared this review from such a gathering, we believe our work would not be complete without giving our recommendations for new lines of inquiry. 


\section{A. Recent scientific advances in LRI in NS}

Perhaps the most pleasant result from the reports on basic science had to do with electrodynamic (London dispersion, van der Waals, Hamaker, Casimir, and Lifshitz) forces. There has been rapid progress in formulations of geometries relevant to nanoscale systems. There is encouraging work on quantum mechanical computation of the material polarizabilities that animate these forces. There is also good progress in techniques to collect spectra needed for quantitative formulations.

Paradoxically, on the nanometer scale, the classic Coulomb and double-layer electrostatic forces that are widely encountered in most assembly processes are dauntingly intractable, this despite the statistical mechanical analyses that are being carried out on various idealized systems. Long range electric fields were, until the Debye-Huckel theory, the bane of early studies of dilute salt solutions. The correspondingly strong electric fields near ions still pose qualitative problems. Their polarizing forces are so strong as to reorganize water solvent so that continuum models break down. Ionic fields are so powerful as to approach those known to break down dielectrics. The chemical details of the ion orbitals enforce strict geometric conditions on surrounding waters. Even the polarizability of different ions creates charge-fluctuation forces as well as additional dielectric forces that are too often neglected. For these reasons, energies of individual ion solvation as well as ion interaction and ion approach to interfaces all occur under the dominance of high electric fields.

In the area of polar $(A B)$ and $\mathrm{H}$-bonded systems there has been good progress on specific problems but still little understanding of the powerful "hydration" forces that dominate interactions at approximately nanometer separations or even of the solvation of ions. As with electrostatics, there is an intrinsic connection between field-based and structural forces; and again as with electrostatics, there are few rigorously formulated and computed cases.

Over all these considerations is the obvious fact that real materials are organized by combinations of LRIs. They vary differently with solution conditions. The balance between them varies even more. The different degree of rigor and accuracy with which they can be computed frustrated attempts to achieve reliable combination and balance.

\section{B. Challenges and needs in LRI in NS}

Given the examples presented, it is at first difficult to see the defects and deficiencies impeding vigorous progress. The first point is the unevenness of that progress and the need to locate areas requiring new ideas and practices not currently the focus of active effort.

There is a striking disparity in the level of accuracy with which we can speak of the different kinds of interactions reviewed here. The general impression is that all kinds of interactions must be simultaneously connected.
When it comes to combining component forces in order to work with real systems, the chain of reasoning is as strong as its weakest link. That is to say, interactions are treated with such different degrees of approximation that their combination limits the strength of the enterprise to its most poorly understood component. We need reliable intermolecular force fields for computation. We need sufficient understanding to model many-body interactions. Particularly when working in the "nano" range, we must learn how to formulate effective interactions for various length scales.

Electrostatic and solvation interactions involve polarization, hydration, dielectric saturation, and structural components that carry us far beyond the continuumdielectric models that are still most popular. Reversing the scene of a half-century ago, it is the far more sophisticated theory of electrodynamic forces (van der Waals, Lifshitz, and Casimir) that earns reliability. Electrostatic and hydrogen-bonding polar interactions still frustrate quantitative evaluation. There are not enough test systems where measured forces are examined in sufficient detail that it is possible to test and validate quantitative theories and experimental procedures. Even within the world of computation and simulation there are not enough criteria procedures where results of the same problem can be compared so as to test different algorithms. There are even impediments in language: physicists think in terms of electrostatics and electrodynamics that sometimes do not easily relate to the chemist's structural approach.

The need for a merger of approaches is being systematically met on many fronts. Casimir forces are being formulated for arbitrary geometries. Simple analytical approximations are emerging that establish limits on behavior for different governing physics. There is an emerging, if overdue, recognition that it is necessary to join molecular dynamics with more accurate dispersion forces, generalized density functional theory, and quantum chemistry. More rigorous theory and computational algorithms, development of scattering, spectroscopic, and local probes of structure, forces, and dynamics at relevant time, space, and energy scales will combine to create practical theoretical tools. In this way one can expect new theoretical principles and practical implementations for real systems. Pushed to a natural limit, this effort will provide in-depth understanding of selfassembly for device manufacture.

How to proceed in this merger while keeping close to real systems? Many kinds of phenomena can prove instructive: collective dynamics, colloidal crystal selfassembly, and effective properties of zeolites and porous solids; properties of inhomogeneous liquids; processes driven by the defect interactions; ordered and amorphous interfaces of finite thickness; dimensionality and charge effects; charged defects and electrostatic fields on local phase stability and microscopic mechanisms of phase transitions and front motion; phase transitions in confined geometries-surface films and liquids; and concentrated solutions. 
From these we may expect new theoretical principles and implementations for real systems. The possibility exists to construct colloids by design-scalable synthetic methods that control size, shape, and chemical functionality of colloidal building blocks; the development of predictive tools for phase behavior, structure, and assembly for diverse motifs; the capability to control twoand three-dimensional electric and magnetic fields at nanoscale; and so on.

In this way, we expect to be able to merge not only physics, chemistry, and materials science and engineering approaches but also computational, experimental, and chemical methods for realistic interpretation and prediction.

\section{Transformative opportunities from LRI in NS}

The magic of the nanoscale (mixing the macromolecular with the macroscopic) creates a learning path to create new materials organized by long range forces. Even with the current rough ideas of colloid organizing forces, nanometer-size colloids or "tailored building blocks" are being synthesized with reliable uniformity so as to be suspended or manipulated to create instructive assemblies. But the range of such materials is still limited; theory and computation do not yet serve to describe or to design desired assemblies; and synthetic pathways are not yet ready for large supply.

Given needed support, the first two impediments will likely yield to current effort. The third, synthesis, needs bolder study but is not beyond achievement given the rapid progress in the theory and measurement of colloidal forces. Design of materials with temperature sensitive vdW-Ld forces and ion-conducting membranes show similar promise. Among the many tantalizing applications of carbon nanotubes as substrates for assembly and for elements in construction is the qualitative change to be expected from minor changes in carboncarbon networks. Given the dozens of windings in carbon atoms along the cylindrical shell, it becomes clear that some CNTs are simply dielectrics while others are conductors and still others are semiconductors whose electrical and charge-fluctuation properties can be switched by small changes in temperature. Obstacles to progress are the difficulties in synthesis of precisely defined CNTs as well as means to manipulate and watch them. None of these presents a major obstacle in principle, but practical procedures are nowhere within sight.

Batteries and high storage capacitors are perennial favorites on the must-make list for energy transforming materials. New materials such as the microbatteries from assembled $A B$ colloids and capacitors from highly polar nonreactive materials might begin to satisfy desiderata such as high-energy density, nontoxicity, durability, and inexpensive synthesis.

Manipulation of material polarizabilities creates the possibility of switchable friction between bodies whose polarizabilities can be changed by applied fields. The existence of such repulsion between surfaces has recently been reported. There is good reason to expect rapid progress in materials design.

\section{CONCLUSIONS}

Evidence gathered here reveals not only abundant creativity in the design of devices but also inspiring research on physical forces governing organization on the nanometer scale. Careful consideration of this research also shows an unevenness in our grasp of the basic organizing forces. Perhaps the greatest surprise is the inadequacy of theories of polar and electrostatic interactions compared with the present-day sophistication in formulating and computing charge-fluctuation forces. We cannot avoid these areas of ignorance. Electrostatics and polar interactions need conceptual advances. There is still no good algorithm to handle the strong electric fields near ions nor any language to include the powerful solvation forces surrounding even the simplest molecules.

Realizing that few systems operate by only one kind of interaction, we are faced with the paradox that the most sophisticated and accurate theory about one kind of force is vitiated when combined with less reliably formulated interactions. We were unanimous in our ardent plea that attention and significant research support be devoted to fundamental science. With better force measurement, theoretical formulation, and potentials for computer simulation, design of materials will accelerate and likely move faster than has been possible with trialand-error approaches.

A second fundamental need is education. There can be a healthy change in emphasis on learning to learn: better modes of teaching about forces as the need to learn them is recognized; better preparation in the basics of several sciences so as to remove the daunting fear of new learning. The possibilities can be realized in many ways, through supplementary coursework, improved computer facilities, and specialized texts that are written at a friendly level.

Then, third and greatest, there is the heavy work of designing and making materials, testing them, and creating synthetic pathways to provide needed supply. While theory and computation still fall short, the creation of materials is simultaneously a source of testing design ideas and of providing samples with systematically varied properties for systematic construction. It would qualitatively improve this iteration if material synthesis were made more accessible. With the magnificent facilities now being developed in national centers, including Department of Energy nanoscience centers, people will have new possibilities for design and application of design ideas. Training and linking programs that facilitate use of existing facilities is an economically practical strategy.

\section{ACKNOWLEDGMENTS}

We thank Harriet Kung, Director of Science, Office of Basic Energy Sciences (OBES) of the U.S. Department 
of Energy (DOE), and Frances Hellman, chairperson of the Council on Materials Science and Engineering of the Division of Materials Science and Engineering, in the OBES of the U.S. DOE and Arvind Kini of the U.S. DOE, OBES, Division of Materials Science and Engineering for sponsoring the workshop; Christie Ashton and Sophia Kitts for organizational assistance; and Barbara Brown French and Valerie Parsegian for assistance editing the manuscript. J.L. acknowledges support from an NSF CAREER award (Grant No. DMR 0448879, for studying SAFs), an AFOSR Young Investigator award (Grant No. FA9550-07-1-0125, for studying sintering and IGFs in W), and a DOE-BES grant (Grant No. DEFG02-08ER46511, for studying GB transitions in $\mathrm{Si}$ ). O.A.v.L. acknowledges support from SNL Truman Program LDRD under Project No. 120209. Sandia is a multiprogram laboratory operated by Sandia Corporation, a Lockheed Martin Co., for the United States Department of Energy's National Nuclear Security Administration under Contract No. DE-AC04-94AL85000. R.P. acknowledges support from the European Commission under Contract No. NMP3-CT-2005-013862 (INCEMS).

\section{REFERENCES}

Adão, M., B. J. V. Saramago, and A. C. Fernandes, 1999, J. Colloid Interface Sci. 217, 94.

Adjemian, K. T., R. Dominey, L. Krishnan, H. Ota, P. Majsztrik, T. Zhang, J. Mann, B. Kirby, L. Gatto, M. Velo-Simpson, J. Leahy, S. Srinivasan, J. B. Benziger, and A. B. Bocarsly, 2006, Chem. Mater. 18, 2238.

Agra, R., F. van Wijland, and E. Trizac, 2004, Phys. Rev. Lett. 93, 018304.

Ahuja, R., J. M. Osorio-Guillen, J. S. de Almeida, B. Holm, W. Y. Ching, and B. Johansson, 2004, J. Phys.: Condens. Matter 16, 2891.

Alberti, G., and M. Casciola, 2003, Annu. Rev. Mater. Res. 33, 129.

Alberti, G., M. Casciola, M. Pica, T. Tarpanelli, and M. Sganappa, 2005, Fuel Cells 5, 366.

Albrecht, T. R., P. Grütter, D. Horne, and D. Rugar, 1991, J. Appl. Phys. 69, 668.

Andelman, D., 1995, in Structure and Dynamics of Membrane, edited by R. Lipowsky and E. Sackmann (Elsevier, Amsterdam), Vol. 1B, p. 603.

Andersson, Y., D. C. Langreth, and B. I. Lundqvist, 1996, Phys. Rev. Lett. 76, 102.

Apetrei, A., I. Mirebeau, I. Goncharenko, D. Andreica, and P. Bonville, 2007, J. Phys.: Condens. Matter 19, 145214.

Appenzeller, J., J. Knoch, V. Derycke, R. Martel, S. Wind, and P. Avouris, 2002, Phys. Rev. Lett. 89, 126801.

Arnold, M. S., A. A. Green, J. F. Hulvat, S. I. Stupp, and M. C. Hersam, 2006, Nat. Nanotechnol. 1, 60.

Aryasetiawan, F., T. Miyake, and K. Terakura, 2002, Phys. Rev. Lett. 88, 166401.

Asthagiri, D., L. R. Pratt, and J. Kress, 2005, Proc. Natl. Acad. Sci. U.S.A. 102, 6704.

Asthagiri, D., L. R. Pratt, and M. Paulaitis, 2006, J. Chem. Phys. 125, 024701.

Ayton, G. S., W. G. Noid, and G. A. Voth, 2007, Curr. Opin. Struct. Biol. 17, 192.
Bachilo, S. M., M. S. Strano, C. Kittrell, R. H. Hauge, R. E. Smalle, and R. B. Weisman, 2002, Science 298, 2361.

Balazs, A., T. Emrick, and T. P. Russell, 2006, Science 314, 1107.

Balian, R., and B. Duplantier, 1978, Ann. Phys. (Paris) 112, 165.

Ballenegger, V., R. Blaak, and J.-P. Hansen, 2006, in Computer Simulations in Condensed Matter Systems: From Materials to Chemical Biology, edited by M. Ferrario, G. Ciccotti, and K. Binder, Lecture Notes in Physics Vol. 704 (Springer-Verlag, Berlin), p. 45.

Ballenegger, V., and J.-P. Hansen, 2005, J. Chem. Phys. 122, 114711.

Bandura, A. V., and J. D. Kubicki, 2003, J. Phys. Chem. B 107, 11072.

Barash, Y. Y., and O. I. Notysh, 1988, Sov. Phys. Solid State 30, 1580.

Bates, F. S., and G. H. Fredrickson, 1999, Phys. Today 52 (2), 32.

Baughman, R. H., A. A. Zakhidov, and W. A. de Heer, 2002, Science 297, 787.

Becke, A. D., and E. R. Johnson, 2005, J. Chem. Phys. 123, 154101.

Bee, M., 1988, Quasielastic Neutron Scattering: Principles and Applications in Solid State Chemistry, Biology and Materials Science (Hilger, Bristol).

Bellissent-Funel, M.-C., K. F. Bradley, S. H. Chen, J. Lal, and J. Teixeira, 1993, Physica A 201, 277.

Bellissent-Funel, M.-C., S. H. Chen, and J. M. Zanotti, 1995, Phys. Rev. E 51, 4558.

Benatov, L., and J. S. Wettlaufer, 2004, Phys. Rev. E 70, 061606.

Ben-Yaakov, D., D. Andelman, D. Harries, and R. Podgornik, 2009, J. Phys. Chem. B 113, 6001.

Berendsen, H. J. C., J. R. Grigera, and T. P. Straastma, 1987, J. Phys. Chem. 91, 6269.

Bertrand, E., et al., 2000, Phys. Rev. Lett. 85, 1282.

Bezrukov, S. M., I. Vodyanoy, and V. A. Parsegian, 1994, Nature (London) 370, 279.

Blake, N. P., M. K. Petersen, G. A. Voth, and H. Metiu, 2005, J. Phys. Chem. B 109, 24244.

Bonnet-Gonnet, C., S. Leikin, S. Chi, D. C. Rau, and V. A. Parsegian, 2001, J. Phys. Chem. B 105, 1877.

Bopp, P. A., A. A. Kornyshev, and G. Sutmann, 1998, J. Chem. Phys. 109, 1939.

Bordag, M., 2006, Phys. Rev. D 73, 125018.

Boroudjerdi, H., Y.-W. Kim, A. Naji, R. Netz, X. Schlagberger, and A. Serr, 2005, Phys. Rep. 416, 129.

Bos, R., H. C. van der Mel, and H. J. Busscher, 1999, FEMS Microbiol. Rev. 23, 179.

Boström, M., and B. E. Sernelius, 2000, Phys. Rev. B 61, 2204. Brochard, F., and P. G. de Gennes, 1991, Langmuir 7, 3216.

Brosseau, C., 2006, J. Tech. Phys. 39, 1277.

Brown, G. E., Jr., S. R. Sutton, and G. Calas, 2006, Elements 2, 9.

Brown, G. E., et al., 1999, Chem. Rev. (Washington, D.C.) 99, 77.

Brown-Hayes, M., D. A. R. Davit, F. D. Mazzitelli, W. J. Kim, and R. Onofrio, 2005, Phys. Rev. A 72, 052102.

Brydges, D. C., and P. A. Martin, 1999, J. Stat. Phys. 96, 1163. Büscher, R., and T. Emig, 2005, Phys. Rev. Lett. 94, 133901.

Bustamante, C., et al., 2003, Nature (London) 421, 423.

Butt, H. J., B. Cappella, and M. Kappl, 2005, Surf. Sci. Rep. 59, 
1.

Cahn, J. W., 1977, J. Chem. Phys. 66, 3667.

Cain, S. R., L. J. Matienzo, and F. Emmi, 1989, J. Phys. Chem. Solids 50, 87.

Cannon, R. M., and L. Esposito, 1999, Z. Metallkd. 90, 1002.

Cannon, R. M., et al., 2000, in Adsorption and Wetting Mechanisms at Ceramic Grain Boundaries Grain Boundary Engineering in Ceramics, edited by T. Sakuma and Y. Ikuhara (The American Ceramic Society, Westerville, OH), Vol. 118, p. 427.

Capasso, F., J. N. Munday, D. Iannuzzi, and H. B. Chan, 2007, IEEE J. Sel. Top. Quantum Electron. 13, 400.

Carriere, D., et al., 2007, Colloids Surf., A 303, 137.

Casimir, H. B. G., 1948, Proc. K. Ned. Akad. Wet. 51, 793.

Catalano, J. G., P. Fenter, and C. Park, 2007, Geochim. Cosmochim. Acta 71, 5313.

Cerny, J., and P. Hobza, 2005, Phys. Chem. Chem. Phys. 7, 1624.

Chakarova-Käck, S., E. Schröder, D. Langreth, and B. Lundqvist, 2008, Phys. Rev. Lett. 96, 146107.

Chan, H. B., V. A. Aksyuk, R. N. Kleinman, D. J. Bishop, and F. Capasso, 2001, Science 291, 1941.

Chattopadhyay, D., L. Galeska, and F. J. Papadimitrakopoulos, 2003, J. Am. Chem. Soc. 125, 3370.

Chaudhury, M. K., 1996, Mater. Sci. Eng. R. 16, 97.

Ching, W. Y., 1990, J. Am. Ceram. Soc. 73, 3135.

Chipot, C., and A. CPohorille, 2007, Eds., Free Energy Calculations: Theory and Applications in Chemistry and Biology (Springer, Berlin).

Cho, Y. K., R. Wartena, S. M. Tobias, and Y.-M. Chiang, 2007, Adv. Funct. Mater. 17, 379.

Cho, Y.-S., G.-R. Yi, J.-M. Lim, S.-H. Kim, V. N. Manoharan, D. J. Pine, and S.-M. Yang, 2005, J. Am. Chem. Soc. 127, 15968.

Churaev, N. A., B. Derjaguin, and V. Muller, 1987, Surface Forces (Springer, New York).

Clarke, D. R., 1987, J. Am. Ceram. Soc. 70, 15.

Cleveland, J. P., B. Anczykowski, A. E. Schmid, and V. B. Elings, 1998, Appl. Phys. Lett. 72, 2613.

Cole, D. R., K. W. Hervig, E. Mamontov, and J. Z. Larese, 2006, in Reviews in Mineralogy and Geochemistry 63, edited by H. R. Wenk (Mineralogical Society of America, Washington, D.C.), p. 313.

Collins, K. D., 2004, Methods 34, 300.

Colvin, V. L., M. C. Schlamp, and A. P. Alivisatos, 1994, Nature (London) 370, 354.

Cooper, V. R., T. Thonhauser, A. Puzder, E. Schröder, B. I. Lundqvist, and D. C. Langreth, 2008, J. Am. Chem. Soc. 130, 1304.

Correia, N. T., J. J. M. Ramos, B. J. V. Saramago, and J. C. G. Calado, 1997, J. Colloid Interface Sci. 189, 361.

Criscenti, L. J., and D. A. Sverjensky, 1999, Am. J. Sci. 299, 828.

Crupi, V., D. Majolino, P. Migliardo, and V. Venuti, 2002a, Physica A 304, 59.

Crupi, V., D. Majolino, P. Migliardo, and V. Venuti, 2002b, J. Phys. Chem. B 106, 10884.

Cygan, R. T., and J. D. Kubicki, 2001, Eds., Reviews in Mineralogy and Geochemistry (Mineralogical Society of America, Washington, D.C.), Vol. 42.

Dagastine, R. R., M. Bevan, L. R. White, and D. C. Prieve, 2004, J. Adhes. 80, 365.

Dantchev, D., F. Schlesener, and S. Dietrich, 2007, Phys. Rev.
E 76, 011121.

Dash, J. G., A. W. Rempel, and J. S. Wettlaufer, 2006, Rev. Mod. Phys. 78, 695.

Decca, R. S., D. Lopez, E. Fischbach, and D. E. Krause, 2003, Phys. Rev. Lett. 91, 050402.

de Heer, W. A., A. Châtelain, and D. Ugarte, 1995, Science 270, 1179.

Denk, W., and D. W. Pohl, 1991, Appl. Phys. Lett. 59, 2171.

Denzler, D. N., C. Hess, R. Dudek, S. Wagner, C. Frischkorn, M. Wolf, and G. Ertl, 2003, Chem. Phys. Lett. 376, 618.

Derjaguin, B. V., N. V. Churaev, and V. M. Muller, 1987, Surface Forces (Kluwer Academic, Dordrecht).

Derjaguin, B. V., N. A. Krotova, and V. P. Smilga, 1973, Adhesion of Solids (Nauka, Moscow) (in Russian).

Derjaguin, B. V., Y. I. Rabinovich, and N. V. Churaev, 1978, Nature (London) 272, 313.

Diebold, U., 2003, Surf. Sci. Rep. 48, 53.

Dietrich, S., 1988, in Phase Transitions and Critical Phenomena 12, edited by C. Domb and J. L. Lebowitz (Academic, London), p. 1.

Dietrich, S., and M. Napiórkowski, 1991, Phys. Rev. A 43, 1861.

Dillon, S. J., et al., 2007, Acta Mater. 55, 6208.

Dion, M., H. Rydberg, E. Schröder, D. C. Langreth, and B. I. Lundqvist, 2004, Phys. Rev. Lett. 92, 246401.

Dobson, J. F., and J. Wang, 1999, Phys. Rev. Lett. 82, 2123.

Dobson, J. F., A. White, and A. Rubio, 2006, Phys. Rev. Lett. 96, 073201.

Douillard, J. M., 1997, J. Colloid Interface Sci. 188, 511.

Drago, R. S., and B. Wayland, 1965, J. Am. Chem. Soc. 87, 3571.

Dzyaloshinskii, I. D., E. M. Lifshitz, and L. P. Pitaevskii, 1961, Usp. Fiz. Nauk 73, 381 [Sov. Phys. Usp. 4, 153 (1961)].

Edwards, E. W., D. Wang, and H. Mohwald, 2007, Macromol. Chem. Phys. 208, 439.

Egami, T., 2004, Z. Kristallogr. 219, 122.

Egami, T., 2007, J. Alloys Compd. 434-435, 110.

Eikerling, M., A. Kornyshev, and A. R. Kucernak, 2006, Phys. Today 59 (10), 38.

Eisenthal, K. B., 2006, Chem. Rev. (Washington, D.C.) 106, 1462.

Elbaum, M., and M. Schick, 1991, Phys. Rev. Lett. 66, 1713.

Elstner, M., P. Hobza, T. Frauenheim, S. Suhai, and E. Kaxiras, 2001, J. Chem. Phys. 114, 5149.

Emig, T., N. Graham, R. L. Jaffe, and M. Kardar, 2007, Phys. Rev. Lett. (to be published).

Emig, T., A. Hanke, R. Golestanian, and M. Kardar, 2003, Phys. Rev. A 67, 022114.

Emig, T., R. L. Jaffe, M. Kardar, and A. Scardicchio, 2006, Phys. Rev. Lett. 96, 080403.

Enderby, J. E., 1995, Chem. Soc. Rev. 24, 159.

Ennis, J., S. Marcelja, and R. Kjellander, 1996, Electrochim. Acta 41, 2115.

Evans, D. A., et al., 2003, Nucl. Instrum. Methods Phys. Res. B 199, 475.

Evans, D. F., and H. Wennerström, 1999, The Colloidal Domain: Where Physics, Chemistry, Biology and Technology Meet (Wiley, New York).

Evans, R., 1979, Adv. Phys. 28, 143.

Evans, R., 1992, in Fundamentals of Inhomogeneous Fluids, edited by D. Henderson (Dekker, New York), Chap. 3, p. 85. Faraone, A., L. Liu, C.-Y. Mou, P.-C. Shih, C. Brown, J. R. D. Copley, R. M. Dimeo, and S. H. Chen, 2003, Eur. Phys. J. E 
12, 59.

Faraone, A., L. Liu, C.-Y. Mou, P.-C. Shih, J. R. D. Copley, and S. H. Chen, 2003, J. Chem. Phys. 119, 3963.

Farrell, A. A., T. Fukuma, T. Uchihashi, E. R. Kay, G. Bottari, D. A. Leigh, H. Yamada, and S. P. Jarvis, 2005, Phys. Rev. B 72, 125430.

Fedkin, M. V., X. J. Zhou, J. D. Kubicki, A. V. Bandura, S. N. Lvov, M. L. Machesky, and D. J. Wesolowski, 2003, Langmuir 19, 3797.

Fenter, P., C. Park, K. L. Nagy, and N. C. Sturchio, 2007, Thin Solid Films 515, 5654.

Fenter, P. A., M. L. Rivers, N. C. Sturchio, and S. R. Sutton, 2002, Eds., Reviews in Mineralogy and Geochemistry (Mineralogical Society of America, Washington, D.C.), Vol. 49.

Fenter, P. A., and N. C. Sturchio, 2004, Prog. Surf. Sci. 77, 171. Feynman, R., 1960, Eng. Sci. 47, 22.

Ficker, T., A. Len, and P. Nemec, 2007, J. Tech. Phys. 40, 4055.

Finnis, M. W., 2003, Interatomic Forces in Condensed Matter (Oxford University Press, Oxford).

Fisher, M. E., and P.-G. de Gennes, 1978, C. R. Seances Acad. Sci., Ser. B 287, 207.

Fitts, J. P., M. L. Machesky, D. J. Wesolowski, X. Shang, J. D. Kubicki, G. W. Flynn, T. F. Heinz, and K. B. Eisenthal, 2005, Chem. Phys. Lett. 411, 399.

Foster, A., and W. Hofer, 2006, Scanning Probe Microscopy: Atomic Scale Engineering by Forces and Currents (Springer, New York).

Fowkes, F. M., 1953, J. Phys. Chem. 57, 98.

Fowkes, F. M., 1963, J. Phys. Chem. 67, 2538.

Fowkes, F. M., 1983, in Physicochemical Aspects of Polymeric Surfaces, edited by K. L. Mittal (Plenum, New York), Vol. 2, p. 583.

Frederix, P. L. T. M., M. R. Gullo, T. Akiyama, A. Tonin, N. F. de Rooij, U. Staufer, and A. Engel, 2005, Nanotechnology 16, 997.

French, R. H., 2000, J. Am. Ceram. Soc. 83, 2117.

French, R. H., K. I. Winey, M. K. Yang, and W. Qiu, 2007, Aust. J. Chem. 60, 251.

Fuchs, M., and X. Gonze, 2002, Phys. Rev. B 65, 235109.

Fukushi, K., and D. A. Sverjensky, 2007, Geochim. Cosmochim. Acta 71, 3717.

Fukuto, M., Y. F. Yano, and P. S. Pershan, 2005, Phys. Rev. Lett. 94, 135702.

Furche, F., and T. Van Voorhis, 2005, J. Chem. Phys. 122, 164106.

Gang, O., K. Alvine, M. Fukuto, P. Pershan, C. Black, and B. M. Ocko, 2005, Phys. Rev. Lett. 95, 217801.

Ganshin, A., S. Scheidemantel, R. Garcia, and M. H. W. Chan, 2006, Phys. Rev. Lett. 97, 075301.

Garcia, R., and M. H. W. Chan, 1999, Phys. Rev. Lett. 83, 1187. Garcia, R., and M. H. W. Chan, 2002, Phys. Rev. Lett. 88, 086101.

Gies, H., and K. Klingmuller, 2006, Phys. Rev. D 74, 045002.

Glotzer, S. C., and M. J. Solomon, 2007, Meteorit. Planet. Sci. 6, 557.

Glover, J. L., and C. T. Chantler, 2007, Meas. Sci. Technol. 18, 2916.

Goedecker, S., M. Teter, and J. Hutter, 1996, Phys. Rev. B 54, 1703.

Gomes, D., L. Buder, and S. P. Nunes, 2006, J. Polym. Sci., Part B: Polym. Phys. 44, 2278.

Goncharenko, I. N., 2005, J. Phys.: Condens. Matter 17, S947.

González-Mozuelos, P., and N. Bagatella-Flores, 2000, Physica
A 286, 56 .

Grimme, S., J. Antony, T. Schwabe, and C. Mück-Lichtenfeld, 2007, Org. Biomol. Chem. 5, 741.

Grosberg, A. Y., T. T. Nguyen, and B. I. Shklovskii, 2002, Rev. Mod. Phys. 74, 329.

Grossfield, A., P. Y. Ren, and J. Ponder, 2003, J. Am. Chem. Soc. 125, 15671.

Gruen, D., and S. Marcelja, 1983, J. Chem. Soc., Faraday Trans. 2 79, 211.

Gruner, G., 2006, Anal. Bioanal. Chem. 384, 322.

Grütter, P., Y. Liu, P. LeBlanc, and U. Dürig, 1997, Appl. Phys. Lett. 71, 279.

Gu, L. Q., O. Braha, S. Conlan, S. Cheley, and H. Bayley, 1999, Nature (London) 398, 686.

Guldbrand, L., B. Jönsson, H. Wennerström, and P. Linse, 1984, J. Chem. Phys. 80, 2221.

Guldbrand, L., L. G. Nilsson, and L. Nordenskiöld, 1986, J. Chem. Phys. 85, 6686.

Guo, J., S. Goasguen, M. Lundstrom, and S. Datta, 2002, Appl. Phys. Lett. 81, 1486.

Guo, J., M. Lundstrom, and S. Datta, 2002, Appl. Phys. Lett. 80, 3192.

Gupta, V. K., et al., 2007, Acta Mater. 55, 3131.

Gur, I., N. A. Fromer, M. L. Geier, and A. P. Alivisatos, 2005, Science 310, 462.

Gurnev, P. A., D. Harries, V. A. Parsegian, and S. M. Bezrukov, 2009, ChemPhysChem 10, 1445.

Gutmann, V., 1978, The Donor-Acceptor Approach to Molecular Interactions (Plenum, New York).

Hao, J. C., and T. Zemb, 2007, Curr. Opin. Colloid Interface Sci. 12, 129.

Harnau, L., and S. Dietrich, 2004, Phys. Rev. E 69, 051501. Harries, D., and V. A. Parsegian, 2004, Proteins 57, 311. Harries, D., and J. Rosgen 2008, Methods Cell Biol. 84, 679.

Harrison, W. L., M. A. Hickner, Y. S. Kim, and J. E. McGrath, 2005, Fuel Cells 5, 201.

Hayward, S. A., S. A. T. Redfern, H. J. Stone, M. G. Tucker, K. R. Whittle, and W. G. Marshall, 2005, Z. Kristallogr. 220, 735. Heinze, S., J. Tersoff, R. Martel, V. Derycke, J. Appenzeller, and Ph. Avouris, 2002, Phys. Rev. Lett. 89, 106801.

Heitler, W., and F. London, 1927, Z. Phys. 44, 455.

Hellmann, H., 1935, J. Chem. Phys. 3, 61.

Henning, A. K., T. Hochwitz, J. Slinkman, J. Never, S. Hoffman, P. Kaszuba, and C. Daghlian, 1995, J. Appl. Phys. 77, 1888.

Hertlein, C., L. Helden, A. Gambassi, S. Dietrich, and C. Bechinger, 2008, Nature (London) 451, 172.

Heßelmann, A., and G. Jansen, 2003, Phys. Chem. Chem. Phys. 5, 5010.

Hickner, M. A., H. Ghassemi, Y. S. Kim, B. R. Einsla, and J. E. McGrath, 2004, Chem. Rev. (Washington, D.C.) 104, 4587.

Hickner, M. A., and B. S. Pivovar, 2005, Fuel Cells 5, 213.

Hiemstra, T., P. Venema, and W. H. Van Riemsdijk, 1996, J. Colloid Interface Sci. 184, 680.

Ho, P. S., R. Haight, R. C. Silverman, B. D. White, and F. Faupel, 1991, in Fundamentals of Adhesion, edited by L. H. Lee (Plenum, New York), p. 383.

Hofer, W. A., A. S. Foster, and A. L. Shluger, 2003, Rev. Mod. Phys. 75, 1287.

Hoffmann, N., C. N. Likos, and J.-P. Hansen, 2004, Mol. Phys. $102,857$.

Hohenberg, P., and W. Kohn, 1964, Phys. Rev. 136, B864.

Holm, C., P. Kekicheff, and R. Podgornik, 2001, Electrostatic 
Effects in Soft Matter and Biophysics (Kluwer Academic, Dordecht).

Hong, L., A. Cacciuto, E. Luijten, and S. Granick, 2006, Arch. Hist. Exact Sci. 6, 2510.

Hribar, B., and V. Vlachy, 2000, Biophys. J. 78, 694.

Huang, Y., X. F. Duan, Y. Cui, L. J. Lauhon, K. H. Kim, and C. M. Lieber, 2001, Science 294, 1313.

Hudson, R. F., and G. Klopman, 1967, Tetrahedron Lett. 8, 1103.

Hult, E., H. Rydberg, B. I. Lundqvist, and D. C. Langreth, 1998, Phys. Rev. B 59, 4708.

Hunter, R. J., 1989, Foundations of Colloidal Science (Clarendon, Oxford), Vol. 1.

Hunter, R. J., 2001, Foundations of Colloidal Science, 2nd ed. (Oxford University Press, Oxford).

Hynninen, A.-P., and A. Z. Panagiotopoulos, 2007, Phys. Rev. Lett. 98, 198301.

Iftimie, R., P. Minary, and M. E. Tuckerman, 2005, Proc. Natl. Acad. Sci. U.S.A. 102, 6654.

Ikeda, A., T. Hamano, K. Hayashi, and J. Kikuchi, 2006, Org. Lett. 8, 1153.

Israelachvili, J. N., 2006, Intermolecular and Surface Forces (Academic, New York).

Israelachvili, J. N., and G. J. Adams, 1978, J. Chem. Soc., Faraday Trans. $1 \mathbf{7 4 ,} 975$.

Jancovici, B., 1982, J. Stat. Phys. 29, 263.

Jang, S. S., V. Molinero, T. Çağin, and W. A. Goddard III, 2004, J. Phys. Chem. B 108, 3149.

Javey, A., J. Guo, Q. Wang, M. Lundstrom, and H. J. Dai, 2003, Nature (London) 424, 654.

Jesse, S., S. V. Kalinin, R. Proksch, A. P. Baddorf, and B. J. Rodriguez, 2007, Nanotechnology 18, 435503.

Johnson, E. R., and A. D. Becke, 2006, J. Chem. Phys. 124, 174104.

Johnson, R. R., A. T. Johnson, and M. L. Klein, 2008, Nano Lett. 8, 69.

Johnston, K., and M. Finnis, 2002, J. Am. Ceram. Soc. 85, 2562. Jönsson, B., A. Nonat, C. Labbez, B. Cabane, and H. Wennerström, 2005, Langmuir 21, 9211.

Jung, J., P. Garcia-Gonzalez, J. F. Dobson, and R. W. Godby, 2004, Phys. Rev. B 70, 205107.

Jungwirth, P., and D. J. Tobias, 2006, Chem. Rev. (Washington, D.C.) 106, 1259.

Kaewsaiha, P., K. Matsumoto, and H. Matsuoka, 2007, Langmuir 23, 9162.

Kalinin, S., B. Rodriguez, S. Jesse, E. Karapetian, B. Mirman, E. Eliseev, and A. Morozovska, 2007, Annu. Rev. Mater. Res. 37, 189.

Kalinin, S. V., B. J. Rodriguez, S. Jesse, T. Thundat, and A. Gruverman, 2005, Appl. Phys. Lett. 87, 053901.

Kanduc, M., J. Dobnikar, and R. Podgornik, 2009, Soft Matter $\mathbf{5}, 868$.

Kanduc, M., A. Naji, Y. S. Jho, P. A. Pincus, and R. Podgornik, 2009, J. Phys.: Condens. Matter 21, 424103.

Kantorovich, L. N., and T. Trevethan, 2004, Phys. Rev. Lett. 93, 236102.

Kékicheff, P., S. Marcelja, T. J. Senden, and V. E. Shubin, 1993, J. Chem. Phys. 99, 6098.

Kenneth, O., and I. Klich, 2006, Phys. Rev. Lett. 97, 160401.

Khandpur, A. K., S. Forster, F. S. Bates, I. W. Hamley, A. J. Ryan, W. Bras, K. Almdal, and K. Mortensen, 1995, Macromolecules 28, 8796.

Kim, E., et al., 2007, J. Phys. Chem. B 111, 13873.
Kim, H.-J., Y.-G. Shul, and H. Han, 2006, J. Power Sources 158, 137.

Kimber, S. A. J., A. C. McLaughlin, and J. P. Attfield, 2006, Mater. Res. Bull. 41, 1001.

Kjellander, R., and B. Forsberg, 2005, J. Phys. A 38, 5405.

Kjellander, R., and S. Marcelja, 1984, Chem. Phys. Lett. 112, 49.

Kjellander, R., S. Marcelja, and J. P. Quirk, 1988, J. Colloid Interface Sci. 126, 194.

Kjellander, R., and R. Ramirez, 2005, J. Phys.: Condens. Matter 17, S3409.

Kleis, J., B. I. Lundqvist, D. C. Langreth, and E. Schröder, 2007, Phys. Rev. B 76, 100201(R).

Knecht, V., H. J. Risselada, A. E. Mark, and S. J. Marrink, 2008, J. Colloid Interface Sci. 318, 477.

Knowles, K. M., 2005, J. Ceram. Proc. Res. 6, 10.

Koch, W., and M. C. Holthausen, 2001, A Chemist's Guide to

Density Functional Theory (Wiley-VCH, New York).

Kohn, W., and L. J. Sham, 1965, Phys. Rev. 140, A1133.

Koretsky, C. M., D. A. Sverjensky, and N. Sahai, 1998, Am. J. Sci. 298, 349.

Kornyshev, A., D. J. Lee, S. Leikin, and A. Wynveen, 2007, Rev. Mod. Phys. 79, 943.

Krech, M., 1994, The Casimir Effect in Critical Systems (World Scientific, Singapore).

Krech, M., and S. Dietrich, 1991, Phys. Rev. Lett. 66, 345.

Krech, M., and S. Dietrich, 1992, Phys. Rev. A 46, 1886.

Kreitmeir, M., H. Bertagnolli, K. Toedheide, and A. K. Soper, 2007, Z. Phys. Chem. 221, 801.

Kreuer, K.-D., S. J. Paddison, E. Spohr, and M. Schuster, 2004, Chem. Rev. (Washington, D.C.) 104, 4637.

Kristyán, S., and P. Pulay, 1994, Chem. Phys. Lett. 229, 175.

Krupke, R., F. Hennrich, H. V. Lohneysen, and M. M. Kappes, 2003, Science 301, 344.

Kunz, W., P. Lo Nostro, and B. W. Ninham, 2004, Curr. Opin. Colloid Interface Sci. 9, 1.

Kurth, S., J. P. Perdew, and P. Blaha, 1999, Int. J. Quantum Chem. 75, 889 .

Labbez, C., A. Nonat, I. Pochard, and B. Jönsson, 2007, J. Colloid Interface Sci. 309, 303.

Lamoreaux, S. K., 1997, Phys. Rev. Lett. 78, 5.

Lamoreaux, S. K., 2005, Rep. Prog. Phys. 68, 201.

Larrimore, L., S. Nad, X. Zhou, H. Abruna, and P. L. McEuen, 2006, Arch. Hist. Exact Sci. 6, 1329.

Lee, L. H., 1998, J. Adhes. 67, 1.

Lee, N. S., et al., 2001, Diamond Relat. Mater. 10, 265.

Lee, S. S., K. L. Nagy, and P. Fenter, 2007, Geochim. Cosmochim. Acta 71, 5763.

Lee, S. W., and W. M. Sigmund, 2002, Colloids Surf., A 204, 43. Leikin, S., V. A. Parsegian, D. C. Rau, and R. P. Rand, 1993, Annu. Rev. Phys. Chem. 44, 369.

Leunissen, M. E., C. G. Christova, A.-P. Hynninen, C. P. Royall, A. I. Campbell, A. Imhof, M. Dijkstra, R. van Roij, and A. van Blaaderen, 2005, Nature (London) 437, 235.

Licoccia, S., and E. Traversa, 2006, J. Power Sources 159, 12. Lifshitz, E. M., 1955, Zh. Eksp. Teor. Fiz. 29, 894.

Lifshitz, E. M., 1956, Sov. Phys. JETP 2, 73.

Lin, I.-C., M. D. Coutinho-Neto, C. Felsenheimer, O. A. von Lilienfeld, I. Tavernelli, and U. Rothlisberger, 2007, Phys. Rev. B 75, 205131.

Lin, I.-C., A. P. Seitsonen, M. D. Coutinho-Neto, I. Tavernelli, and U. Rothlisberger, 2009, J. Phys. Chem. B 113, 1127.

Lin, I.-C., O. A. von Lilienfeld, M. Coutinho-Neto, I. Tavern- 
elli, and U. Rothlisberger, 2007, J. Chem. Phys. 111, 14346.

Linse, P., 2005, in Advanced Computer Simulation Approaches in Soft Matter Sciences II, edited by C. Holm and K. Kremer (Springer, New York), Vol. 185, p. 111

Liu, J., and E. Luijten, 2005, Phys. Rev. E 72, 061401.

Liu, J., H. J. Schope, and T. Palberg, 2002, J. Chem. Phys. 116, 5901.

Liu, L., A. Faraone, C.-Y. Mou, P.-C. Shih, and S. H. Chen, 2004, J. Phys.: Condens. Matter 16, S5403.

Lobaskin, V., A. Lyubartsev, and P. Linse, 2001, Phys. Rev. E 63, 020401.

Lozada-Cassou, M., R. Saavedra-Barrera, and D. Henderson, 1982, J. Chem. Phys. 77, 5150.

Luo, J., 2007, Crit. Rev. Solid State Mater. Sci. 32, 67.

Luo, J., 2008, Curr. Opin. Solid State Mater. Sci. 12, 81.

Luo, J., and Y.-M. Chiang, 2008, Annu. Rev. Mater. Res. 38, 227.

Luo, J., Y.-M. Chiang, and R. Cannon, 2005, Langmuir 21, 7358.

Luo, J., V. K. Gupta, D. H. Yoon, and H. M. Meyer, 2005, Appl. Phys. Lett. 87, 231902.

Luo, J., and X. Shi, 2008, Appl. Phys. Lett. 92, 101901.

Luo, J., et al., 1999, J. Am. Ceram. Soc. 82, 1922.

Luo, J., et al., 2006, Mater. Sci. Eng., A 422, 19.

Lustig, S. R., A. Jagota, C. Khripin, and M. Zheng, 2005, J. Phys. Chem. B 109, 2559.

Lützenkirchen, J., 2006, Ed., Surface Complexation Modelling (Elsevier, Amsterdam).

Lutzenkirchen-Hecht, D., P. Keil, and R. Frahm, 2007, Surf. Sci. 601, 4232.

Lynch, B. P., A. M. Hilton, and G. J. Simpson, 2006, Biophys. J. 91, 2678

Lyubartsev, A. P., and A. Laaksonen, 1995, Phys. Rev. E 52, 3730.

Lyubartsev, A. P., and A. Laaksonen, 2004, in Novel Methods in Soft Matter Simulations, edited by M. Karttunen, I. Vattulainen, and A. Lukkarinen, Lecture Notes in Physics Vol. 640 (Springer-Verlag, Berlin), p. 219.

Machesky, M. L., D. J. Wesolowski, D. A. Palmer, and M. K. Ridley, 2001, J. Colloid Interface Sci. 239, 314.

Maciolek, A., and S. Dietrich, 2006, Europhys. Lett. 74, 22.

Maciołek, A., A. Gambassi, and S. Dietrich, 2007, Phys. Rev. E 76, 031124.

Mahanty, J., and B. W. Ninham, 1976, Dispersion Forces (Academic, London).

Mamontov, E., 2004, J. Chem. Phys. 121, 9087.

Mamontov, E., 2005a, J. Chem. Phys. 123, 024706.

Mamontov, E., 2005b, J. Chem. Phys. 123, 171101.

Mamontov, E., L. Vicek, D. J. Wesolowski, P. T. Cummings, W. Wang, L. M. Anovitz, J. Rosenquist, C. M. Brown, and V. Garcia-Sakai, 2007, J. Phys. Chem. C 111, 4328.

Mamontov, E., D. J. Wesolowski, L. Vlcek, P. T. Cummings, J. Rosenqvist, W. Wang, and D. R. Cole, 2008, J. Phys. Chem. C 112, 12334.

Manna, L., E. C. Scher, and A. P. Alivisatos, 2000, J. Am. Chem. Soc. 122, 12700.

Manohar, S., T. Tang, and A. Jagota, 2007, J. Phys. Chem. C 111, 17835.

Manoharan, V. N., M. T. Elsesser, and D. J. Pine, 2003, Science 301, 483.

Mansour, F., R. M. Dimeo, and H. Peemoeller, 2002, Phys. Rev. E 66, 041307.

Marini, A., P. Garcia-Gonzalez, and A. Rubio, 2006, Phys. Rev.
Lett. 96, 136404.

Marques, M. A. L., A. Castro, G. Malloci, G. Mulas, and S. Botti, 2007, J. Chem. Phys. 127, 014107.

Martinez, C. J., J. Liu, S. K. Rhodes, E. Luijten, E. R. Weeks, and J. A. Lewis, 2005, Langmuir 21, 9978.

Mate, C. M., G. M. McClelland, R. Erlandsson, and S. Chiang, 1987, Phys. Rev. Lett. 59, 1942.

Matthies, S., J. Pehl, H. R. Wenk, L. Lutterotti, and S. C. Vogel, 2005, J. Appl. Crystallogr. 38, 462.

Mauritz, K. A., and R. B. Moore, 2004, Chem. Rev. (Washington, D.C.) 104, 4535.

Mavon, A., D. Redoules, P. Humbert, P. Agache, and Y. Gall, 1998, Colloids Surf., B 10, 243.

McLean, R. S., X. Huang, C. Khripin, A. Jagota, and M. Zheng, 2006, Nano Lett. 6, 55.

Meijer, E. J., and M. Sprik, 1996, J. Chem. Phys. 105, 8684.

Mishchenko, M. I., et al. 2004, J. Quant. Spectrosc. Radiat. Transf. 88, 357..

Misquitta, A. J., B. Jeziorski, and K. Szalewicz, 2003, Phys.

Rev. Lett. 91, 033201.

Miyake, T., F. Aryasetiawan, T. Kotani, M. van Schilfgaarde, M. Usuda, and K. Terakura, 2002, Phys. Rev. B 66, 245103.

Miyatani, T., M. Horii, A. Rosa, M. Fujihira, and O. Marti, 1997, Appl. Phys. Lett. 71, 2632.

Mohideen, U., and A. Roy, 1998, Phys. Rev. Lett. 81, 4549.

Mohraz, A., E. R. Weeks, and J. A. Lewis, 2008, Phys. Rev. E 77, 060403.

Moosavi, A., M. Rauscher, and S. Dietrich, 2006, Phys. Rev. Lett. 97, 236101.

Moosavi, A., M. Rauscher, and S. Dietrich, 2008a, Langmuir 24, 734 .

Moosavi, A., M. Rauscher, and S. Dietrich, 2008b, J. Chem. Phys. 129, 044706.

Moreira, A. G., and R. R. Netz, 2002, Eur. Phys. J. E 8, 33.

Muenter, A. H., J. L. DeZwaan, and G. M. Nathanson, 2007, J. Phys. Chem. C 111, 15043.

Munday, J. N., and F. Capasso, 2007, Phys. Rev. A 75, 060102(R).

Munday, J. N., F. Capasso, and V. A. Parsegian, 2009, Nature (London) 457, 170.

Naji, A., S. Jungblut, A. G. Moreira, and R. R. Netz, 2005, Physica A 352, 131.

Nave, S., C. Mandin, L. Martinet, L. Berthon, F. Testard, C. Madic, and T. Zemb, 2004, Phys. Chem. Chem. Phys. 6, 799. Neilson, G. W., P. E. Mason, S. Ramos, and D. Sullivan, 2001, Philos. Trans. R. Soc. London, Ser. A 359, 1575.

Netz, R. R., 2001, Eur. Phys. J. E 5, 557.

Newton, R. G., 1966, Scattering Theory of Waves and Particles (McGraw-Hill, New York).

Ninham, B. W., and V. Yaminsky, 1997, Langmuir 13, 2097.

Nonnenmacher, M., M. P. O'Boyle, and H. K. Wickramasinghe, 1991, Appl. Phys. Lett. 58, 2921.

Noy, A., C. D. Frisbie, L. F. Rozsnyai, M. S. Wrighton, and C. M. Lieber, 1995, J. Am. Chem. Soc. 117, 7943.

O'Connell, M. J., P. Boul, L. M. Ericson, C. Huffman, Y. Wang, E. Haroz, C. Kuper, J. Tour, K. K. Ausman, and R. E. Smalley, 2001, Chem. Phys. Lett. 342, 256.

Ogasawara, H., B. Brena, D. Nordlund, M. Nyberg, A. Pelmenschikov, L. Pettersson, and A. Nilsson, 2002, Phys. Rev. Lett. 89, 276102.

Olson, W. K., A. A. Gorin, X.-J. Lu, L. M. Hock, and V. B. Zhurkin, 1998, Proc. Natl. Acad. Sci. U.S.A. 95, 11163.

Olson, W. K., et al., 2001, J. Mol. Biol. 313, 229. 
Ong, Y.-L., A. Razatos, G. Georgiou, and M. M. Sharma, 1999, Langmuir 15, 2719.

Oosawa, F., 1971, Polyelectrolytes (Dekker, New York), p. 123. Ortmann, F., F. Bechstedt, and W. G. Schmidt, 2006, Phys. Rev. B 73, 205101.

Ortmann, F., W. G. Schmidt, and F. Bechstedt, 2005, Phys. Rev. Lett. 95, 186101.

Outhwaite, C. W., and L. B. Bhuiyan, 1983, J. Chem. Soc., Faraday Trans. 2 79, 707.

Paliwal, A., et al., 2006, J. Chem. Phys. 124, 224502.

Parac, M., M. Etinski, M. Peric, and S. Grimme, 2005, J. Chem. Theory Comput. 1, 1110.

Park, C., and P. A. Fenter, 2007, J. Appl. Crystallogr. 40, 290.

Park, H., J. Jung, D. K. Min, S. Kim, S. Hong, and H. Shin, 2004, Appl. Phys. Lett. 84, 1734.

Parr, R. C., and R. G. Pearson, 1983, J. Am. Chem. Soc. 105, 7512.

Parr, R. G., and W. Yang, 1989, Density-Functional Theory of Atoms and Molecules (Oxford University Press, New York).

Parsegian, V. A., 2005, Van der Waals Forces (Cambridge University Press, Cambridge).

Parsegian, V. A., R. P. Rand, N. L. Fuller, and D. C. Rau, 1986, Methods Enzymol. 127, 400.

Parsegian, V. A., and G. H. Weiss, 1972, J. Adhes. 3, 259.

Peppin, S. S. L., J. A. W. Elliott, and M. G. Worster, 2006, J. Fluid Mech. 554, 147.

Peppin, S. S. L., J. S. Wettlaufer, and M. G. Worster, 2008, Phys. Rev. Lett. 100, 238301.

Peppin, S. S. L., M. G. Worster, and J. S. Wettlaufer, 2007, Proc. R. Soc. London, Ser. A 463, 723.

Pérez-Jordá, J. M., A. D. Becke, and E. San-Fabian, 1994, J. Chem. Phys. 100, 6520.

Pitarke, J. M., and J. P. Perdew, 2003, Phys. Rev. B 67, 045101.

Podgornik, R., R. H. French, and V. A. Parsegian, 2006, J. Chem. Phys. 124, 044709.

Podgornik, R., D. Harries, J. DeRouchey, H. H. Strey, and V. A. Parsegian, 2008, in Interactions in Macromolecular Complexes Used as Nonviral Vectors for Gene Delivery, in Gene Therapy: Therapeutic Mechanisms and Strategies, 3rd ed., edited by N. Smyth-Templeton (Dekker, New York).

Podgornik, R., H. H. Strey, and V. A. Parsegian, 1998, Curr. Opin. Colloid Interface Sci. 3, 534.

Poon, W. C. K., and D. Andelman, 2006, Soft Condensed Matter Physics in Molecular and Cell Biology (Taylor \& Francis, New York).

Poynor, A., L. Hong, I. K. Robinson, S. Granick, Z. Zhang, and P. A. Fenter, 2006, Phys. Rev. Lett. 97, 266101.

Prasad, V., D. Semwogerere, and E. R. Weeks, 2007, J. Phys.: Condens. Matter 19, 113102.

Předota, M., A. V. Bandura, P. T. Cummings, J. D. Kubicki, D. J. Wesolowski, A. A. Chialvo, and M. L. Machesky, 2004, J. Phys. Chem. B 108, 12049.

Předota, M., and L. Vlcek, 2007, J. Phys. Chem. B 111, 1245.

Předota, M., Z. Zhang, P. Fenter, D. J. Wesolowski, and P. T. Cummings, 2004, J. Phys. Chem. B 108, 12061.

Proksch, R., K. Babcock, and J. Cleveland, 1999, Appl. Phys. Lett. 74, 419.

Qi, P., V. Ophir, M. Grecu, A. Javey, Q. Wang, and H. Dai, 2003, Arch. Hist. Exact Sci. 3, 347.

Qian, H., and J. Luo, 2007, Appl. Phys. Lett. 91, 061909.

Qian, H. J., J. Luo, and Y.-M. Chiang, 2008, Acta Mater. 56, 862.

Qin, L., S. Park, L. Huang, and C. A. Mirkin, 2005, Science
309, 113.

Raab, A., W. Han, D. Badt, S. J. Smith-Gill, S. M. Lindsay, H. Schindler, and P. Hinterdorfer, 1999, Nat. Biotechnol. 17, 901. Rahi, J. S., A. Rodriguez, S. G. Johnson, T. Emig, R. L. Jaffe, and M. Kardar, 2008, Phys. Rev. A 77, 030101(R).

Rahman, A., and F. Stillinger, 1971, J. Chem. Phys. 55, 3336. Rajter, R., and R. H. French, 2010, Int. J. Mater. Res. 101, 27. Rajter, R. F., and R. H. French, 2008, J. Phys.: Conf. Ser. 94, 012001.

Rajter, R. F., R. H. French, R. Podgornik, W. Y. Ching, and A. Parsegian, 2008, J. Appl. Phys. 104, 053513.

Rajter, R. F., R. Podgornik, V. A. Parsegian, R. H. French, and W. Y. Ching, 2007, Phys. Rev. B 76, 045417.

Ramirez, R., and R. Kjellander, 2006, J. Chem. Phys. 125, 144110.

Rau, D. C., and V. A. Parsegian, 1992, Biophys. J. 61, 246.

Raviv, U., and J. Klein, 2002, Science 297, 1540.

Rempel, A. W., J. S. Wettlaufer, and E. D. Waddington, 2002, J. Geophys. Res. 107, 2330.

Rempel, A. W., J. S. Wettlaufer, and M. Worster, 2001, Phys. Rev. Lett. 87, 088501.

Rempel, A. W., and M. G. Worster, 2001, J. Cryst. Growth 223, 420.

Reščič, J., and P. Linse, 2001, J. Chem. Phys. 114, 10131.

Rheinstädter, M. C., T. Seydel, B. Farago, and T. Salditt, 2006, J. Neutron Res. 14, 257.

Ricoul, F., M. Dubois, L. Belloni, and T. Zemb, 1998, Langmuir 14, 2645.

Rief, M., F. Oesterhelt, B. Heymann, and H. E. Gaub, 1997, Science 275, 1295.

Ritort, F., 2006, J. Phys.: Condens. Matter 18, R531.

Rodriguez, A., M. Ibanescu, D. Iannuzzi, F. Capasso, J. D. Joannopoulos, and S. G. Johnson, 2007, Phys. Rev. Lett. 99, 080401.

Rodriguez, A., M. Ibanescu, D. Iannuzzi, J. D. Joannopoulos, and S. G. Johnson, 2007, Phys. Rev. A 76, 032106.

Rodriguez, B. J., C. Callahan, S. V. Kalinin, and R. Proksch, 2007, Nanotechnology 18, 475504.

Rodriguez, B. J., S. Jesse, A. P. Baddorf, and S. V. Kalinin, 2006, Phys. Rev. Lett. 96, 237602.

Rodriguez, B. J., S. Jesse, A. P. Baddorf, S. H. Kim, and S. V. Kalinin, 2007, Phys. Rev. Lett. 98, 247603.

Rosenblatt, S., Y. Yaish, J. Park, J. Gore, V. Sazonova, and P. L. McEuen, 2002, Arch. Hist. Exact Sci. 2, 869.

Rosner, B. T., and D. W. van der Weide, 2000, Rev. Sci. Instrum. 73, 2505.

Roth, R., R. Evans, and S. Dietrich, 2000, Phys. Rev. E 62, 5360.

Rother, G., Y. B. Melnichenko, D. R. Cole, H. Frielinghaus, and G. D. Wignall, 2007, J. Phys. Chem. C 111, 15736.

Rowan, D. G., J.-P. Hansen, and E. Trizac, 2000, Mol. Phys. 98, 1369.

Roy, A., C. Y. Lin, and U. Mohideen, 1999, Phys. Rev. D 60, 111101(R).

Rueckes, T., K. Kim, E. Joselevich, G. Y. Tseng, C. L. Cheung, and C. M. Lieber, 2000, Science 289, 94.

Rulis, P., L., Liang, and W. Y. Ching, 2009, unpublished.

Rydberg, H., B. I. Lundqvist, D. C. Langreth, and M. Dion, 2000, Phys. Rev. B 62, 6997.

Sabisky, E. S., and C. H. Anderson, 1973, Phys. Rev. A 7, 790. Saenger, W., 1984, Principles of Nucleic Acid Structure (Springer, New York).

Sahin, O., S. Magonov, C. Su, C. F. Quate, and O. Solgaard, 
2007, Nat. Nanotechnol. 2, 507.

Saint-Lager, M.-C., et al., 2007, Rev. Sci. Instrum. 78, 109902.

Saito, R., G. Dresselhaus, and M. S. Dresselhaus, 1999, Physical Properties of Carbon Nanotubes (Imperial College, London).

Schiros, T., L.-A. Naeslund, K. Andersson, J. Gyllenpalm, G. S. Karlberg, M. Odelius, H. Ogasawara, L. Pettersson, and A. Nilsson, 2007, J. Phys. Chem. C 111, 15003.

Schlesener, F., A. Hanke, R. Klimpel, and S. Dietrich, 2001, Phys. Rev. E 63, 041803.

Schoen, M., and S. H. L. Klapp, 2007, Eds., Nanoconfined Fluids: Soft Matter Between Two and Three Dimensions, Review in Comp. Chem. Vol. 24 (Wiley, Hoboken).

Schwegler, E., 2007, J. Phys.: Conf. Ser. 78, 012065.

Schwinger, J., 2004, Lett. Math. Phys. 88, 43.

Sedmik, R., I. Vasiljevich, and M. Tajmar, 2007, J. Comput.Aided Mater. Des. 14, 119.

Serry, F. M., D. Walliser, and G. J. Maclay, 1998, J. Appl. Phys. 84, 2501.

Shikler, R., T. Meoded, N. Fried, and Y. Rosenwaks, 1999 , Appl. Phys. Lett. 74, 2972.

Smay, J. E., J. Cesarano, and J. A. Lewis, 2002, Langmuir 18, 5429.

Sousa, J. F., P. A. Fernandez, and M. J. Ramos, 2007, J. Phys. Chem. A 111, 10439.

Sowerby, S. J., C. A. Cohn, W. M. Heckl, and N. G. Holm, 2001, Proc. Natl. Acad. Sci. U.S.A. 98, 820.

Sowerby, S. J., C. M. Morth, and N. G. Holm, 2001, Astrobiology 1, 481.

Soyka, F., O. Zvyagolskaya, C. Hertlein, L. Helden, and C. Bechinger, 2008, Phys. Rev. Lett. 101, 208301.

Sposito, G., 1998, Environ. Sci. Technol. 32, 2815.

Stack, A. G., S. R. Higgins, and C. M. Eggleston, 2001, Geochim. Cosmochim. Acta 65, 3055.

Staii, C., A. T. Johnson, M. Chen, and A. Gelperin, 2005, Arch. Hist. Exact Sci. 5, 1774.

Staroverov, V. N., G. E. Scuseria, J. Tao, and J. P. Perdew, 2003, J. Chem. Phys. 119, 12129.

Staroverov, V. N., G. E. Scuseria, J. Tao, and J. P. Perdew, 2004, Phys. Rev. B 69, 075102.

Stranski, I. N., 1942, Z. Phys. Chem. Abt. B 28, 425.

Strey, H. H., V. A. Parsegian, and R. Podgornik, 1999, Phys. Rev. E 59, 999.

Strey, H. H., R. Podgornik, D. C. Rau, and V. A. Parsegian, 1998, Curr. Opin. Struct. Biol. 8, 309.

Stumm, W., 1992, Chemistry of the Solid-Water Interface (Wiley, New York).

Sun, Y., B. Mayers, and Y. Xia, 2003, Arch. Hist. Exact Sci. 3, 675.

Sun, Y., and Y. Xia, 2002, Science 298, 2176.

Sutton, S. R., P. M. Bertsch, M. Newville, M. Rivers, A. Lanzirotti, and P. Eng, 2002, in Reviews in Mineralogy and Geochemistry 49, edited by P. A. Fenter, M. L. Rivers, N. C. Sturchio, and S. R. Sutton (Mineralogical Society of America, Washington, D.C.), p. 429.

Suzuki, A., E. Ohtani, T. Kondo, T. Kuribayashi, N. Niimura, K. Kurihara, and T. Chatake, 2001, Geophys. Res. Lett. 28, 3987.

Sverjensky, D. A., 2001, Geochim. Cosmochim. Acta 65, 3643. Sverjensky, D. A., 2003, Geochim. Cosmochim. Acta 67, 17.

Sverjensky, D. A., 2006, Geochim. Cosmochim. Acta 70, 2427.

Takahara, S., M. Nakano, S. Kittaka, Y. Kuroda, T. Mori, H. Hamano, and T. Yamaguchi, 1999, J. Phys. Chem. B 103,
5689.

Takahara, S., N. Sumiyama, S. Kittaka, T. Yamaguchi, and M.-C. Bellissent-Funel, 2005, J. Phys. Chem. B 109, 11231.

Takamuku, T., M. Yamagami, H. Wakita, Y. Masuda, and T. Yamaguchi, 1997, J. Phys. Chem. B 101, 5730.

Tamayo, J., and R. Garcia, 1998, Appl. Phys. Lett. 73, 2926.

Tan, G. L., M. F. Lemon, D. J. Jones, and R. H. French, 2005, Phys. Rev. B 72, 205117.

Tang, M., A. Ramos, E. Jud, S. Y. Chung, M. Gautier-Soyer, R. M. Cannon, W. C. Carter, and Y. M. Chiang, 2008, Langmuir 24, 1891.

Tang, M., et al., 2006, Phys. Rev. Lett. 97, 075502.

Tans, S. J., R. M. Verschueren, and C. Dekker, 1998, Nature (London) 393, 49.

Tapavicza, E., I.-C. Lin, O. A. von Lilienfeld, I. Tavernelli, M. Coutinho-Neto, and U. Rothlisberger, 2007, J. Chem. Theory Comput. 3, 1673.

Tasinkevych, M., and S. Dietrich, 2006, Phys. Rev. Lett. 97, 106102.

Tasinkevych, M., and S. Dietrich, 2007, Eur. Phys. J. E 23, 117. Tavares, F. W., D. Bratko, H. W. Blanch, and J. M. Prausnitz, 2004, J. Phys. Chem. B 108, 9228.

Teixeira, J., M.-C. Bellissent-Funel, S. H. Chen, and A. J. Dianoux, 1985, Phys. Rev. A 31, 1913.

Testard, F., L. Berthon, and T. Zemb, 2007, C. R. Chim. 10, 1034.

Thonhauser, T., V. R. Cooper, S. Li, A. Puzder, P. Hyldgaard, and D. C. Langreth, 2007, Phys. Rev. B 76, 125112.

Tkatchenko, A., and M. Scheffler, 2009, Phys. Rev. Lett. 102, 073005.

Tkatchenko, A., and O. A. von Lilienfeld, 2006, Phys. Rev. B 73, 153406.

Tkatchenko, A., and O. A. von Lilienfeld, 2008, Phys. Rev. B 78, 045116.

Todd, B., V. A. Parsegian, A. Shirahata, T. Thomas, and D. Rau, 2008, Biophys. J. 94, 4775.

Todd, B., and D. Rau, 2008, Nucleic Acids Res. 36, 501.

Todd, B., C. Stanley, N. Y. Sidorova, and D. C. Rau, 2007, Hydration Forces: Water and Biomolecules, Wiley Encyclopedia of Chemical Biology (Wiley, New York).

Torrie, G. M., and J. P. Valleau, 1982, J. Phys. Chem. 86, 3251. Tóth, G., 2007, J. Phys.: Condens. Matter 19, 335222.

Triantafillou, M., and R. D. Kamien, 1999, Phys. Rev. E 59, 5621.

Trizac, E., L. Bocquet, R. Agra, J.-J. Weis, and M. Aubouy, 2002, J. Phys.: Condens. Matter 14, 9339.

Tu, X., S. Manohar, A. Jagota, and M. Zheng, 2009, Nature (London) 460, 250.

van Blaaderen, A., 2006, Nature (London) 439, 545.

Van Oss, C. J., M. K. Chaudhury, and R. J. Good, 1987, Adv. Colloid Interface Sci. 28, 35.

Van Oss, C. J., M. K. Chaudhury, and R. J. Good, 1988, Chem. Rev. (Washington, D.C.) 88, 927.

Veble, G., and R. Podgornik, 2007a, Eur. Phys. J. E 23, 275.

Veble, G., and R. Podgornik, 2007b, Phys. Rev. B 75, 155102.

Verma, R., J. C. Crocker, T. C. Lubensky, and A. G. Yodh, 1998, Phys. Rev. Lett. 81, 4004.

Verwey, E. J. W., and J. Th. G. Overbeek, 1948, Theory of the Stability of Lyophobic Colloids (Elsevier, Amsterdam).

Vogel, S. R., M. M. Kappes, F. Hennrich, and C. Richert, 2007, Chem.-Eur. J. 13, 1815.

von Lilienfeld, O. A., and D. Andrienko, 2006, J. Chem. Phys. 124, 054307. 
von Lilienfeld, O. A., R. Lins, and U. Rothlisberger, 2005, Phys. Rev. Lett. 95, 153002.

von Lilienfeld, O. A., I. Tavernelli, U. Rothlisberger, and D. Sebastiani, 2004, Phys. Rev. Lett. 93, 153004.

von Lilienfeld, O. A., I. Tavernelli, U. Rothlisberger, and D. Sebastiani, 2005, Phys. Rev. B 71, 195119.

Wada, S.-I., M. Takigawa, K. Matsushita, H. Kizaki, and K. Tanaka, 2007, Surf. Sci. 601, 3833.

Wang, H., D. W. Brandl, F. Le, P. Norlander, and N. J. Halas, 2006, Arch. Hist. Exact Sci. 6, 827.

Wang, Y., T. Uchida, F. Westferro, M. L. Rivers, N. Nishiyama, J. Gebhardt, C. E. Lesher, and S. R. Sutton, 2005, Rev. Sci. Instrum. 76, 073709/1.

Waterman, P. C., 1971, Phys. Rev. D 3, 825.

Waychunas, G., 2002, in Reviews in Mineralogy and Geochemistry, edited by P. A. Fenter, M. L. Rivers, N. C. Sturchio, and S. R. Sutton (Mineralogical Society of America, Washington, D.C.), Vol. 49, p. 267.

Waychunas, G., T. Trainor, P. Eng, J. Catalano, G. Brown, J. Davis, J. Rogers, and J. Bargar, 2005, Anal. Bioanal. Chem. 383, 12.

Weisman, R. B., 2003, Nature Mater. 2, 569.

Wenk, H.-R., 2006, Ed., Reviews in Mineralogy and Geochemistry Neutron, Scattering in Earth Science Vol. 63 (Mineralogical Society of America, Washington, D.C.).

Wennerström, H., A. Khan, and B. Lindman, 1991, Adv. Colloid Interface Sci. 34, 433.

Wernersson, E., and R. Kjellander, 2007, J. Phys. Chem. B 111, 14279.

Wesolowski, D. J., M. L. Machesky, D. A. Palmer, and L. M. Anovitz, 2000, Chem. Geol. 167, 193.

Wesolowski, D., M. Machesky, M. Ridley, D. Palmer, Z. Zhang, P. Fenter, M. Predota, and P. Cummings, 2008, ECS Trans. 11, 27.

Wettlaufer, J. S., 1999, Phys. Rev. Lett. 82, 2516.

Wettlaufer, J. S., and M. G. Worster, 2006, Annu. Rev. Fluid Mech. 38, 427.

Whitesides, G. M., and M. Boncheva, 2002, Proc. Natl. Acad. Sci. U.S.A. 99, 4769.
Whitesides, G. M., and B. Grzybowski, 2002, Science 295, 2418.

Wilen, L. A., et al., 1995, Phys. Rev. B 52, 12426.

Williams, H. J., and C. F. Chabalowski, 2001, J. Phys. Chem. A 105, 646.

Wind, S. J., J. Appenzeller, and P. Avouris, 2003, Phys. Rev. Lett. 91, 058301.

Wu, Q., and W. Yang, 2002, J. Chem. Phys. 116, 515.

Wu, X., M. C. Vargas, S. Nayak, V. Lotrich, and G. Scoles, 2001, J. Chem. Phys. 115, 8748.

Xu, X., and W. A. Goddard, 2004, Proc. Natl. Acad. Sci. U.S.A. 101, 2673.

Yeh, I.-C., and M. L. Berkowitz, 1999, J. Chem. Phys. 110, 7935.

Zandi, R., J. Rudnick, and M. Kardar, 2004, Phys. Rev. Lett. 93, 155302.

Zanotti, J.-M., M.-C. Bellissent-Funel, and S. H. Chen, 1999, Phys. Rev. E 59, 3084.

Zhang, Y., and W. Yang, 1998, Phys. Rev. Lett. 80, 890.

Zhang, Z., P. Fenter, N. C. Sturchio, M. J. Bedzyk, M. L. Machesky, and D. J. Wesolowski, 2007, Surf. Sci. 601, 1129.

Zhang, Z., and S. C. Glotzer, 2004, Nano Lett. 4, 1407.

Zhang, Z., et al., 2004, Langmuir 20, 4954.

Zhao, Y., and D. G. Truhlar, 2005a, J. Chem. Theory Comput. 1, 415.

Zhao, Y., and D. G. Truhlar, 2005b, J. Phys. Chem. A 109, 5656.

Zheng, M., A. Jagota, E. Semke, B. Diner, R. Mclean, S. Lustig, R. Richardson, and N. Tassi, 2003, Nature Mater. 2, 338.

Zheng, M., A. Jagota, M. S. Strano, A. P. Santos, P. Barone, S. G. Chou, B. A. Diner, M. S. Dresselhaus, R. S. Mclean, G. B. Onoa, G. G. Samsonidze, E. D. Semke, M. Usrey, and D. J. Walls, 2003, Science 302, 1545.

Zheng, M., and E. D. Semke, 2007, J. Am. Chem. Soc. 129, 6084.

Zhou, X. Y., X. J. Wei, M. V. Fedkin, K. H. Strass, and S. N. Lvov, 2003, Rev. Sci. Instrum. 74, 2501.

Zhu, Y., and S. Granick, 2001, Phys. Rev. Lett. 87, 096104. 